\title{
Transition of intrinsically unfolded $\alpha$-synuclein into \\ the fibrillar state characterized by NMR spectroscopy
}

\author{
Dissertation \\ zur Erlangung des Doktorgrades \\ der Mathematisch-Naturwissenschaftlichen Fakultäten \\ der Georg-August-Universität zu Göttingen
}

vorgelegt von

Min-Kyu Cho

aus Seoul, Süd Korea

Göttingen 2008 
D7

Referent: Prof. Dr. Christian Griesinger

Korreferent: Prof. Dr. Bernd Abel

Tag der mündlichen Prüfung: 
세상 속 나의 모든 가족들에게 



\section{Publication list}

This thesis is based on the following publications:

1. Min-Kyu Cho, Hai-Young Kim, Pau Bernado, Claudio O. Fernandez, Martin Blackledge and Markus Zweckstetter: Amino Acid Bulkiness Defines the Local Conformations and Dynamics of Natively Unfolded $\alpha$-Synuclein and Tau. J. Am. Chem. Soc. 2007: 129(11): 3032-3.

2. Min-Kyu Cho, Hai-Young Kim, Claudio O. Fernandez, and Markus Zweckstetter: Secondary structure arrangements in amyloid fibrils of wildtype and A30P a-synuclein. (In preparation)

3. Hai-Young Kim, Min-Kyu Cho, Dietmar Riedel, Claudio O. Fernandez and Markus Zweckstetter: Cold denaturation of amyloid fibrils of alpha-synuclein in supercooled water. Angew. Chem. Int. Ed. Engl. 2008: 47(27): 5046-8.

4. Hai-Young Kim, Min-Kvu Cho, Ashutosh Kumar, Dietmar Riedel, Roland Benz, Claudio O. Fernandez, Hilal A. Lashuel, Sebastian Kügler, Adam Lange, Christian Griesinger and Markus Zweckstetter: High-resolution characterization of the structure of toxic a -synuclein oligomers. (in preparation)

Other publications:

5. Lukasz Skora, Min-Kyu Cho, Hai-Young Kim, Stefan Becker, Claudio O. Fernandez, Martin Blackledge and Markus Zweckstetter: Charge-induced molecular alignment of intrinsically disordered proteins. Angew. Chem. Int. Ed. Engl. 2006: 45(42): 7012-5. 
6. Ralf Jauch, Min-Kyu Cho, Stefan Jäkel, Catharina Netter, Kay Schreiter, Babette Aicher, Markus Zweckstetter, Herbert Jäckle and Markus C. Wahl: Mitogen-activated protein kinases interacting kinases are autoinhibited by a reprogrammed activation segment. EMBO J. 2006: 25(17): 4020-32. 


\section{Acknowledgement}

This research work was carried at the department of NMR based Structural Biology, Max Planck Institute for Biophysical Chemistry, Göttingen, Germany, under supervision of Prof. Dr. Christian Griesinger and Prof. Dr. Markus Zweckstetter.

First of all, I would like to express my gratitude to the director of the department, Prof. Dr. Christian Griesinger, and my direct supervisor, Prof. Dr. Markus Zweckstetter for providing me guidance, encouragement, and a great opportunity to achieve my PhD with the exciting project at MPI that I would say one of the world best places for researchers.

I would like thank Prof. Dr. Bernd Abel for being my 'Korreferent' at Faculty of Chemistry, Georg-August University Göttingen, and Prof. Dr. George Sheldrick, Prof. Dr. J oerg Schroeder, Prof. Dr. Michael Buback, Prof. Dr. Franc Meyer for accepting to be a member of my external PhD committee, and Prof. Dr. Martin Suhm for the financial and scientific support through Physical and Chemical Graduate School Göttingen (GRK 782) for the first three years of my PhD.

Collaborations with great colleagues have been fundamental in the project, and some of them are presented on this thesis. I'm indebt to Hai-Young Kim, D. Pinar Karpinar, Lukasz Skora, Dr. Thomas Jovin, Dr. Claudio 0. Fernandez, Dr. Carlos Bertoncini for all my research on $\alpha$-synuclein, Dr. Martin Blackledge, Dr. Pau Bernadó, Dr. Malene Ringkjøbing Jensen for their excellent computational modeling and collaboration on RDC of $\alpha$ synuclein, Dr. Dietmar Riedel for his patience to make EM images of a 
number of 'tricky' samples, Prof. Dr. Hilal Lashuel, Asad J an for their help in $\alpha$-synuclein oligomer imaging, Prof. Dr. Marc Baldus, Prof. Dr. Hendrike Heise, Dr. Ashtosh Kumar for their professional work with solid-state NMR, Dr. Stefan Becker, Karin Giller for all help in biological work during my PhD, Dr. Alexandra Klos Witkowoska, Gonzalo R. Lamberto for their help in analysis of NMR spectra, Gerhard Wolf for his help with mass spectroscopy and Dr. Markus Wahl, Dr. Ralf J auch for the collaboration on Mnk2.

Not only for my project, am I also grateful who shared all the exciting moment with NMR and computation. Dr. Vinesh Vijayan and ShengQi Xian helped me to translate all symbols into NMR experiments. Dr. J eganath Korukottu and Raghavendran Lakshmi Narayanan made my idea work in computer with their coding. Lukasz Skora, Dr. Christophe Fares, Dr. Ulrich Dürr shared lots of fun to make our NMRs work. I would thank my former and present lunch members, Vinesh, Fernando, ShengQi, Laura, Ulrich, Sebastian, Mitch, Michael, Pierre, Stefan, Nicolas, Dr. Donghan Lee who share one of the best time in every working day and even after.

I also shared my work and joy with all colleagues in NMR department, J ulian, Monika, Saskia, Dirk, Edward, Hessam, Stefen, Korvin, Marco, Jörg, Mate, Nils, Melanie, Uwe, Valerie, Francesca, Christian, Robert, Armando, Anni, Sergey, Fabian, Edith, Holger, Marcel and Mrs. Silberer.

I would mention my friends in MPIbpc, Shamala, Soleda, Fito, Jürgen, Matias, not only for the scientific discussion, but also for friendly conversation that watered the dry work life.

Prof. Dr. Wontae Lee, Prof. Dr. Yu Sam Kim should have my gratitude for their instruction and supports for my scientific life. 
My appreciation should be given to Pastor Jae-J ung Ahn, all friends in Korean Church in Göttingen, for their religious life in Göttingen, and Dr. J ung-Sup Rhee, Sunhae Park for their advices and for their sharing of social life in Göttingen.

I thank deeply Hyewoong Kim, Kyu-Young Han, Yong Shin, Eun-J ung Kim for their warmth given to me and for sharing their joyful time with me from the beginning of my PhD..

I am deeply grateful Hai-Young Kim, for her love and ceaseless support for my work and my life from the master till now.

Last, I appreciate My parents, and my brother, J un-Kyu for their endless love and being with me from my birth, and All my grandparents and family members in the world who have been great supporters from the beginning of my life. 



\section{Abstract}

Proteins fold into appropriate configuration, called native structure, in order to achieve its cellular function. A protein with nonnative structure induces malfunction and causes the relevant disease. Such protein misfolding has been revealed as a common pathogenic process in many neurodegenerative diseases like Alzheimer's and Parkinson's disease. In Parkinson's disease (PD), a protein called $\alpha$-synuclein $(\alpha \mathrm{S})$ is found a major component of Lewy body, a proteinaceous aggregate with amyloid fibril form of $\alpha \mathrm{S}$. Considering its character as an intrinsically unfolded protein, the overall change of conformation during PD is quite attractive for biophysicist to understand protein folding and misfolding, and the detailed information can lead to a therapeutic achievement for the treatment of PD.

In this thesis, I have investigated the transition of an intrinsically unfolded $\alpha \mathrm{S}$ into amyloid fibril with NMR spectroscopy and various biophysical methods.

Natively unfolded proteins play key roles in normal and pathological biochemical processes. When confined in weakly aligning media, natively unfolded proteins such as $\alpha \mathrm{S}$ display surprisingly variable NMR dipolar couplings as a function of position along the chain, suggesting the presence of residual secondary or tertiary structure. In Chapter 3, it is shown that that the variation of NMR dipolar couplings and heteronuclear relaxation rates in $\alpha \mathrm{S}$ closely follows the variations of the bulkiness of amino acids along the polypeptide chain. The results demonstrate that the bulkiness of amino acids defines the local conformations and dynamics of $\alpha \mathrm{S}$ and other natively 
unfolded proteins. Deviations from this random coil behavior can provide insight into residual secondary structure and long-range transient interactions in unfolded proteins.

The transition from natively unfold conformation into amyloid fibril starts with a change in monomeric conformation. Previously it has been shown that $\alpha \mathrm{S}$ adopts an autoinhibitory conformation in physiological condition. Changes in environmental factors like low $\mathrm{pH}$, molecular crowding agents, high temperature, and/or high salt concentration accelerate $\alpha \mathrm{S}$ aggregation. In these conditions, $\alpha \mathrm{S}$ may transform into an aggregation-prone, partially folded intermediate, and such dimensional change at $\mathrm{pH} 3$ was observed with CD, SAXS and fluorescence. As described in Chapter 4, NMR spectroscopy was applied to address such conformational change in atomic resolution.

Chapter 5 describes the distinction between wt and A30P, a genetic mutant of $\alpha \mathrm{S}$, amyloid fibril core. Here we took the advantage of HR-MAS NMR spectroscopy to detect flexible regions in amyloid fibrils. Together with hydrogen/deuterium (H/D) exchange experiments, the arrangement of $\beta$-strands and loops in fibrillar core region is shown. Longer amyloid fibril core region for A30P is observed compared to wt amyloid fibril; the reason for the difference, however, should be addressed.

Chapter 6 consists of a brief description about the conformation of the $\alpha \mathrm{S}$ oligomer derived from amyloid fibril in supercooled aqueous solution. Electron microscopy (EM) and atomic force microscopy (AFM) reveals spherical conformation with variation in diameter and height. This study, combined with physiological investigation, would lead better understanding of the intermediates in amyloid fibril formation. 


\section{Table of Contents}

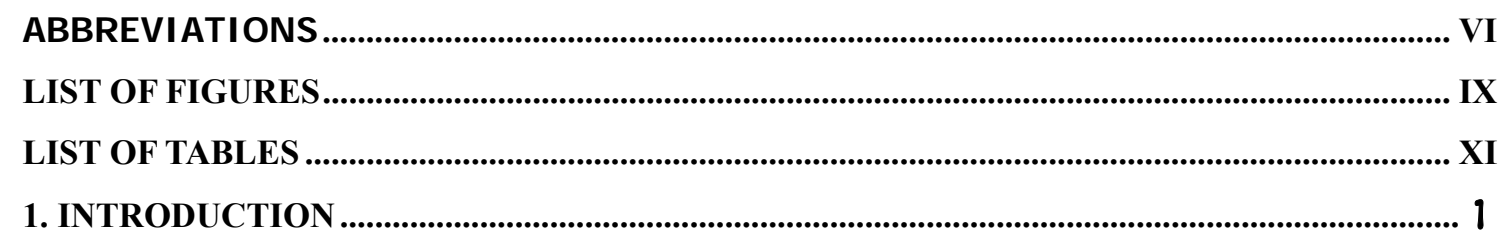

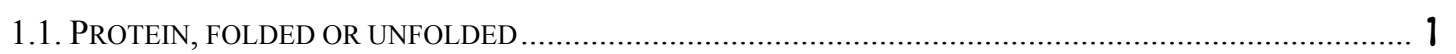

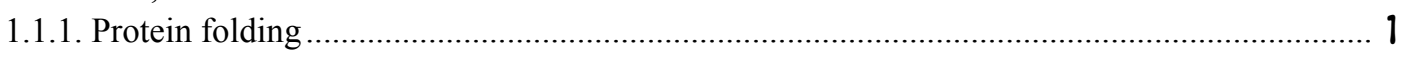

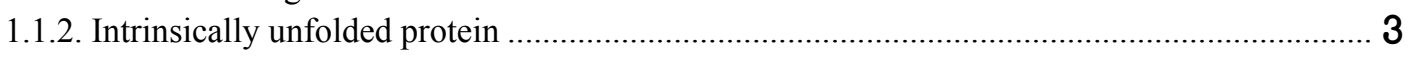

1.1.2.1. Conformational restrictions in unfolded proteins ........................................................................ 3

1.1.2.2. In vitro methods for the characterization of unfolded protein ......................................................... 4

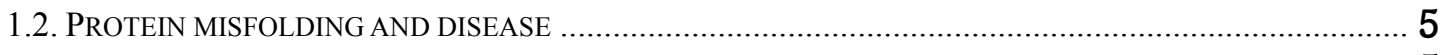

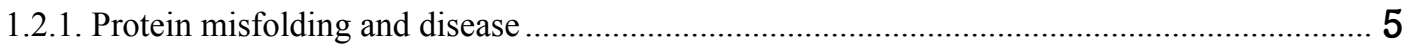

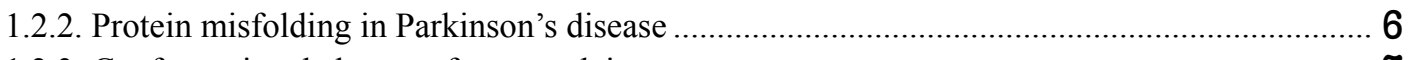

1.2.3. Conformational change of $\alpha$-synuclein ........................................................................ 7

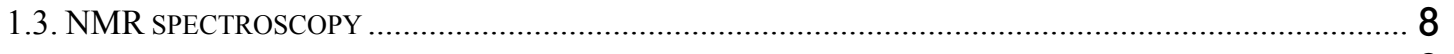

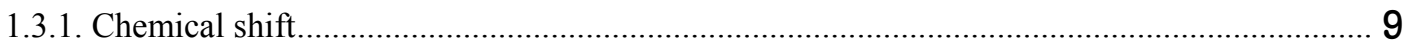

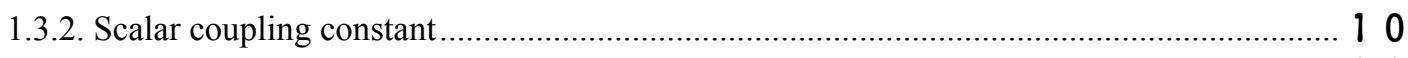

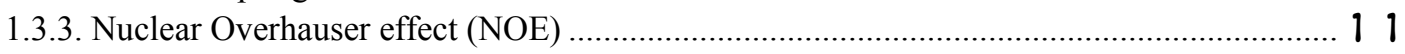

1.3.4. Paramagnetic relaxation enhancement (PRE) .................................................................. 1

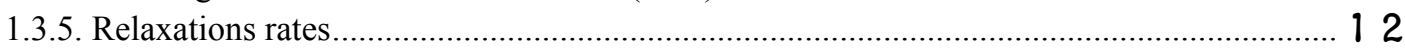

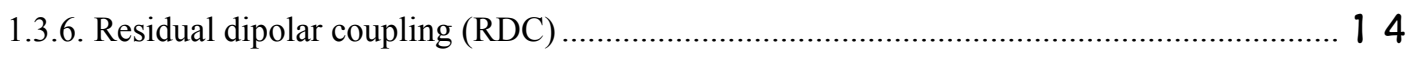

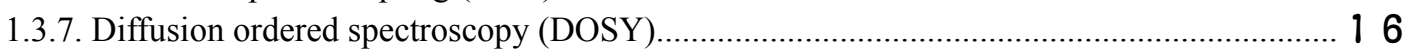

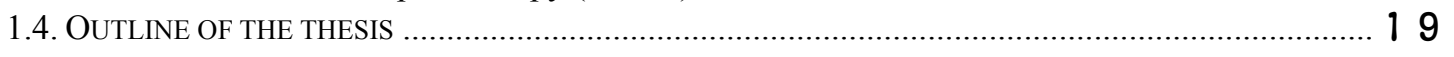

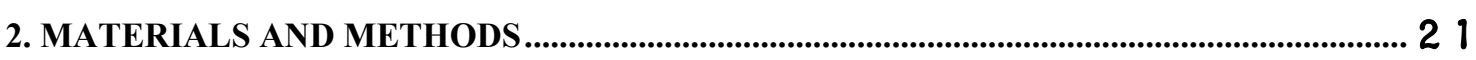

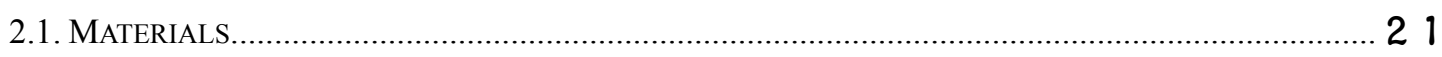

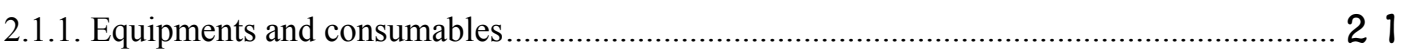

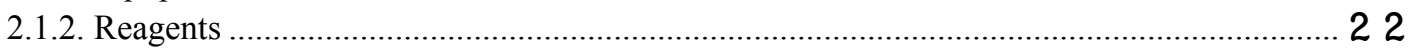

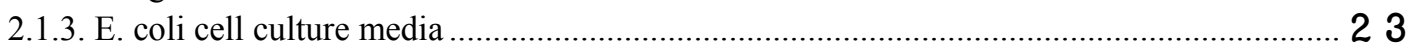

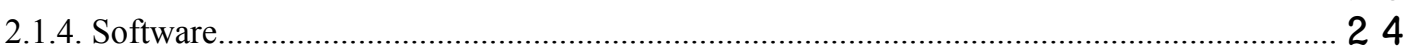

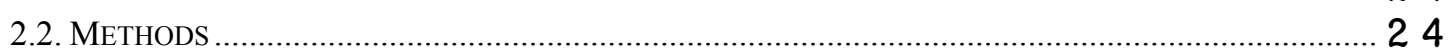

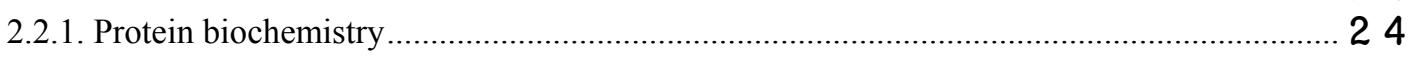

2.2.1.1. Protein expression and cultivation of E. coli......................................................................... 24

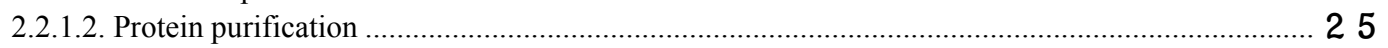

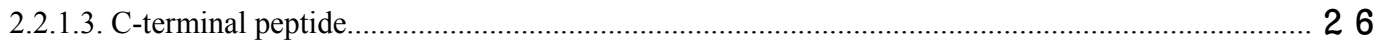

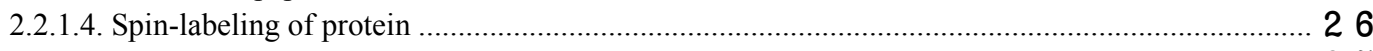

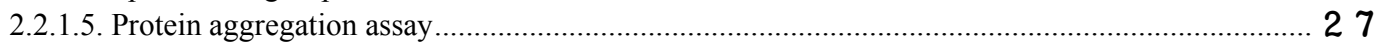

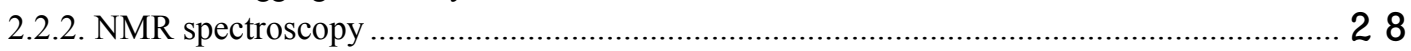

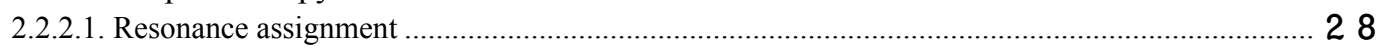

2.2.2.2. Secondary structure determination ............................................................................. 30

2.2.2.3. Chemical shift difference ......................................................................................... 30

2.2.2.4. Diffusion coefficient and hydrodynamic radius ......................................................................... 30 


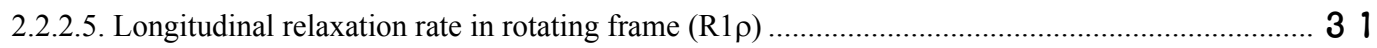

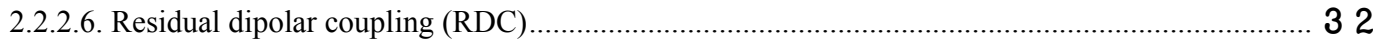

2.2.3. Other spectroscopic methods ............................................................................... 33

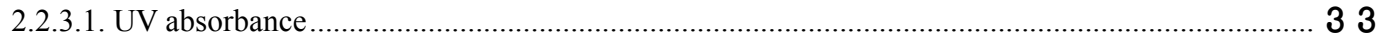

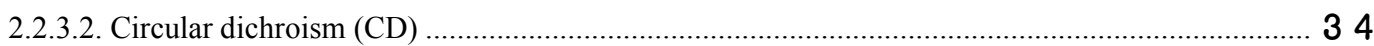

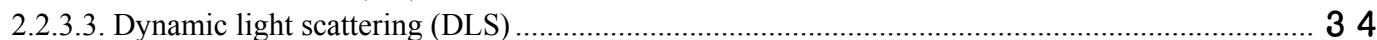

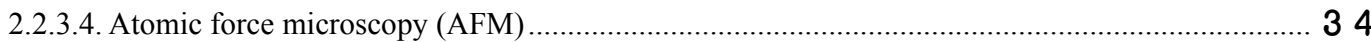

3. AMINO ACID BULKINESS DEFINES THE LOCAL CONFORMATIONS AND DYNAMICS

OF NATIVELY UNFOLDED $\alpha$-SYNUCLEIN ....................................................................... 35

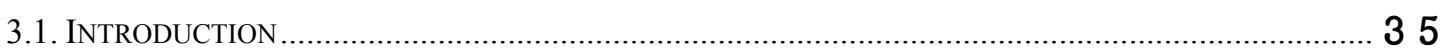

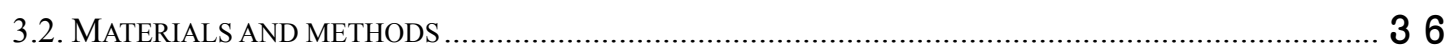

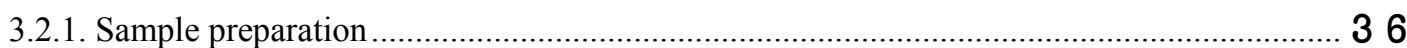

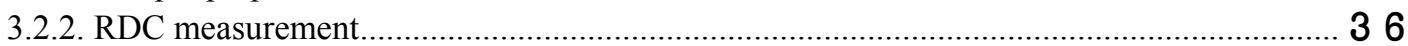

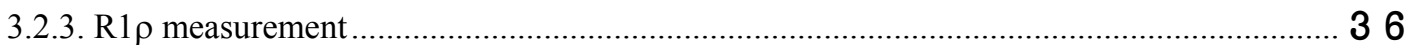

3.2.4. Calculation of bulkiness profile...................................................................................... 37

3.2.5. Flexible-meccano model generation and RDC calculation................................................... 37

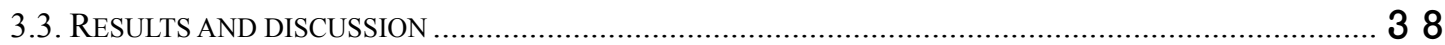

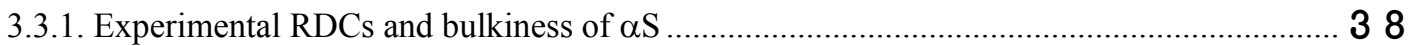

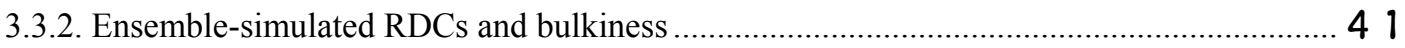

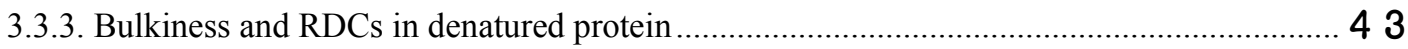

3.3.4. Comparison of bulkiness with other physico-chemical properties ...................................... 46

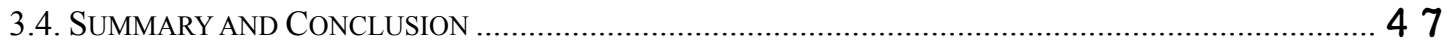

4. AGGREGATION PRONE CONFORMATION OF $\alpha$-SYNUCLEIN AT LOW PH.................. 49

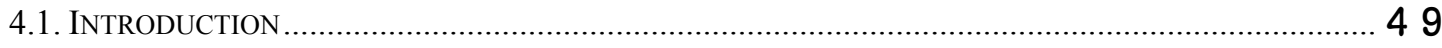

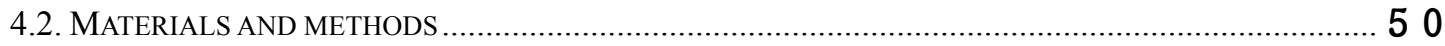

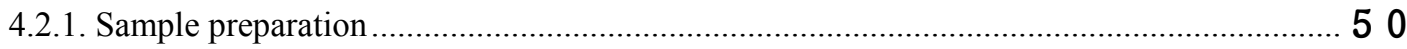

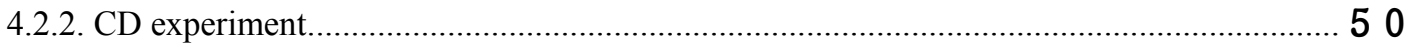

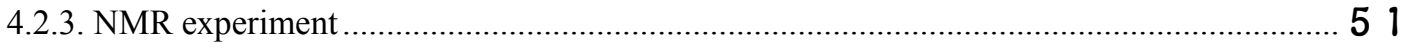

4.3. RESULTS AND DISCUSSION............................................................................................ 5

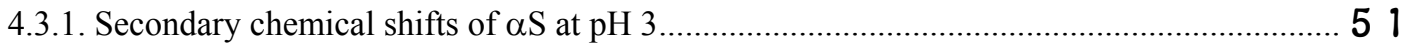

4.3.2. PRE and hydrodynamic radius revealed collapsed C-terminus ........................................... 54

4.3.3. Changes in protein dynamics and structure detected by RDCs .......................................... 57

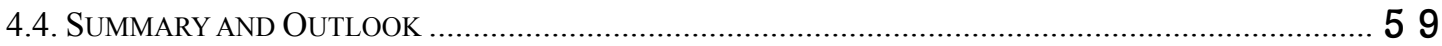

\section{CROSS- $\beta$ SHEET CORE OF $\alpha$-SYNUCLEIN AMYLOID FIBRIL DETERMINED BY}

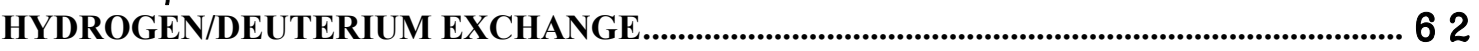

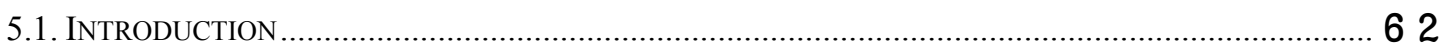

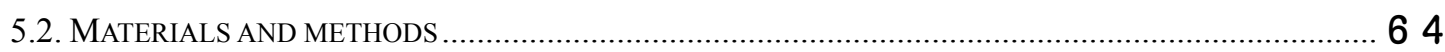

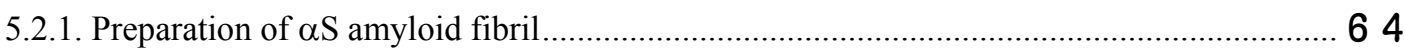

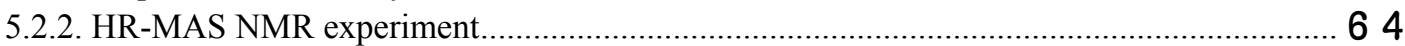

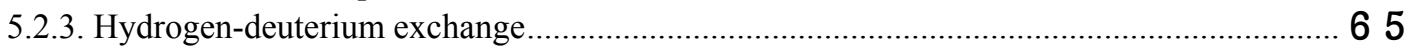

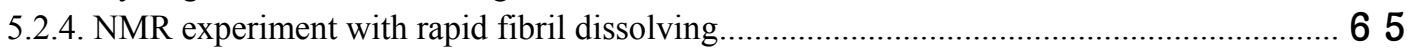

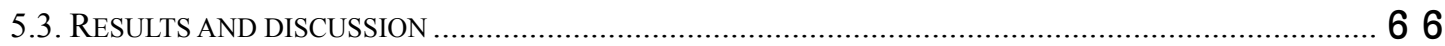

5.3.1. Flexible regions in $\alpha \mathrm{S}$ amyloid fibril detected by HR-MAS ............................................ 66

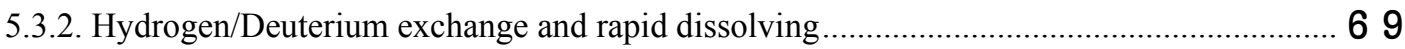

5.3.3. Intensity profiles of 1 week H/D exchanged fibrils ......................................................... 71

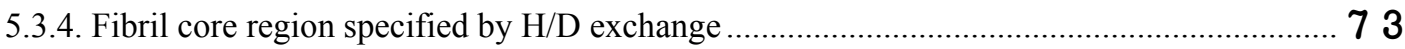

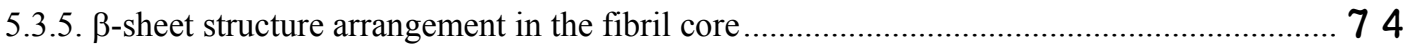

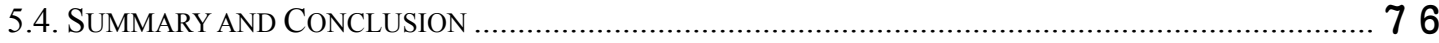


6. CONFORMATION STUDY ON $\alpha$-SYNUCLEIN OLIGOMERS DERIVED FROM AMYLOID FIBRILS IN SUPERCOOLED SOLUTION

6.1. INTRODUCTION

78

6.2. MORPHOLOGY OF THE OLIGOMERS

78

6.3. SUMMARY AND OUTLOOK

7. BIBLIOGRAPHY

82

APPENDIX

94

A.1. WT $\alpha$ S BACKBONE CHEMICAL SHIFTS AT PH3 IN NA-ACETATE ...

94 


\section{Abbreviations}

$\mathbf{A} \beta$

AD

APP

$\alpha \mathbf{S}$

$\alpha S(1-108)$

ATP

BO

$\beta \mathbf{S}$

CD

CSA

DNA

${ }^{1} D_{\mathrm{NH}}$

DSSE

DTT

E. coli

EDTA

EM

EPR

ER

FRET

FT

$\gamma \mathbf{S}$

HEPES

HSQC

HSPs

I NEPT
Amyloid- $\beta$ peptide

Alzheimer's disease

Amyloid precursor protein

$\alpha$-synuclein

C-terminal truncated $\alpha$-synuclein (residue 1-108)

Adenosine -5 '-Triphosphate

External magnetic field

$\beta$-synuclein

Circular dichroism

Chemical shift anisotropy

Deoxyribonucleic acid

$\mathrm{N}-\mathrm{H}$ residual dipolar coupling

Doublet Separated Sensitivity Enhanced

Dithiothreitol

Escherichia coli

Ethylenediamine tetraacetic acid

Electron microscopy

Electron paramagnetic resonance

Endoplasmic reticulum

Förster resonance energy transfer

Fourier-transform

$\gamma$-synuclein

2-[4-(2-hydroxyethyl)1-1 piperazinyl] ethansulfonic acid

Heteronuclear single quantum coherence

Heat shock proteins

Insensitive nuclei enhanced by polarization transfer 
I PAP

I PTG

I UP

${ }^{1} \mathrm{~J}_{\mathrm{NH}}$

${ }^{3}$ HNH $\alpha$

LBs

$\mathbf{m L}$

ms

$\mu \mathrm{L}$

$\mu \mathbf{S}$

MTSL

MWCO

NAC

NMR

NOE

ns

OD

PD

pD

PFG

PG-SLED

PMSF

PI I

PRE

PrP

ps

RDC
In-Phase-Anti-Phase

Isopropyl- $\beta$-D-thiogalactopyranoside

Intrinsically unfolded (unstructured) protein

One bond $\mathrm{N}-\mathrm{H}$ coupling

Three bond $\mathrm{H}_{\mathrm{N}}-\mathrm{H}_{\alpha}$ coupling

Lewy bodies

Milliliter

Milliseconds

Micro liter

Microseconds

(1-oxy-2,2,5,5-tetramethyl-D-pyrroline-3-methyl)-

methanethiosulfonate

molecular weight cutoff

Non-A $\beta$ Component of Alzheimer's disease amyloid plaques

Nuclear magnetic resonance

Nuclear overhausser effect

Nanoseconds

Optical density

Parkinson's disease

$\mathrm{pH}$ without isotope effect correction

Pulse field gradient

Pulse gradient stimulated echo longitudinal encode-decode Phenylmethylsulfonyl fluoride

Polyproline II

Paramagnetic relaxation enhancement

Prion protein

Picoseconds

Residual dipolar coupling 
$\mathbf{R}_{\mathbf{1}_{\rho}} \quad$ Longitudinal relaxation rate in rotating frame

$\mathbf{R}_{\mathbf{g}} \quad$ Radius of gyration

$\mathbf{R}_{\mathbf{h}} \quad$ Hydrodynamic radius

SAXS Small angle X-ray scattering

SDS Sodium dodecyl sulphate

SDS-PAGE SDS-polyacrylamide gel electrophoresis

Thiot Thioflavin-T

UPS Ubiquitin-proteasomal system

wt Wild type 


\section{List of Figures}

Fig. 1- 1. Schematic energy landscapes for protein folding. .................................................. 2

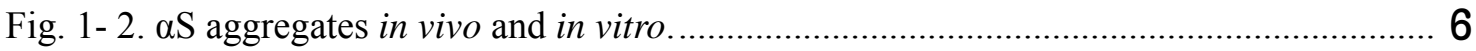

Fig. 1- 3. Schematic view of conformational change of $\alpha \mathrm{S}$. ................................................. 7

Fig. 1- 4. Dihedral angles in polypeptide chain and Ramachandran plot................................. 4

Fig. 1- 5. The time scale of protein dynamics and corresponding NMR parameters................. 9

Fig. 1- 6. One-bond scalar coupling constants in peptide. .................................................. 10

Fig. 1- 7. Schematic representation of the orientational dependence of RDC on the alignment tensor.

Fig. 1- 8. PG-SLED spectra of internal reference, dioxane, and $\alpha \mathrm{S}$. ................................ 17

Fig. 2- 1. Reaction of MTSL with Cys residue in a protein. ................................................... 27

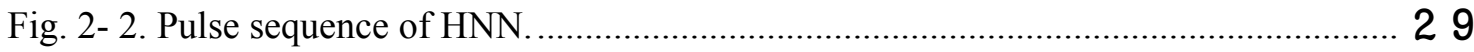

Fig. 2- 3. Pulse sequence of PG-SLED. ……....................................................................... 3

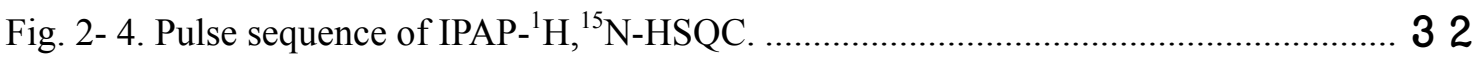

Fig. 2- 5. Pulse sequence of IPAP-(HA)CANH. ............................................................... 33

Fig. 3- 1. Bulkiness profile and NMR dynamics parameters of $\alpha \mathrm{S}$..................................... 39

Fig. 3- 2. Comparison of the raw bulkiness profile with one-bond RDCs of $\alpha \mathrm{S}$. ................... 41

Fig. 3- 3. Representative conformations generated by flexible-meccano. ............................ 42

Fig. 3- 4. Comparison of amino acid bulkiness with ensemble-averaged RDCs. ................... 42

Fig. 3- 5. Comparison of RDCs predicted with/ without steric obstruction........................... 43

Fig. 3- 6. Comparison of bulkiness and ${ }^{1} \mathrm{D}_{\mathrm{NH}}$ of unfolded proteins. .................................... 44

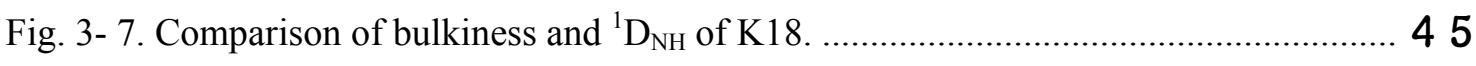

Fig. 3- 8. Comparison of residue specific physico-chemical properties. ................................ 46

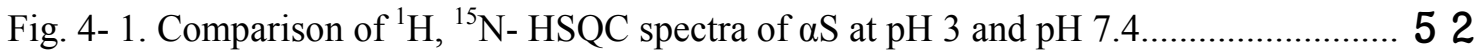

Fig. 4- 2. Secondary chemical shifts of $\alpha \mathrm{S}$ at $\mathrm{pH} 3$ compared with $\mathrm{pH} 7.4$ values................. 54

Fig. 4- 3. Comparison of PRE profiles at $\mathrm{pH} 3$ (blue bars) and at $\mathrm{pH} 7.4$ (magenta line)..... 55

Fig. 4- 4. CD spectra of full-length wt $\alpha \mathrm{S}$ (blue) and C-terminal peptide (cyan)................... 56 
Fig. 4- 5. Hydrodynamic radii of $\alpha \mathrm{S}(1-108)$ and $\mathrm{C}$-terminus at different $\mathrm{pH}$.

Fig. 4- 6. ${ }^{1} \mathrm{D}_{\mathrm{NH}}$ of full-length $\alpha \mathrm{S}$ and C-terminal peptide (105-136) at $\mathrm{pH} 7.4$ vs. at $\mathrm{pH} 3 \ldots . . .58$

Fig. 4- 7. Schematic model of $\alpha \mathrm{S}$ conformational change at $\mathrm{pH} 3$ 61

Fig. 5- 1. EM image and ThT florescence of $\alpha \mathrm{S}$ amyloid fibril. 66

Fig. 5- 2. Comparison of $2 \mathrm{D}{ }^{1} \mathrm{H}^{15} \mathrm{~N}$ HSQC spectra from $w t \alpha \mathrm{S}$ fibril and from monomer. ... 68 Fig. 5- 3. Residue specific peak intensity from $2 \mathrm{D}{ }^{1} \mathrm{H}_{-}{ }^{15} \mathrm{~N}$ HSQC spectra of $w t \alpha \mathrm{S}$ fibril in HRMAS. 69

Fig. 5- 4. Average peak intensity decay curves in 50\% D2O dissolving buffer. 70

Fig. 5- 5. Peak intensity modulations of one week exchanged fibrils 72

Fig. 5- 6. Back calculated peak intensity after 1 week H/D exchange. 73

Fig. 5- 7. Peak intensity profile in the fibril core region of $\alpha \mathrm{S}$. 75

Fig. 6- 1. The resolution of microscopic methods and the dimension of biomolecules and chemicals 79

Fig. 6- 2. EM image and Thioflavin-T fluorescence of the oligomers. 80

Fig. 6- 3. Atomic force microscopy image of the oligomers. 80 


\section{List of Tables}

Table 1- 1. Biophysical methods for the unfolded proteins..................................................... 4

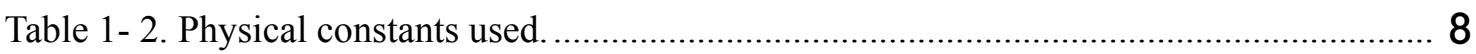

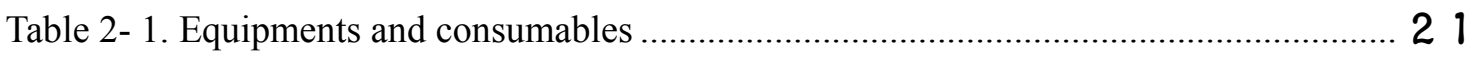

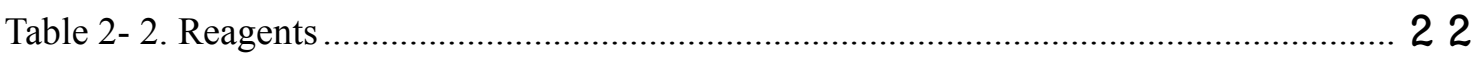

Table 2- 3. E.coli cell culture media contents .................................................................. 23

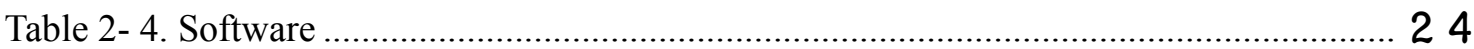

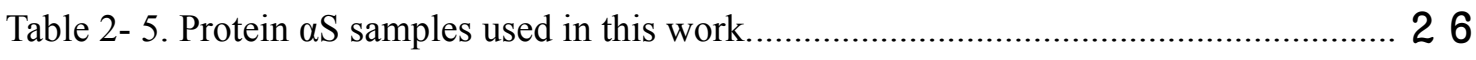





\section{Introduction}

\subsection{Protein, folded or unfolded}

\subsubsection{Protein folding}

Proteins, one of main constituents of an organism, are polymers comprising 20 chemically and structurally different building blocks, only L- $\alpha$-amino acids for naturally occurring proteins. Amino acid sequences in proteins are encoded in corresponding genes in form of deoxyribonucleic acid (DNA). Protein biosynthesis from a DNA sequence consists of two steps, transcription and translation. A gene encoded in a DNA sequence is transcribed into a messenger ribonucleic acid (mRNA), and then the mRNA is used as a template by ribosomal RNA to combine specific amino acids into a protein. After translation, a protein can undergo posttranslational modification like glycosylation, alkylation, phosphorylation and so forth (Berg et al. 2002).

The function of a protein is originated from its three-dimensional configuration, called tertiary structure. The process by which a linear polypeptide chain, called primary structure, spontaneously folds into its functional three-dimensional native conformation is entitled protein folding (McEntyre 2001, Dill et al. 2008). The first theoretical concept about the protein folding was suggested by Cyrus Levinthal in 1969 (Levinthal 1969, Baldwin 2008, Fersht 2008). In his concept, each amino acid is supposed to adopt one of three conformational states; $\alpha$-helix, $\beta$-strand, or random coil, randomly and independently. This process requires astronomical time for an unfolded protein to fold into a native structure (i.e. 100 residue polypeptide should search 3100 conformations) 
and is unable to explain the biological time scale of microseconds to milliseconds in spontaneous folding of an unfolded protein. In consequence, the random search process is entitled as Levinthal paradox, and from this very beginning of the theoretical study on protein folding, researchers have made great effort to answer three closely related questions: What is the folding code? What is the folding mechanism? and is it possible to predict the native structure of a protein from its amino acid sequence (Dill et al. 2008)?
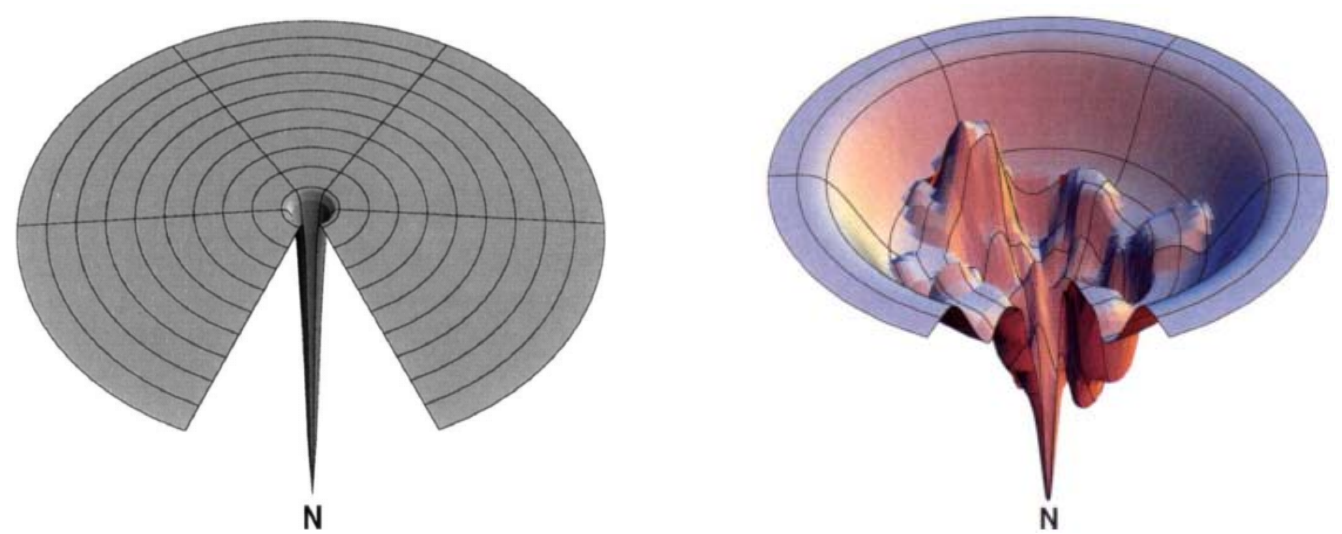

Fig. 1- 1. Schematic energy landscapes for protein folding.

$\mathrm{N}$ is the native conformation. Folding is done with random search for $\mathrm{N}$ in Levinthal landscape (left), while it is done with kinetic traps, energy barriers and some narrow throughway paths in a rugged energy landscape (right) (adapted and modified from Dill and Chan(Dill and Chan 1997)).

A part of answer for the folding code was achieved by Anfinsen who revealed that a protein itself is enough to make the protein fold into its native structure and the native structure should be at the global minimum in energy landscape (Anfinsen 1973). With the discovery, great efforts combined with many experimental methods on proteins have been developed to address the complete folding code and the related folding mechanism in a relationship with a designated energy landscape (Dill and Chan 1997, Dill et al. 
2008, Fersht 2008). Recent progress in the field shows proteins fold with a series of local optimizations that leads to the global optimization of the protein, and through multiple pathways depending on the folding conditions (Dill et al. 2008, Udgaonkar 2008). All those accumulated knowledge has led more accurate structure prediction of proteins up to 150 residues solely with amino acid sequence (Hardin et al. 2002, Zhang 2008).

\subsubsection{Intrinsically unfolded protein}

Since the first protein structure was determined, a well-defined protein structure was regarded as a necessity for protein function, and in accordance with higher functionality, higher organism was supposed to have more genes with folded proteins. To the contrary, intrinsically unfolded or natively unfolded proteins have been discovered and predicted to form a large fraction of eukaryotic proteins with various functions (Baldwin 2002, Rose 2002, Fink 2005). Together with its functionality, understanding physico-chemical properties of the unfolded protein is essential due to that the unfolded state of a protein serves as the starting conformation of the folding pathway and that partially or completely unfolded conformations are also key intermediates in amyloid formation.

\subsubsection{Conformational restrictions in unfolded proteins}

Consecutive amino acids are linked by peptide bonds forming a dihedral angle of $\sim 180^{\circ}$ between $\mathrm{H}-\mathrm{N}-\mathrm{C}_{\alpha}-\mathrm{O}$ with the exception of the rare occurrence of cis-proline (Berg et al. 2002). The degrees of freedom of polypeptide chain stems from the rotation of single bonds in the backbone. The backbone of a peptide chain consists of repeating

units of the three atoms $\mathrm{N}, \mathrm{Ca}$ and $\mathrm{C}$. While the dihedral angle along $\mathrm{C}-\mathrm{N}$ bond 
( $\omega$ angle) is limited around $180^{\circ}$ due to partial double bond character, $N-C_{\alpha}$ bond $(\varphi$ angle) and $\mathrm{C}_{\alpha}-\mathrm{C}$ bond ( $\psi$ angle) are single bonds with free rotation. In reality, $\varphi$ and $\psi$ dihedral angles in protein backbone are not freely rotatable but are restricted within a specified range by the steric hindrance of methyl or methylene group at the $\beta$ position. The specified dihedral angle space named Ramachandran plot was developed based on the theoretical study on Van der Waals interactions in dipeptide (Ramachandran 1963) and has been served as an important quality criterion for protein structure.

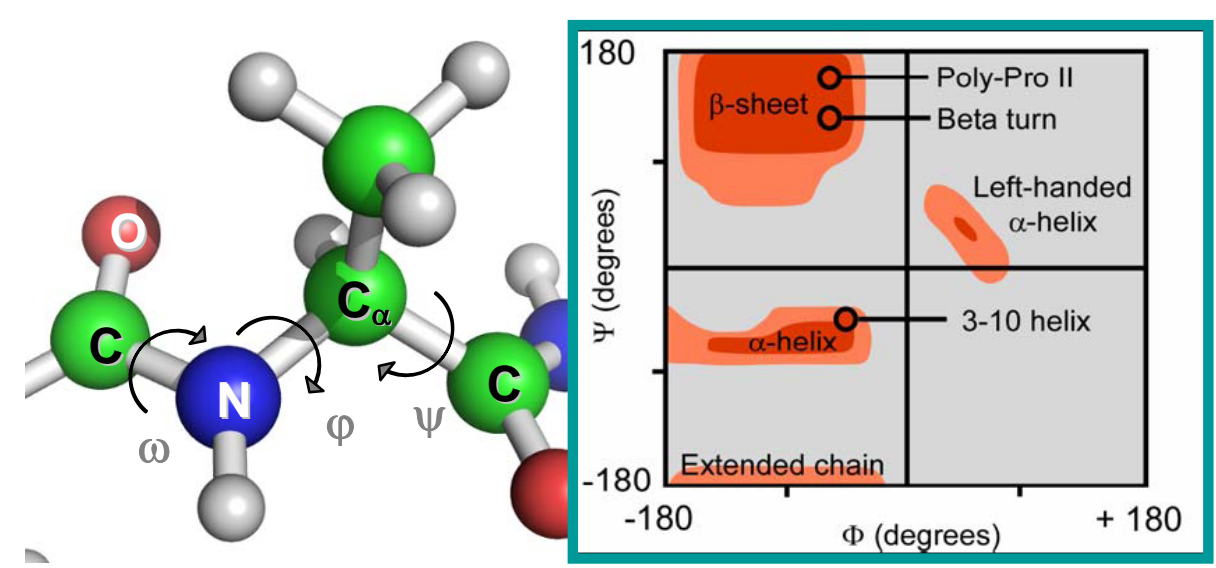

Fig. 1- 2. Dihedral angles in polypeptide chain and Ramachandran plot.

\subsubsection{In vitro methods for the characterization of unfolded protein}

The conformational heterogeneity of IUP hampered classical structure determination methods. In the last decades, however, various in vitro methods have been applied to characterize disordered proteins. Comprehensive reviews can be found elsewhere (Rose 2002, Eliezer 2007, Mittag and Forman-Kay 2007).

Table 1- 1. Biophysical methods for the unfolded proteins.

\begin{tabular}{l|l} 
Method & Information content \\
\hline \hline Analytical ultracentrifuge & Sedimentation coefficient $\rightarrow$ shape, multimeric state. \\
Dynamic light scattering & Diffusion coefficient $\rightarrow$ hydrodynamic radius.
\end{tabular}




\begin{tabular}{l|l} 
Small angle scattering & $\begin{array}{l}\text { Anisotropy of molecular shape } \\
\text { Neutron, X-ray } \rightarrow \text { Radius of gyration. } \\
\text { Anisotropy of molecular shape }\end{array}$ \\
Circular dichroism & Secondary structure content. \\
FTIR spectroscopy & Secondary structure content. \\
Raman spectroscopy & Secondary structure content. \\
EPR spectroscopy & Local order, distances through couplings. \\
Fluorescence spectroscopy & FRET $\rightarrow$ distance distribution between dyes. \\
NMR & Anisotropy $\rightarrow$ size changes. \\
Secondary chemical shift $\rightarrow$ secondary structure content. \\
J coupling $\rightarrow$ dihedral angle. \\
NOE $\rightarrow$ distances between two protons ( $~ 5 \AA)$. \\
PRE $\rightarrow$ distances from a spin label to a proton $(20 \sim 25 \AA)$. \\
Relaxation $\rightarrow$ local dynamics. \\
RDC $\rightarrow$ ensemble averaged orientation and dynamics. \\
DOSY $\rightarrow$ diffusion coefficient \& hydrodynamic redius.
\end{tabular}

\subsection{Protein misfolding and disease}

\subsubsection{Protein misfolding and disease}

A biological organism controls its cellular functions with proteins; expression and elimination of specific proteins (Radford and Dobson 1999). The proper regulation of folding and unfolding of specific proteins is essential for all biological system and, therefore, failure to fold correctly or to obtain proper stability will induce the malfunction of living systems and develop diseases in consequence (Dobson 2003). Some of these diseases result from incorrect folding of specific proteins and lose of its functionality, while the others are induced by toxic functions of misfolded proteins that are usually accompanied by aggregates form of specific proteins (Luheshi et al. 2008). An increasing number of disorders, including Alzheimer's disease, Parkinson's diseases, 
and the spongiform encephalopathies are directly associated with the deposition of such aggregates in tissues of various organs (Dobson 2003).

\subsubsection{Protein misfolding in Parkinson's disease}

Parkinson's disease (PD) is a progressive, neurodegenerative, and age-related movement disorder affecting more than $1 \%$ of the population over 65 years of age (Goedert 2001). It arises from the loss of dopaminergic neurons in the substantia nigra pars compacta in the brain and is accompanied by abnormal aggregates of protein inclusions in neuronal cells, which is known as Lewy bodies (LBs) and Lewy neuritis (Shults 2006).

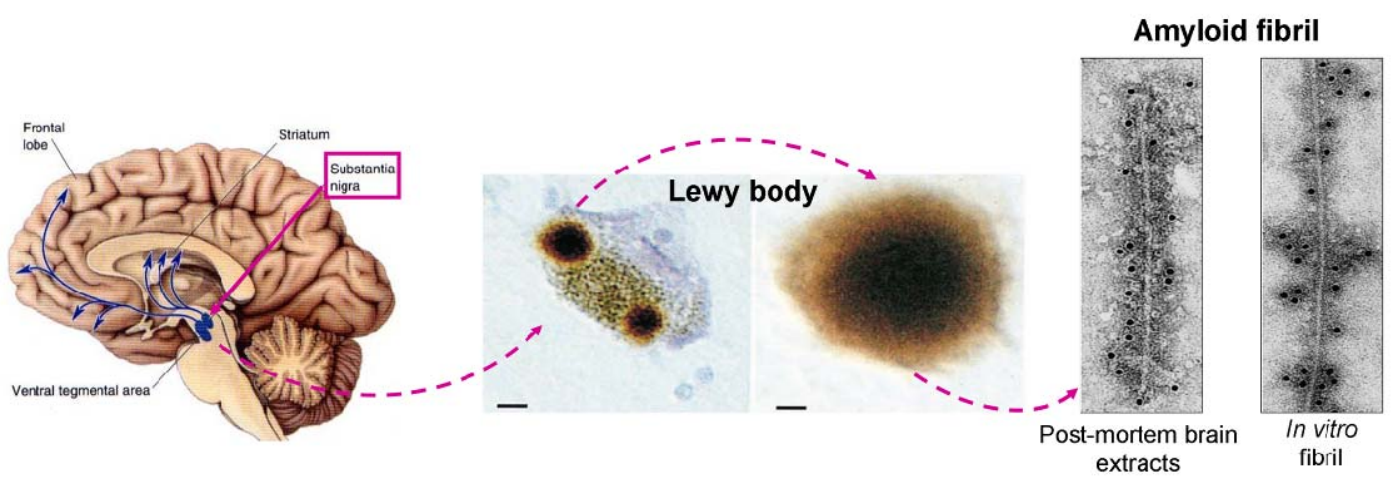

Fig. 1- 3. $\alpha \mathrm{S}$ aggregates in vivo and in vitro.

Dopamine pathway in the brain (left). In PD brain, Lewy bodies (LBs) are found in substantia nigra (middle).(Spillantini et al. 1997) The main component of LB is protein aggregates, mainly amyloid fibril form of $\alpha \mathrm{S}$, which could be produced in vitro (right).(Goedert 2001)

LBs are also found in a number of other neurodegenerative disorders including LB dementia (LBD); Alzheimer's disease (AD), including cases of familial AD with mutations in the presenilin 1, 2 and amyloid precursor protein genes; Down's syndrome; 
neurodegeneration with brain iron accumulation type 1 (also known as HallervordenSpatz disease); progressive autonomic failure; rapid eye movement sleep disorder; Parkinsonism-dementia complex of Guam; Gaucher's disease; and Pick's disease (Shults 2006). In electron microscope, LBs are densely packed proteins in the form of filaments and the major component of which is $\alpha$-synuclein $(\alpha \mathrm{S})$ (Spillantini et al. 1997). Other predominantly found proteins are heat shock proteins, neurofilaments, tau, synphilin-1, and tubulin (Shults 2006).

\subsubsection{Conformational change of $\alpha$-synuclein}

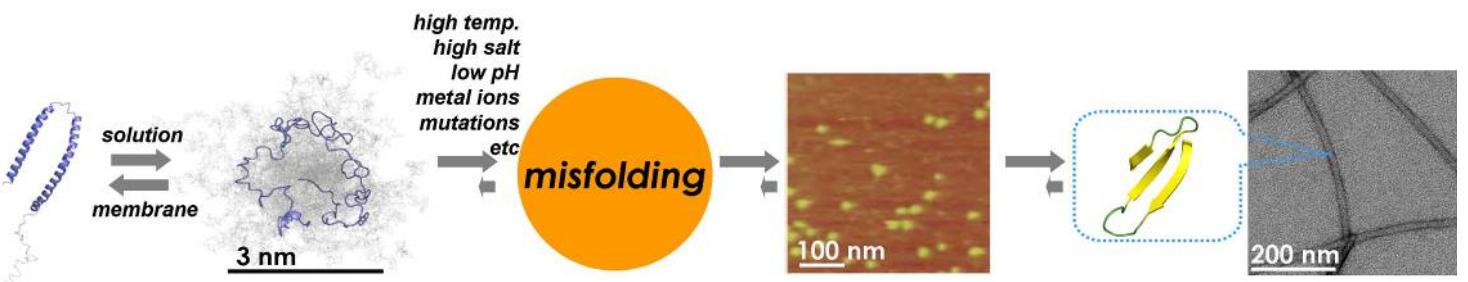

Fig. 1- 4. Schematic view of conformational change of $\alpha \mathrm{S}$.

At monomer state, $\alpha \mathrm{S}$ interacts with membrane and forms $\alpha$-helical structure. Under certain conditions, $\alpha \mathrm{S}$ aggregates and forms oligomers intermediate, and then is confined into a stable cross- $\beta$ structure in amyloid fibrils.

A schematic model of the conformation change of $\alpha \mathrm{S}$ in PD pathogenesis is shown in Fig. 1- 4. While $\alpha \mathrm{S}$ is found as an intrinsically unfolded protein in physiological condition, it adopts helical conformation upon binding with membrane (Ulmer et al. 2005). On the pathway to aggregation, it transforms into a spherical aggregates and turns into highly ordered $\beta$-strand structure in amyloid fibril (Uversky 2007). Together with its pathogenic role in $\mathrm{PD}$, this conformational change has attracted many 
researchers in biophysics and structural biology to understand protein misfolding and its toxicology, yet the exact mechanism at high resolution has not been clarified.

\subsection{NMR spectroscopy}

The basic phenomena of NMR that energy levels of atomic nuclear spins are split by a magnetic field and that transitions between these energy levels can be induced by irradiating with a frequency equal to the energy difference between the two levels, was discovered in 1946 (Bloch et al. 1946, Purcell et al. 1946). Since then, NMR spectroscopy has evolved enormously and become a powerful alternative tool for structure elucidation of biomolecule at atomic resolution. The advances in NMR spectroscopy were acknowledged by Nobel prizes in 1991 (Ernst 1992) for the 2D FT NMR and in 2002 for protein structure determination by NMR (Wuthrich 2003). At present, 7,500 of 52,500 biomolecular structures in PDB (www.pdb.org) (Berman et al. 2000) were determined by NMR spectroscopy. Distinguished from crystallography, NMR enables the researchers to study structures and dynamics of biomolecules in aqueous solution that is physiologically more relevant condition. A brief introduction to NMR parameters used for biomolecules is given below.

Table 1- 2. Physical constants used.

\begin{tabular}{|c|c|}
\hline Constants & Definition \\
\hline$\gamma\left(\gamma_{\mathrm{H}}\right)$ & gyromagnetic ratio (proton gyromagnetic ratio) \\
\hline$\hbar$ & Planck's constant divided by $2 \pi$ \\
\hline$\mu_{0}$ & Permeability of vacuum \\
\hline $\mathrm{B}_{0}$ & Static field strength \\
\hline$\omega\left(\omega_{\mathrm{H}}\right)$ & Larmor frequency (Proton Larmor frequency) \\
\hline $\mathrm{k}_{\mathrm{B}}$ & Boltzman constant. \\
\hline
\end{tabular}




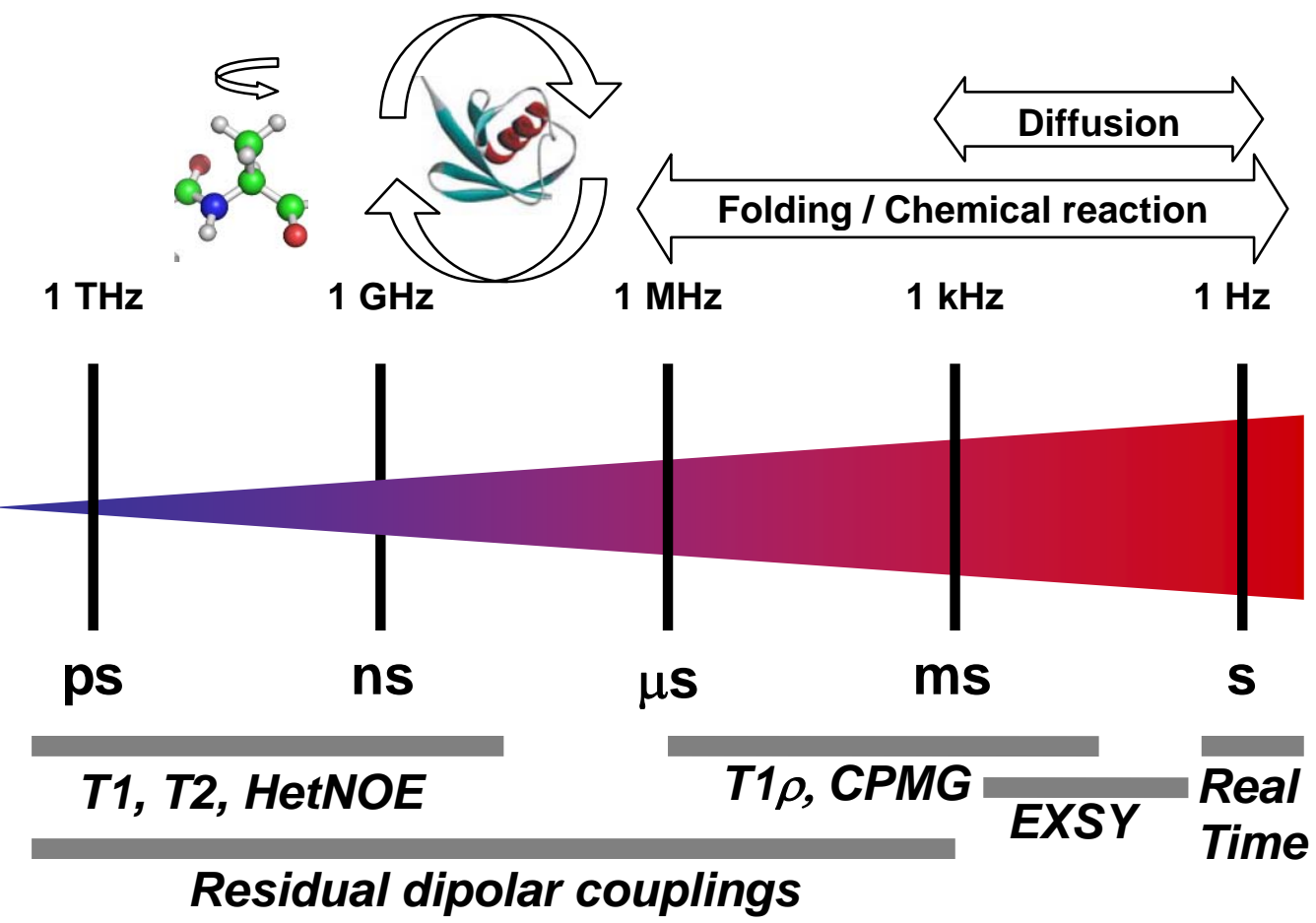

Fig. 1- 5. The time scale of protein dynamics and corresponding NMR parameters.

(adapted and modified from Lakomek et al. (Lakomek et al. 2006) and Palmer (Palmer 2004) )

\subsubsection{Chemical shift}

Chemical shift is a variation of Larmor frequency of atomic nucleus in a magnetic field (Levitt 2001, Cavanagh 2007). The total magnetic field experienced by a nucleus includes local magnetic fields induced by currents of electrons in the molecular orbitals as electrons have magnetic momentum. In protein NMR, the chemical shifts of ${ }^{13} \mathrm{C} \alpha$, ${ }^{13} \mathrm{C} \beta,{ }^{1} \mathrm{H} \alpha,{ }^{13} \mathrm{C}$, are sensitive to the protein secondary structure, and the difference $(\Delta \mathrm{CS})$ between the experimental chemical shifts $\left(\mathrm{CS}_{\text {exp. }}\right)$ and the random coil chemical shifts $\left(\mathrm{CS}_{\text {coil }}\right)$ are used for the secondary structure determination on a given sequence (Wishart and Sykes 1994). 


$$
\Delta C S=C S_{\text {exp. }}-C S_{\text {coil }}
$$

\subsubsection{Scalar coupling constant}

Scalar coupling is the indirect dipole-dipole coupling mediated by the electrons forming the chemical bonds between the nuclei (Levitt 2001, Cavanagh 2007). It modifies the energy levels of the systems, and changes NMR spectra accordingly. One and two-bond scalar coupling $\left({ }^{1} \mathrm{~J},{ }^{2} \mathrm{~J}\right)$ are heavily used in the heteronuclear solution state NMR to transfer the magnetization in multidimensional experiments (Sattler et al. 1999).

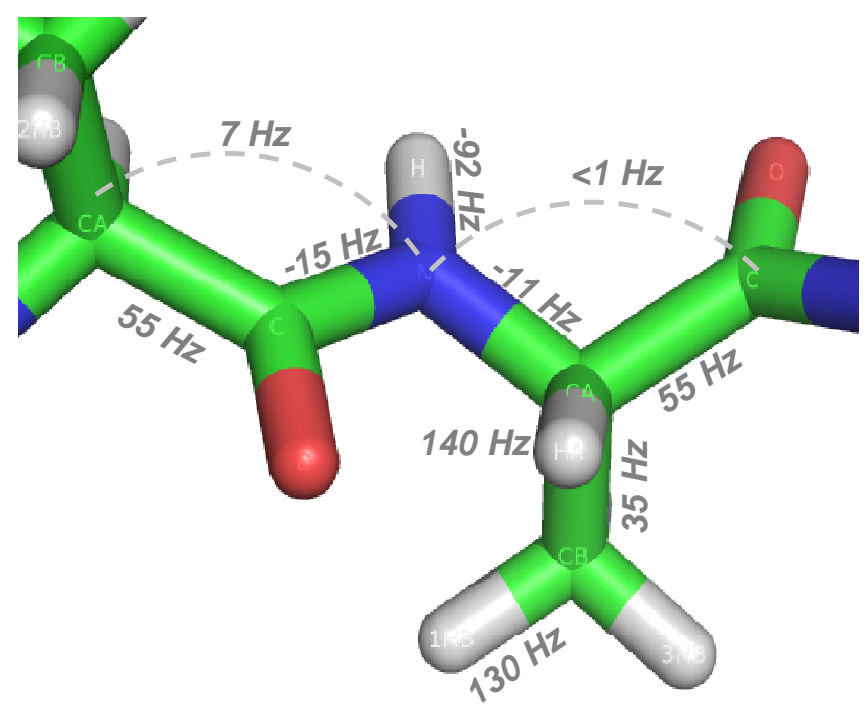

Fig. 1- 6. One-bond scalar coupling constants in peptide.

The usage of three-bond scalar coupling $\left({ }^{3} \mathrm{~J}\right)$ is to determine to the dihedral angle that can be estimated by Karplus equation (Eq. 1- 2) (Karplus 1963). The dihedral angle, then, can be interpreted into protein secondary structure using Ramachandran plot (Ramachandran 1963).

$$
J(\phi)=A \cos ^{2} \phi+B \cos \phi+C
$$

The protein secondary structure can be predicted directly from the ${ }^{3} \mathrm{~J}_{\mathrm{HNHA}}$ coupling 
constant by calculating the difference between the experimental coupling constant and the random coil value (Schwalbe et al. 1997).

\subsubsection{Nuclear Overhauser effect (NOE)}

NOE is a through-space spin-spin (inter-nuclear) interaction and described as crossrelaxation rate constants, $\sigma_{\mathrm{IS}}{ }^{\mathrm{NOE}}$. Using the isotropic rotor spectral density function, $\sigma_{\mathrm{IS}}{ }^{\mathrm{NOE}}$ for homonuclear spin system is given by (Eq. 1- 3).

$$
\sigma_{I S}^{N O E}=r_{I S}{ }^{-6} \times f\left(\tau_{c}\right)=\frac{\hbar^{2} \mu_{0}^{2} \gamma_{H}^{4} \tau_{c}}{160 \pi^{2} r_{I S}^{6}}\left(-1+\frac{6}{1+4 \omega_{H}^{2} \tau_{c}^{2}}\right)
$$

where $\quad \tau_{c}:$ the correlation time for the nuclear-nuclear interaction.

$\mathrm{r}_{\mathrm{IS}}: \quad$ the distance between spin I and spin S.

NOE is detectable when the two spins are close $(\sim 5 \AA)$ in space, and this makes NOE exceptionally useful for spatial proximity. Proton-proton NOEs are the most widely used parameter for the structure determination by NMR (Wüthrich 1986).

\subsubsection{Paramagnetic relaxation enhancement (PRE)}

Nitroxyl spin label, containing a paramagnetic unpaired electron, induces enhanced transverse relaxation of nearby nuclei, and this electron-proton interaction is detectable at a distance up to $20 \sim 25 \AA$, which gives a information of longer-range of interaction (Gillespie and Shortle 1997). The enhanced relaxation rate $\left(\mathrm{R}_{2, \text { para }}\right)$ is described as a function of electron-nuclear distance (r) as following (Kosen 1989): 


$$
R_{2, \text { para }}\left(\mathrm{s}^{-1}\right)=\frac{K}{r^{6}} \times\left(4 \tau_{c}+\frac{3 \tau_{c}}{1+\omega_{H}{ }^{2} \tau_{c}^{2}}\right)
$$

where $\quad \tau_{\mathrm{c}}:$ the correlation time for the electron-nuclear interaction.

$$
\mathrm{K}: \quad \text { composed of physical constant as } \frac{1}{15} S(S+1){\gamma_{H}}^{2} g^{2} \beta^{2}
$$

( $\mathrm{g}$, the electric $\mathrm{g}$ factor; $\beta$, Bohr magneton) and the value is $1.23 \times 10^{-32} \mathrm{~cm}^{6} \mathrm{~s}^{-2}$.

The paramagnetic effect is related to the intensity of amide cross peaks in $2 \mathrm{D}{ }^{1} \mathrm{H}^{-15} \mathrm{~N}$ HSQC spectra as following (Battiste and Wagner 2000):

$$
\frac{I_{\text {para }}}{I_{\text {dia }}}=\frac{R_{2, \text { dia }} \times e^{-R_{2, \text { para }} \cdot t}}{R_{2, \text { dia }}+R_{2, \text { para }}}
$$

where $\quad \mathrm{R}_{2 \text {,dia }}:$ the transverse relaxation rate of the amide proton w/o spin label.

$\mathrm{R}_{2, \text { para }}$ : the transverse relaxation rate of the amide proton with spin label. $I_{\text {para }} / I_{\text {dia }}$ : the intensity ratio for an amide cross peak between with spin label and without spin label.

$\mathrm{t}$ : the duration of INEPT delays $(\sim 10 \mathrm{~ms})$ in HSQC pulse sequence

The distance (r) can be extracted from above two equations and has been used for the generation of the $\alpha \mathrm{S}$ structural ensemble (Bertoncini et al. 2005b).

\subsubsection{Relaxations rates}

Relaxation is the process by which non-equilibrium magnetization returns to the equilibrium state (or transforms to another non-equilibrium state) (Jarymowycz and Stone 2006). Depending on the time scale of the dynamics, several methods have been 
developed and utilized as shown in Fig. 1- 5.

The overall methods for the protein dynamics study with the NMR relaxation parameters with backbone ${ }^{1} \mathrm{H}-{ }^{15} \mathrm{~N}$ bond vector have been reviewed in many literatures (Palmer 2004, Palmer et al. 2005, Jarymowycz and Stone 2006, Mittermaier and Kay 2006). All of these parameters are usually measured using two-dimensional HSQC-type experiments in which the intensities of cross peaks are modulated as a function of a delay time placed at a point in the sequence when the relevant relaxation process is active.

Here, a brief introduction of the NMR relaxation parameters to determine ps-ns dynamics is given below. All the relaxation rates are described based on the isotropic rotor spectral density function (Kempf and Loria 2003).

$$
J(\omega)=\frac{2 \tau_{e}}{5\left(1+\omega^{2} \tau_{e}^{2}\right)}
$$

where $\quad \tau_{\mathrm{e}}:$ the correlation time of the internal motion.

Reorientation of protein bond vectors, between spin I (i.e. ${ }^{1} \mathrm{H}$ ) and S (i.e. $\left.{ }^{15} \mathrm{~N}\right)$, on the ps-ns timescale contributes to the ${ }^{15} \mathrm{~N}$ longitudinal relaxation rate $\left(\mathrm{R}_{1}\right)$, the in-phase ${ }^{15} \mathrm{~N}$ transverse relaxation rate $\left(\mathrm{R}_{2}\right)$, and The ${ }^{1} \mathrm{H}^{-}{ }^{15} \mathrm{~N}$ heteronuclear NOE $\left(\sigma_{\mathrm{HN}}\right)$ as below.

$$
\begin{aligned}
R_{1}= & \left(D D^{2} / 4\right)\left[J\left(\omega_{I}-\omega_{S}\right)+3 J\left(\omega_{S}\right)+6 J\left(\omega_{I}+\omega_{S}\right)\right] \\
& +C S A^{2} J\left(\omega_{S}\right) \\
R_{2}= & \left(D D^{2} / 8\right)\left[4 J(0)+J\left(\omega_{I}-\omega_{S}\right)+3 J\left(\omega_{S}\right)+6 J\left(\omega_{I}\right)\right. \\
& \left.+6 J\left(\omega_{I}+\omega_{S}\right)\right]+C S A^{2}\left[4 J(0)+3 J\left(\omega_{S}\right)\right]+R_{e x}
\end{aligned}
$$




$$
\sigma_{H N}=\left(D D^{2} / 4\right)\left[6 J\left(\omega_{H}+\omega_{S}\right)-J\left(\omega_{H}-\omega_{S}\right)\right]
$$

where, DD: the magnitude of dipolar interaction. $\left(\mu_{0} h \gamma_{I} \gamma_{S} / 8 \pi^{2}\right) r_{I S}^{-3}$

CSA: the magnitude of CSA interaction. $\Delta \sigma_{S} / \sqrt{3}$

( $\Delta \sigma_{\mathrm{s}}$ is CSA of the S spin)

In the absence of chemical exchange (Rex), the spin relaxation concerns only motions of the interacting spins in Larmor frequencies, and sums or difference, which reflect psns time scale dynamics.

The ${ }^{15} \mathrm{~N}$ longitudinal relaxation rate in rotating frame $\left(\mathrm{R}_{1 \rho}\right)$ is described in terms of $\mathrm{R}_{1}$ and $\mathrm{R}_{2}$ as following.

$$
R_{1 \rho}=R_{1} \cos ^{2} \theta+R_{2} \sin ^{2} \theta
$$

where, $\quad \theta:$ the angle of the effective field with the rotating-frame z-axis. $\tan \theta=\omega_{1} / \Delta \Omega$ ( $\omega_{1}$, the amplitude the rf field; $\Delta \Omega$, the offset of the chemical shift from the rotating frame)

When $\theta$ is below $68^{\circ}$, the accuracy of $\tan \left(\Delta \Omega / \omega_{1}\right)=\left(\Delta \Omega / \omega_{1}\right)$ becomes lower than $95 \%$ and $R_{1 \rho}$ should be calibrated with $R_{1}$ to extract $R_{2}$.

\subsubsection{Residual dipolar coupling (RDC)}

In the last decade, one of the major developments in solution state NMR is the use of the anisotropic interactions like RDC, chemical shift anisotropy (CSA) and pseudo 
contact shifts (Tolman et al. 1995, Tjandra and Bax 1997, Prestegard 1998). Weak alignment of biomolecule in solution are induced by the paramagnetic property of the molecule (Tolman et al. 1995), or more generally by the use of a liquid crystal media cosolute (Tjandra and Bax 1997), and prevents complete averaging of the anisotropic interactions, while retaining the solution properties necessary for the high resolution NMR. Out of such anisotropic interactions, RDC is comparatively easy to measure and analyze.

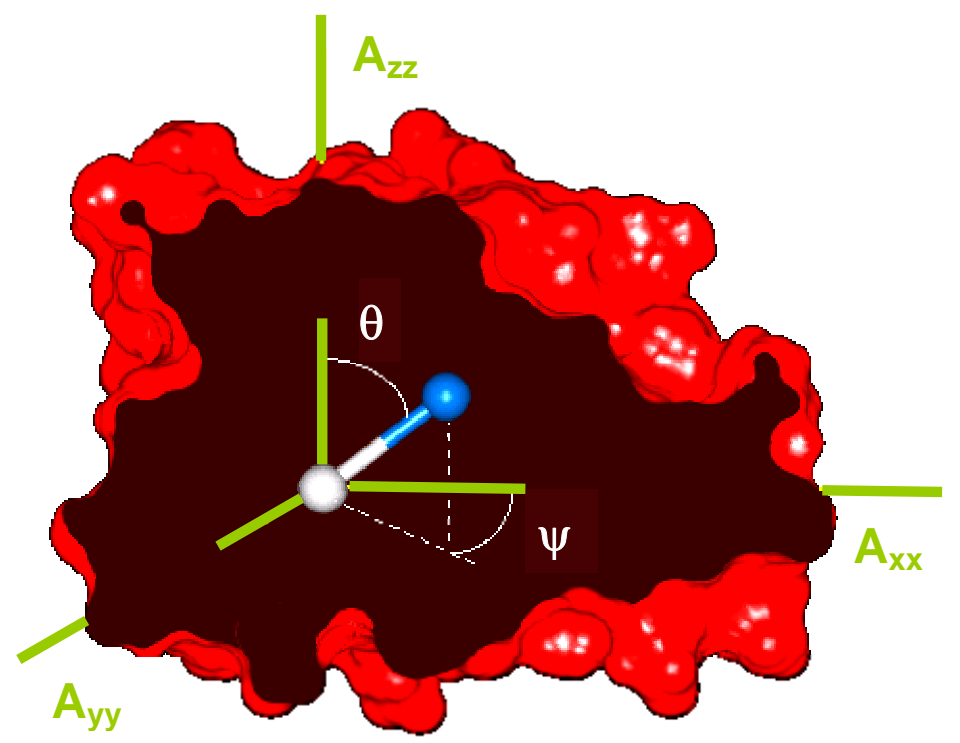

Fig. 1- 7. Schematic representation of the orientational dependence of RDC on the alignment tensor. The orientation of inter-spin vector $(\theta, \psi)$ with in the eigenframe of the alignment tensor, with eigenvalues Axx, Ayy and Azz.(adapted and modified from Blackledge (Blackledge 2005))

With the assumption that the motion is strictly local and exerts negligible influence on the molecular alignment tensor, and that the inter-nuclear distance averaging is again incorporated into an effective distance $\left(r_{i j, \mathrm{eff}}\right)$, RDC $\left(D_{i j}\right)$, determined in unit of $\mathrm{Hz}$, provides the mean orientation with respect to the alignment tensor, and the local 
dynamic averaging with respect to the mean orientation when (Eq. 1- 11) is transformed in terms of averaged spherical harmony (Blackledge 2005).

$$
\left\langle D_{i j}\right\rangle=-\frac{\gamma_{i} \gamma_{j} \mu_{0} h}{16 \pi^{3} r_{i j, e f f}^{3}}\left[A_{a}\left\langle 3 \cos ^{2} \theta-1\right\rangle+\frac{3}{2} A_{r}\left\langle\sin ^{2} \theta \cos 2 \psi\right\rangle\right]
$$

where $\quad \gamma_{i}, \gamma_{j}:$ gyromagnetic ratio of spin $i, j$

$\mathrm{A}_{\mathrm{a}}$ : Azz /2, Axial component of the alignment tensor

$A_{r}: \quad(1 / 3)(A x x-A y y)$, the rhombic component of the alignment tensor.

$\theta, \psi: \quad$ the inter-spin vector with respect to Azz for $\theta, \operatorname{Axx}$ for $\psi$

The angular brackets indicate averaging over all sampled conformations. The averaging reduces $\left\langle\mathrm{D}_{\mathrm{ij}}>\right.$ to zero in isotropic condition. Interpreted in terms of dynamics, $\left\langle\mathrm{D}_{\mathrm{ij}}>\right.$ covers ns to sub-ms time range dynamics of inter-spin vector (Lakomek et al. 2006, Lakomek et al. 2008).

\subsubsection{Diffusion ordered spectroscopy (DOSY)}

The Stokes-Einstein equation shows that the hydrodynamic radius $\left(\mathrm{R}_{\mathrm{h}}\right)$ of a protein is inversely proportional to the diffusion coefficient (D) of the protein as shown below.

$$
D=k_{B} T / 6 \pi \eta R_{h}
$$

where $\quad \mathrm{T}: \quad$ the temperature of the sample.

$\eta: \quad$ the viscosity of the solution

A tailored pulse-field gradient NMR (PFG-NMR) can detect the translational diffusion coefficient (D) of a molecule. Due to diffusion, molecules will change their spatial localization between the first and the second gradient pulses, and thus will 
experience an inhomogeneous total field, which causes an attenuation of the measured signal. For unrestricted diffusion of a molecule in an isotropic liquid, the PFG NMR signal amplitude (A) normalized to the signal obtained in the absence of gradient pulses, is related to the diffusion coefficient (D) by (Eq. 1- 13) (Stejskal and Tanner 1965).

$$
A=e^{-\left(\gamma^{2} \delta^{2} D(\Delta-\delta / 3) \times g^{2}\right.}
$$

where $\quad \mathrm{g}, \delta: \quad$ the magnitude and the duration of the field gradient pulse.

$\Delta: \quad$ the time between the gradient pulses

As shown in (Eq. 1- 13), the diffusion coefficient is affected by temperature and solvent viscosity, and thus the absolute value of the diffusion coefficient can be difficult to measure.

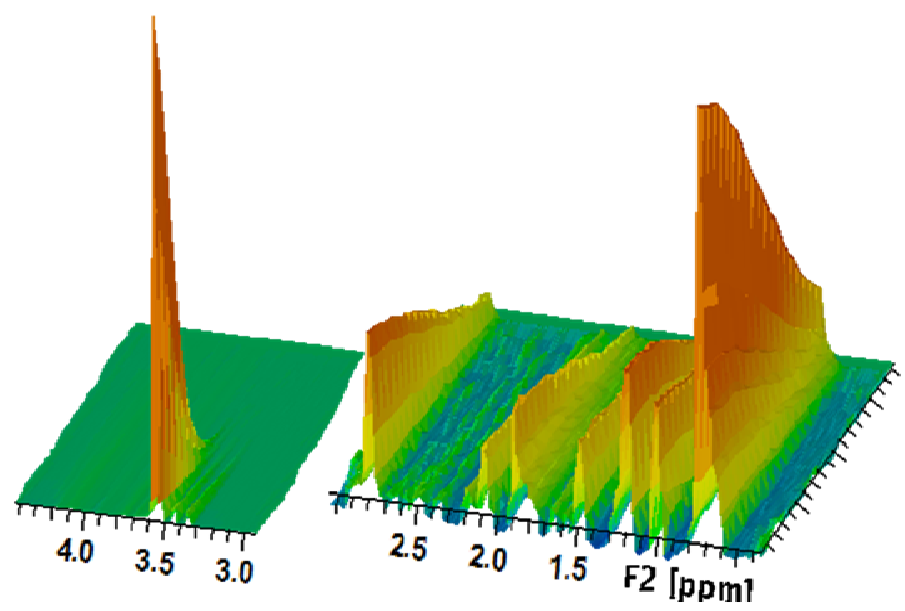

Fig. 1- 8. PG-SLED spectra of internal reference, dioxane, and $\alpha \mathrm{S}$.

The intensities decay as the gradient strength increase from $2 \%$ to $95 \%$ of the maximum value; the signal from the reference molecule, left panel; the signal from aliphatic region of target protein, right panel. Figures were generated from Topspin 2.1.

Jones et al. and Wilkins et al. suggested a different approach; using a reference molecule in the protein solution (Jones et al. 1997, Wilkins et al. 1999). The intensities 
decay as a function of gradient strength was fitted with a single Gaussian curve with decay rate. The observed decay rate, for protein $d_{\text {prot}}$, and for reference $d_{\text {ref, }}$ is proportional to the diffusion coefficient (D) of each molecule. Combined with the known hydrodynamic radius of the reference $\left(\mathrm{R}_{\mathrm{h}}{ }^{\text {ref }}\right)$, the hydrodynamic radius of the protein $\left(\mathrm{R}_{\mathrm{h}}{ }^{\text {prot}}\right)$ can be calculated.

$$
R_{h}^{\text {prot }}=\frac{d_{r e f}}{d_{p r o t}} R_{h}^{r e f}
$$




\subsection{Outline of the thesis}

The dramatic change of $\alpha \mathrm{S}$ morphology in PD has been intensively investigated in the last decade. Many types of mechanisms not only for the $\alpha \mathrm{S}$ conformational changes in PD, but for other neurodegenerative diseases have been proposed, and all of them share a common step called misfolding, oligomerization, and fibril formation.

In this thesis, I have investigated $\alpha \mathrm{S}$ as a model protein of the transition mechanism between a monomeric, unfolded protein to cross- $\beta$ structure in amyloid fibril.

In Chapter 3, a theoretical study about the basic determinant for the diverse dynamics detected by RDCs is given. The Intrinsically unfolded proteins (IUPs) like $\alpha \mathrm{S}$, are flexible and dynamic along whole amino acid sequence. The RDCs of IUPs, however, have shown deviations from the RDC profile of random-coil, implying that there are different local dynamics along the amino acid sequence. The determinant for the deviation is investigated.

In chapter 4, NMR and other biophysical studies on an aggregation-prone conformation of $\alpha \mathrm{S}$ are described. The aggregation of $\alpha \mathrm{S}$ is accelerated by many additives and environmental factors including low $\mathrm{pH}$. At $\mathrm{pH} 3, \alpha \mathrm{S}$ is know to adopt partially folded conformation that may serve as a key step for the amyloid fibril formation. NMR spectroscopy is applied to characterize the conformation.

In chapter 5, HR-MAS and solution NMR study on the core region of wt and A30P mutant $\alpha \mathrm{S}$ amyloid fibril is shown. The core of amyloid fibrils is known to consist of cross- $\beta$ structure, i.e. highly ordered hydrogen-bonding network. Hydrogen/deuterium exchange with NMR, however, could find that there are also flexible regions in the core region of both wt and A30P mutant, and HR-MAS NMR confirmed such flexible regions. The distribution of $\beta$-strands and flexible loops in the fibril core region is 
similar in both $w t$ and A30P mutant.

In chapter 6 , heterogenic and diverse conformation of the oligomer, derived from amyloid fibril through incubation in supercooled solution, is investigated with various biophysical and physiological methods. 


\section{Materials and methods}

\subsection{Materials}

\subsubsection{Equipments and consumables}

All the equipments and consumables used in this work are listed in Table 2- 1.

Table 2- 1. Equipments and consumables

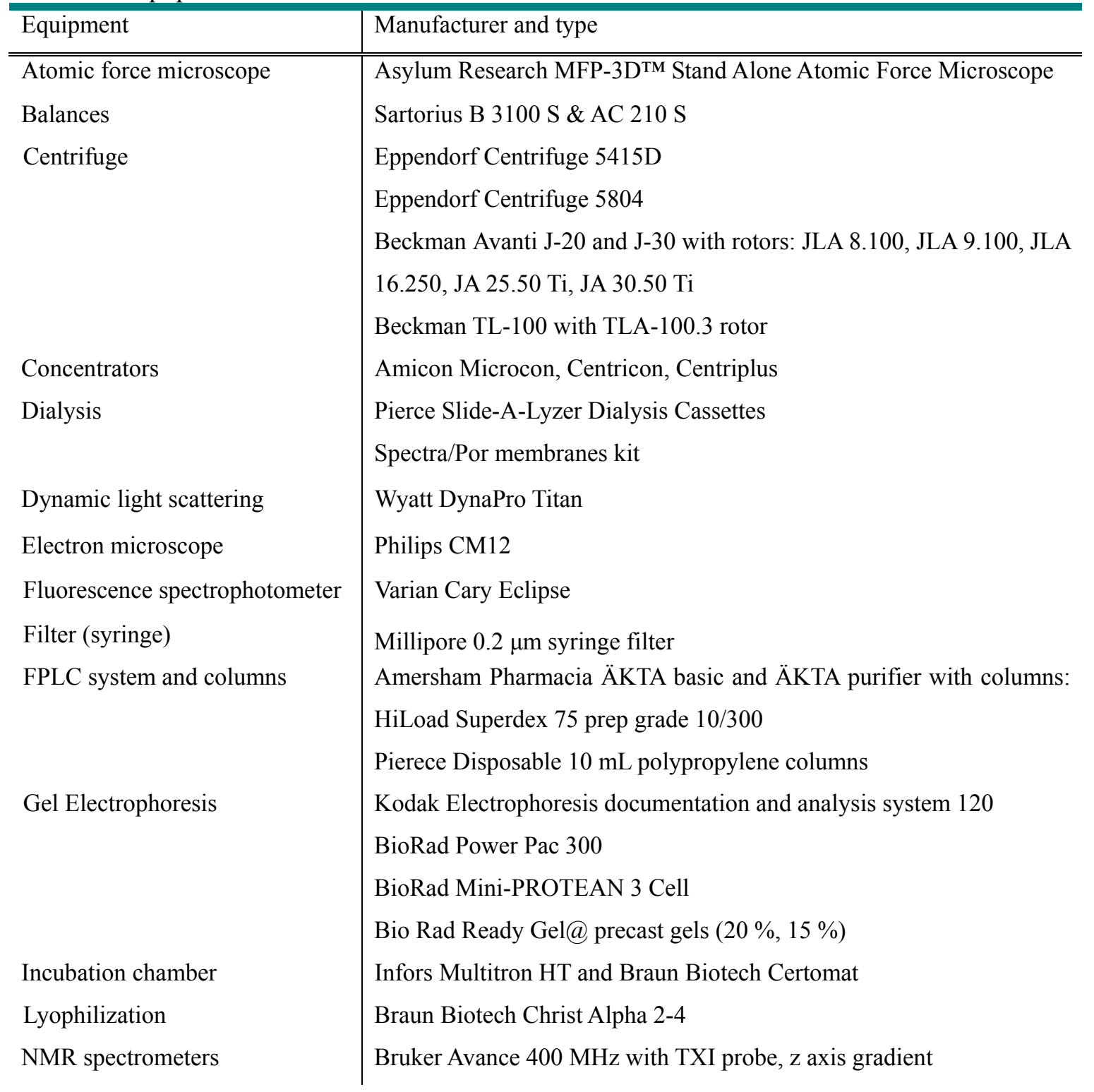


Peptide synthesizer

Sonicator

$\mathrm{UV}-\mathrm{Vis}$ spectrophotometer

UV-Vis CD spectrophotometer
Bruker DRX $600 \mathrm{MHz}$ with QXI probe, z axis gradient

Bruker Avance $600 \mathrm{MHz}$ with cryoprobe, z-axis gradient

Bruker Avance $700 \mathrm{MHz}$ with TXI probe, triple axis gradient

Bruker DRX $800 \mathrm{MHz}$ with TXI probe, triple axis gradient

Bruker Avance $900 \mathrm{MHz}$ with cryoprobe, z-axis gradient

Applied Biosystems 433A Peptide Synthesizer

Bandelin SONOPLUS HD 2200

Hewlett-Packard 8453

Jasco J-720

\subsubsection{Reagents}

All the reagents used in this work are listed in Table 2- 2.

Table 2- 2. Reagents

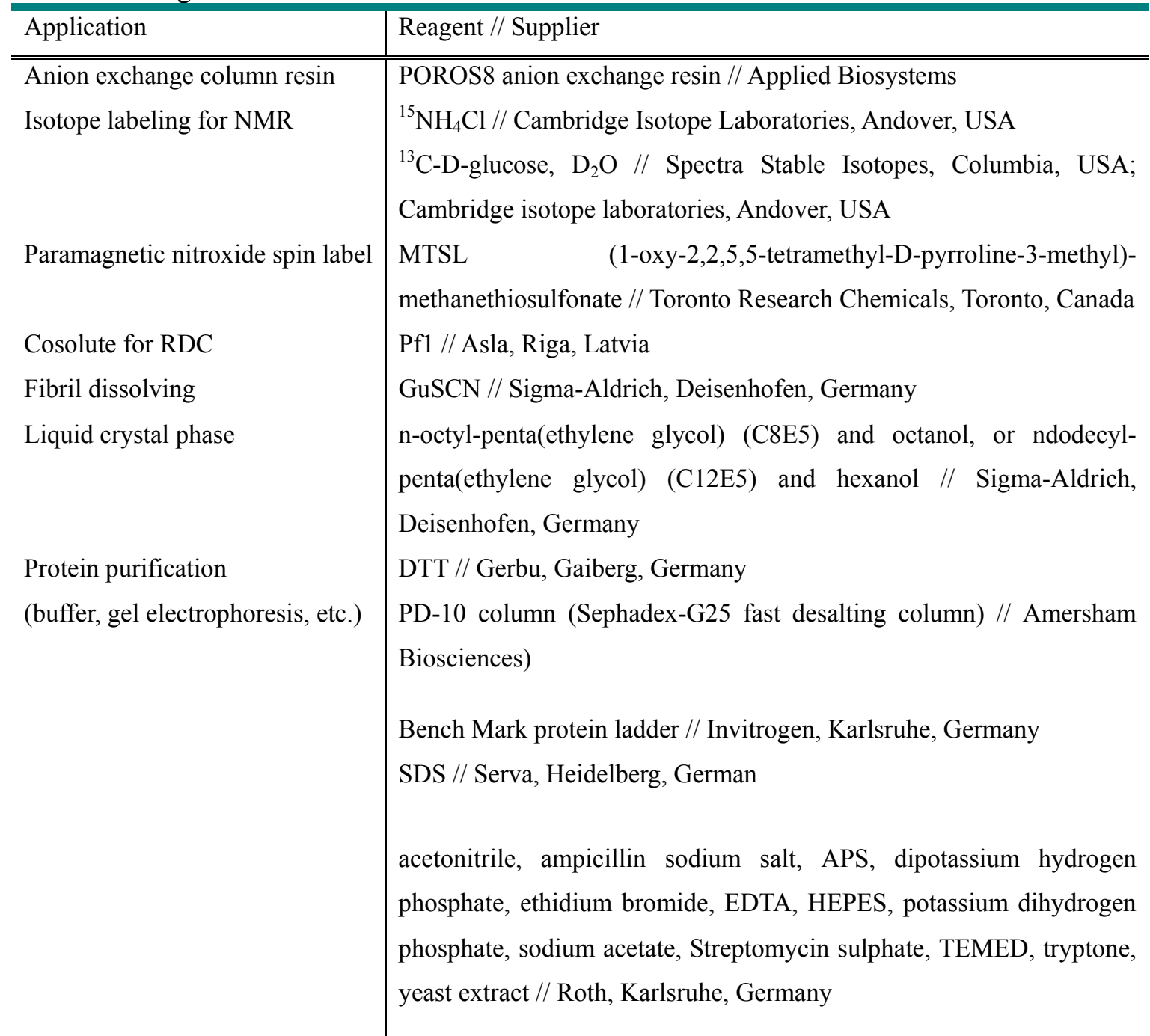


Complete $^{\mathrm{TM}}$ protease inhibitors, Complete ${ }^{\mathrm{TM}}$ protease inhibitors EDTA-free, elastase, protease K, trypsin // Roche Diagnostics, Mannheim, Germany acetic acid, $\alpha-\mathrm{D}(+)$-glucose monohydrate, ammonium acetate, ammonium chloride, ammonium hydrocarbonate, boric acid, disodium hydrogen phosphate, ethanol, formic acid, glycerol, glycine, hydrochloric acid, manganese chloride dihydrate, magnesium sulfate heptahydrate, sodium chloride, sodium dihydrogen phosphate, sodium hydroxide, TFA, thiaminechloride hydrochloride, Tris, urea // Merck, Darmstadt, Germany

Purified Mouse Anti- $\alpha$-Synuclein (BD) // BD Biosciences, Heidelberg, Germany

Rabbit anti-amyloid oligomer polyclonal antibody (A11) // Invitrogen, Biosource, Europe, S.A

\subsubsection{E. colicell culture media}

All the solution contents used for cultivation of E. coli cells are summarized in Table 2- 3. The culture media utilized in the expression of $\alpha \mathrm{S}$ were sterilised by autoclave. Prior to usage 100 $\mu \mathrm{g} / \mathrm{mL}$ ampicillin was added to each medium.

Table 2- 3. E.coli cell culture media contents

\begin{tabular}{l|lr} 
Name & Chemical & Amount \\
\hline \hline LB medium & Tryptone & $10 \mathrm{~g}$ \\
Meast extract & $5 \mathrm{~g}$ \\
$\mathrm{NaCl}$ & $10 \mathrm{~g}$ \\
$\mathrm{H}_{2} \mathrm{O}$ & up to $1 \mathrm{~L}$ \\
$\mathrm{Na}_{2} \mathrm{HPO}_{4}$ & $6.8 \mathrm{~g}$ \\
$\mathrm{KH}_{2} \mathrm{PO}_{4}$ & $3 \mathrm{~g}$ \\
$\mathrm{NaCl}^{2}$ & $0.5 \mathrm{~g}$ \\
& $\mathrm{NH}_{4} \mathrm{Cl}_{\text {or }}{ }^{15} \mathrm{NH}_{4} \mathrm{Cl}$ & $1 \mathrm{~g}$ \\
& $\mathrm{D}-\mathrm{glucose}^{13} \mathrm{C}_{6}$-D-glucose & $4 \mathrm{~g}$ \\
& $1 \mathrm{M} \mathrm{MgSO}_{4}$ & $2 \mathrm{~mL}$ \\
& $2 \mathrm{M} \mathrm{CaCl}_{2}$ & $50 \mu \mathrm{L}$ \\
& thiaminechloride hydrochloride & $0.03 \mathrm{~g}$ \\
& trace elements & $10 \mathrm{~mL}$ \\
& $\mathrm{H}_{2} \mathrm{O}$ & up to $1 \mathrm{~L}$
\end{tabular}




\begin{tabular}{l|lr} 
Trace elements & $\mathrm{FeSO}_{4} \cdot 7 \mathrm{H}_{2} \mathrm{O}$ & $0.6 \mathrm{~g}$ \\
$\mathrm{MnCl}_{2} \cdot 2 \mathrm{H}_{2} \mathrm{O}$ & $0.094 \mathrm{~g}$ \\
$\mathrm{CoCl}_{2} \cdot 6 \mathrm{H}_{2} \mathrm{O}$ & $0.08 \mathrm{~g}$ \\
$\mathrm{ZnSO} \cdot 7 \mathrm{H}_{2} \mathrm{O}$ & $0.07 \mathrm{~g}$ \\
$\mathrm{CuCl}_{2} \cdot 2 \mathrm{H}_{2} \mathrm{O}$ & $0.03 \mathrm{~g}$ \\
$\mathrm{H}_{3} \mathrm{BO}_{3}$ & $0.002 \mathrm{~g}$ \\
& $\left(\mathrm{NH}_{4}\right) 6 \mathrm{Mo}_{7} \mathrm{O}_{24} \cdot 4 \mathrm{H}_{2} \mathrm{O}$ & $0.025 \mathrm{~g}$ \\
Ampicillin stock sol. & $\mathrm{H}_{2} \mathrm{O}$ stir 10 min. & up to $100 \mathrm{~mL}$ \\
IPTG stock solution & EDTA stir over night & $0.5 \mathrm{~g}$ \\
\hline
\end{tabular}

\subsubsection{Software}

All the software used for NMR experiment, structure visualization, data analysis, mathematical calculation is summarized in Table 2- 4 .

Table 2- 4. Software

\begin{tabular}{l|l}
\hline Program & Reference \\
\hline \hline X-WINNMR 3.5 & Bruker Biospin, Karlsruhe, Germany \\
Topspin V1.0 2.1 & Bruker Biospin, Karlsruhe, Germany \\
NMRPipe/NMRDraw & spin.niddk.nih.gov/NMRPipe (Delaglio et al. 1995) \\
Sparky 3 & www.cgl.ucsf.edu/home/sparky (Goddard and Kneller) \\
PALES & www.mpibpc.mpg.de/groups/griesinger/zweckstetter/_links/software_pales.htm \\
& (Zweckstetter and Bax 2000,ZZweckstetter 2008) \\
Pymol & DeLano Scientific LLC, Palo Alto, CA, USA \\
MATHEMATICA & Wolfram Research, Champaign, IL, USA \\
ORIGIN 7.5 & OriginLab Corporation, Northampton, MA, USA \\
\hline
\end{tabular}

\subsection{Methods}

\subsubsection{Protein biochemistry}

\subsubsection{Protein expression and cultivation of $E$. coli}

All the solutions used for the protein expression were listed in Table 2- 3. The E. coli cell 
stocks containing wt $\alpha \mathrm{S}, \mathrm{C}$-terminal truncated wt $\alpha \mathrm{S}(\alpha \mathrm{S}(1-108))$ and Cys-mutant proteins were from Dr. Carlos Bertoncini at the Department of Molecular Biology, MPI-BPC.

E. coli (BL 21) cells containing T7 vector plasmid with $\alpha \mathrm{S}$ gene were grown overnight in $10 \mathrm{~mL}$ of $\mathrm{LB}$ medium at $37^{\circ} \mathrm{C}$ with shaking. The pre-culture was diluted $(10 \mathrm{~mL}$ in $1 \mathrm{~L} \mathrm{LB}$ medium) and allowed to grow at $37^{\circ} \mathrm{C}$ until OD600 of 0.6-0.8. Expression of the desired gene was then induced with $1 \mathrm{mM}$ IPTG. After $3 \sim 4$ hours of incubation at $37{ }^{\circ} \mathrm{C}$ with shaking cells were harvested by centrifugation at 3,000 g for 30 minutes. The harvested cells were frozen with liquid nitrogen and stored at $-80{ }^{\circ} \mathrm{C}$ till the further step.

Preparations of ${ }^{15} \mathrm{~N}$ - and ${ }^{13} \mathrm{C} /{ }^{15} \mathrm{~N}$ labeled proteins were carried out exactly as that of the non-labeled proteins with the difference that LB medium was replaced by M9 minimal medium supplemented with ${ }^{15} \mathrm{NH}_{4} \mathrm{Cl}$ or ${ }^{15} \mathrm{NH}_{4} \mathrm{Cl}$ and ${ }^{13} \mathrm{C}$-glucose.

\subsubsection{Protein purification}

The bacterial pellet was resuspended in $20 \mathrm{~mL}$ of $10 \mathrm{mM}$ Tris- $\mathrm{HCl}, \mathrm{pH} 8.0,1 \mathrm{mM}$ EDTA and $1 \mathrm{mM}$ PMSF, and lysed by multiple freeze-thaw cycles and following sonication. The cell lysate was boiled for 20 minutes and centrifuged at 20,000 g (30 minutes at $\left.4{ }^{\circ} \mathrm{C}\right)$ in order to precipitate the heat-denatured proteins (synucleins remain soluble). DNA was precipitated by the addition of streptomycin sulphate to the supernatant from the previous step (final concentration $10 \mathrm{mg} / \mathrm{mL}$ ). The mixture was stirred at $4{ }^{\circ} \mathrm{C}$ for 15 minutes and centrifuged as above described. Synuclein protein was then precipitated by addition of ammonium sulphate (final concentration $361 \mathrm{mg} / \mathrm{mL}$ ) to the supernatant from previous centrifugation. The solution was stirred at $4{ }^{\circ} \mathrm{C}$ for 15 minutes and centrifuged once again as above. The protein pellet was resuspended in $20 \mathrm{~mL}$ of $25 \mathrm{mM}$ Tris- $\mathrm{HCl}, \mathrm{pH} 7.7$, and loaded onto a POROS8 anion exchange column in ÄKTA Basic system equilibrated with 
$25 \mathrm{mM}$ Tris-HCl, $\mathrm{pH}$ 7.7. Full length $\alpha \mathrm{S}$ protein was eluted at a salt gradient of $\sim 300 \mu \mathrm{M}$ $\mathrm{NaCl}$. wt $\alpha \mathrm{S}(1-108)$ was eluted in the flow trough since the absence of acidic C-terminus impaired the ability of synuclein to bind to the column. The fractions containing protein (determined by UV spectroscopy and SDS-PAGE) were pooled together and concentrated using Centriplus YM-10 filter device (10,000 Da MWCO) to a final concentration of $\sim 300$ $\mu \mathrm{M}$. The concentrated proteins were dialyzed into the desired buffer listed in Table 2- 5 . The purification of Cys-mutants was similar with wt protein except that $1 \mathrm{mM}$ DTT was added to all the solutions used in order to prevent Cys oxidation and consequent dimerization.

Table 2- 5. Protein $\alpha \mathrm{S}$ samples used in this work.

\begin{tabular}{l|l} 
Usage & Solution \\
\hline \hline NMR \& EM at pH 7.4 & 20 mM Tris, $100 \mathrm{mM} \mathrm{NaCl}, \mathrm{pH} 7.4$ in $10 \% \mathrm{D}_{2} \mathrm{O} / 90 \% \mathrm{H}_{2} \mathrm{O}$ \\
$\mathrm{NMR} \& \mathrm{EM}$ at pH 7.4 & $20 \mathrm{mM}$ HEPES, $100 \mathrm{mM} \mathrm{NaCl}, \mathrm{pH} 7.4$ in $10 \% \mathrm{D}_{2} \mathrm{O} / 90 \% \mathrm{H}_{2} \mathrm{O}$ \\
$\mathrm{NMR} \& \mathrm{CD}$ at pH 7.4 & $20 \mathrm{mM} \mathrm{NaPi}, 100 \mathrm{mM} \mathrm{NaCl}, \mathrm{pH} 7.4$ in $10 \% \mathrm{D}_{2} \mathrm{O} / 90 \% \mathrm{H}_{2} \mathrm{O}$ \\
$\mathrm{NMR}$ at pH 3 & $20 \mathrm{mM}$ Na-acetate, $100 \mathrm{mM} \mathrm{NaCl}, \mathrm{pH} 3$ in $10 \% \mathrm{D}_{2} \mathrm{O} / 90 \% \mathrm{H}_{2} \mathrm{O}$ \\
Diffusion experiment & wtih or w/o $100 \mathrm{mM} \mathrm{NaCl}$ in $99.9 \% \mathrm{D}_{2} \mathrm{O}$
\end{tabular}

\subsubsection{C-terminal peptide}

The C-terminal $\alpha \mathrm{S}$ peptide was synthesized by Kerstin Overkamp at the Department of NMR-based Structural Biology, MPI-BPC. Standard solid-phase peptide synthesis was employed to produce peptides comprising residues 105 to 136 of wt $\alpha \mathrm{S}$. The peptides were purified by reverse phase HPLC and the purity ( $>95 \%)$ was analyzed by mass spectrometry.

\subsubsection{Spin-labeling of protein}

Previously developed Cys-mutants were used, which are A18C, A90C, and A140C wt $\alpha$ S. The reaction of $\alpha \mathrm{S}$ Cys-mutants with the nitroxide spin label MTSL (1-oxy-2,2,5,5- 
tetramethyl-D-pyrroline-3-methyl)-methanethiosulfonate was carried in the same way described by Bertoncini et al.(Bertoncini et al. 2005b)

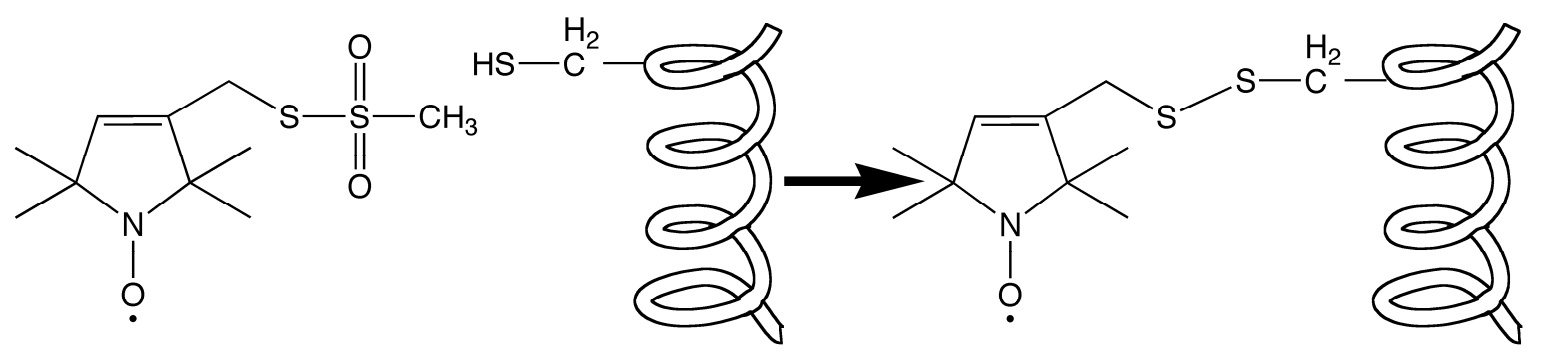

Fig. 2- 1. Reaction of MTSL with Cys residue in a protein.

Briefly, DTT was removed prior to labeling from the buffer using PD-10 column and the proteins were equilibrated in $20 \mathrm{mM}$ Tris-HCl, $\mathrm{pH}$ 7.4, $100 \mathrm{mM} \mathrm{NaCl}$. Free sulfhydryl groups were reacted with a 5-fold molar excess of the MTSL maleimide reagent solubilized in acetone, at $4{ }^{\circ} \mathrm{C}$ for $12 \mathrm{~h}$, in the dark. Unreacted spin labels were removed by dialysis against the desired buffer. Complete labeling with the nitroxide radical was verified by ESImass spectrometry. Proteins were aliquoted, fast frozen in liquid $\mathrm{N}_{2}$ and stored at $-80{ }^{\circ} \mathrm{C}$. The diamagnetic spectra were acquired 1 2 hour after addition of $1 \mathrm{mM}$ DTT (at pH 7.4) or ascorbic acid (at $\mathrm{pH} 3$ ) into MTSL- $\alpha \mathrm{S}$ sample.

\subsubsection{Protein aggregation assay}

Aggregation measurements of $\alpha \mathrm{S}$ were performed with $100 \mu \mathrm{M}$ protein samples in buffer (buffer A: 20 mM Tris-HCl, $100 \mathrm{mM} \mathrm{NaCl,} \mathrm{pH} \mathrm{7.4;} \mathrm{buffer} \mathrm{B:} 20$ mM HEPES, $100 \mathrm{mM}$ $\mathrm{NaCl}, \mathrm{pH} 7.4$ ) with addition of $0.01 \% \mathrm{NaN}_{3} .500 \mu \mathrm{L}$ of protein sample was incubated in glass vials at $37^{\circ} \mathrm{C}$ under constant stirring $(200 \mathrm{rpm})$ with magnetic microbars.

Determination of the amount of fibril formation at different time points was carried out 
by the Thioflavin-T (ThT) assay as described by Hoyer et al.(Hoyer et al. 2002). $5 \mu \mathrm{L}$ aliquots were withdrawn from the assay and diluted in $2 \mathrm{ml}$ of $50 \mu \mathrm{M}$ ThT in $50 \mathrm{mM}$ Glycine buffer, $\mathrm{pH}$ 8.0. ThT fluorescence was measured in a Varian Cary Eclipse spectroflourometer, with an excitation wavelength of $446 \mathrm{~nm}$ and emission wavelengths range of 460 to $600 \mathrm{~nm}$. Fluorescence at $480 \mathrm{~nm}$ was used for determination of the relative content of $\alpha \mathrm{S}$ fibrils in the sample.

\subsubsection{NMR spectroscopy}

$\sim 0.3 \mathrm{mM}{ }^{13} \mathrm{C} /{ }^{15} \mathrm{~N}$ labeled $\alpha \mathrm{S}$ sample was used for resonance assignment. For RDC and chemical shift perturbation, $\sim 0.1 \mathrm{mM}{ }^{15} \mathrm{~N} \alpha \mathrm{S}$ sample was used. Triple resonance 3D experiments were performed in either Bruker $900 \mathrm{MHz}$ NMR spectrometer equipped with TCI-Cryo probe or Bruker $600 \mathrm{MHz}$ NMR spectrometer equipped with TXI-Cryo probe. ${ }^{1} \mathrm{H}^{-}{ }^{15} \mathrm{~N}$ HSQC, IPAP-HSQC were measured in all NMR spectrometer listed in Table 2- 1. The acquired spectra were processed by XWIN-NMR, Topspin or NMRPipe including apodization with a squared sine function, zero-filling and/or forward linear prediction (Levitt 2001, Cavanagh 2007). The processed spectra were analyzed by Sparky or NMRPipe/NMRDraw listed in Table 2- 4.

\subsubsection{Resonance assignment}

Since $\alpha \mathrm{S}$ is an intrinsically unfolded protein, it was not feasible to apply the conventional NMR method for globular protein backbone resonance assignment using HNCACB, $\mathrm{CBCA}(\mathrm{CO}) \mathrm{NH}, \mathrm{HNCO}$ and $\mathrm{HN}(\mathrm{CA}) \mathrm{CO}$. Instead, backbone ${ }^{1} \mathrm{H}$ and ${ }^{15} \mathrm{~N}$ chemical shifts were assigned first with HNN (Panchal et al. 2001) experiment.. Regarding ${ }^{1} \mathrm{H}$ and ${ }^{15} \mathrm{~N}$ resonances assignments as a template, $\mathrm{C}_{\alpha}, \mathrm{C}_{\beta}$, and $\mathrm{C}^{\prime}$ resonances acquired from HNCACB, 
CBCA(CO)NH, IPAP-(HA)CANH, HNCO experiments were assigned.

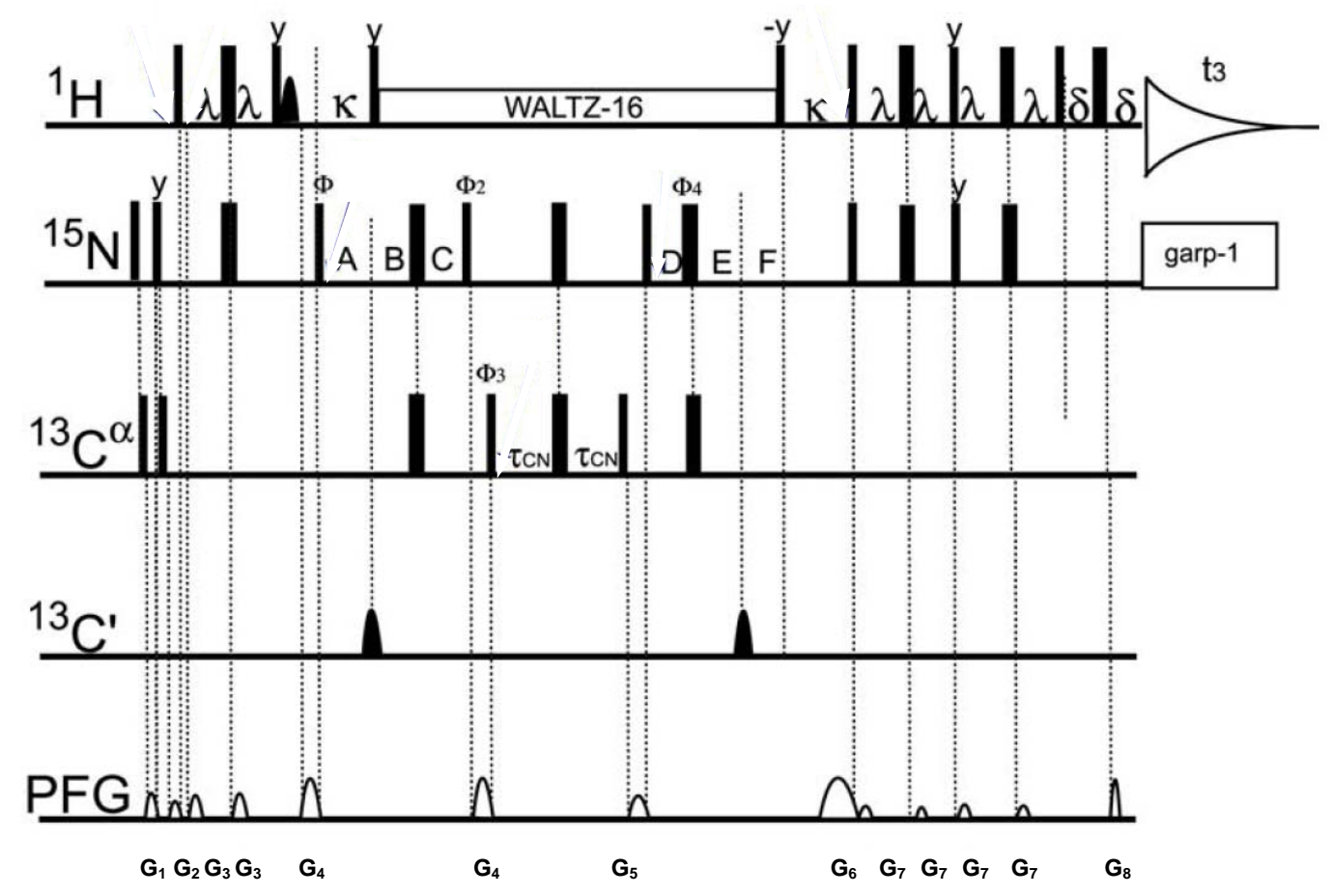

Fig. 2- 2. Pulse sequence of HNN.

Narrow and wide pulses correspond to flip angles of $90^{\circ}$ and $180^{\circ}$, respectively, with phase x unless indicated. The water flip-back during the first INEPT transfer is applied for duration of $1.0 \mathrm{~ms}$ with one-lobe sinc profile. Proton decoupling using Waltz-16 decoupling sequence is applied during most of the $t 1$ and $t 2$ evolution periods, and ${ }^{15} \mathrm{~N}$ decoupling using the Garp sequence is applied during acquisition. The delays are $\lambda=2.7 \mathrm{ms,}$ $\kappa=5.4 \mathrm{~ms}$ and $\delta=0.35 \mathrm{~ms} . \tau_{\mathrm{CN}}$ must be optimized and is around $12-16 \mathrm{~ms}$. Values of the individual periods in $\mathrm{t} 1$ are: $\mathrm{A}=\mathrm{t} 1 / 2, \mathrm{~B}=\mathrm{T}_{\mathrm{N}}$ and $\mathrm{C}=\mathrm{T}_{\mathrm{N}}-\mathrm{t} 1 / 2$. The values of the individual periods containing $\mathrm{t} 2$ are: $\mathrm{D}=$ $\mathrm{T}_{\mathrm{N}}-\mathrm{t} 2 / 2, \mathrm{E}=\mathrm{T}_{\mathrm{N}}$ and $\mathrm{F}=\mathrm{t} 2 / 2$. Phase cycling for the experiment is $\varphi=2(\mathrm{x}), 2(-\mathrm{x}) ; \varphi 2=\mathrm{x},-\mathrm{x}, \mathrm{x},-\mathrm{x} ; \varphi 3=\varphi 2$; $\varphi 4=x$; and receiver $=2(x), 2(-x)$. Frequency discrimination in $\mathrm{t} 1$ is achieved using States-TPPI phase cycling of $\varphi 1$ along with the receiver phase; frequency discrimination in $\mathrm{t} 2$ is achieved using the PEP sensitivityenhanced gradient method. The N- and P-type signals are collected separately by inverting the sign of the G6 gradient pulse. Signals recorded are manipulated post acquisition to generate pure absorption data. The gradient duration and levels are as follows: $\mathrm{G} 1=0.5 \mathrm{~ms}, 8 \mathrm{G} / \mathrm{cm}$; G2 $=0.5 \mathrm{~ms}, 5.6 \mathrm{G} / \mathrm{cm}$; G3 $=0.5 \mathrm{~ms}, 8$ $\mathrm{G} / \mathrm{cm}$; G4 = $1 \mathrm{~ms}, 11 \mathrm{G} / \mathrm{cm}$; G5 = $1.0 \mathrm{~ms}, 8 \mathrm{G} / \mathrm{cm}$; G6 $=2.5 \mathrm{~ms}, 26 \mathrm{G} / \mathrm{cm}$; G7 $=0.5 \mathrm{~ms}, 2 \mathrm{G} / \mathrm{cm}$; G8 $=0.25$ ms, $25.8 \mathrm{G} / \mathrm{cm}$. (adapted from Panchal et al, 2001(Panchal et al. 2001)) 


\subsubsection{Secondary structure determination}

Regions of secondary structure in protein can be identified by the difference between the experimental chemical shift $\left(\mathrm{CS}_{\mathrm{exp}}\right)$ and the random coil chemical shift $\left(\mathrm{CS}_{\text {coil }}\right)$. Sequence corrected random coil values determined by Schwarzinger et al.(Schwarzinger et al. 2001) or random coil value from statistical analysis by Wishart et al.(Wishart et al. 1995) were used. Secondary chemical shifts $(\Delta \mathrm{CS})$ for $\mathrm{C}_{\alpha}$ and $\mathrm{C}^{\prime}$ were calculated, respectively using (Eq. 1- 1).

\subsubsection{Chemical shift difference}

Chemical shifts depend on the local chemical environment of a nucleus. When ligand interaction or conformational changes exist, the chemical shifts of specific amino acids would be perturbed. These perturbations can be followed in every NMR spectra. For protein, ${ }^{1} \mathrm{H}-{ }^{15} \mathrm{~N}$ HSQC spectrum is used for widely due to high sensitivity. The difference in ${ }^{1} \mathrm{H}$ and ${ }^{15} \mathrm{~N}$ chemical shifts are commonly combined into an averaged value calculated as (Eq. 2- 1)

$$
\Delta \delta_{H N}=\sqrt{\frac{\left(\Delta \delta_{N} / 5\right)^{2}+\Delta \delta_{H}{ }^{2}}{2}}
$$

\subsubsection{Diffusion coefficient and hydrodynamic radius}

Diffusion-ordered spectroscopy (DOSY) with Pulse filed gradient NMR (PFG-NMR) was done with PG-SLED pulse program with inert internal standard, dioxane, as described in Wilkins et al.(Wilkins et al. 1999). 


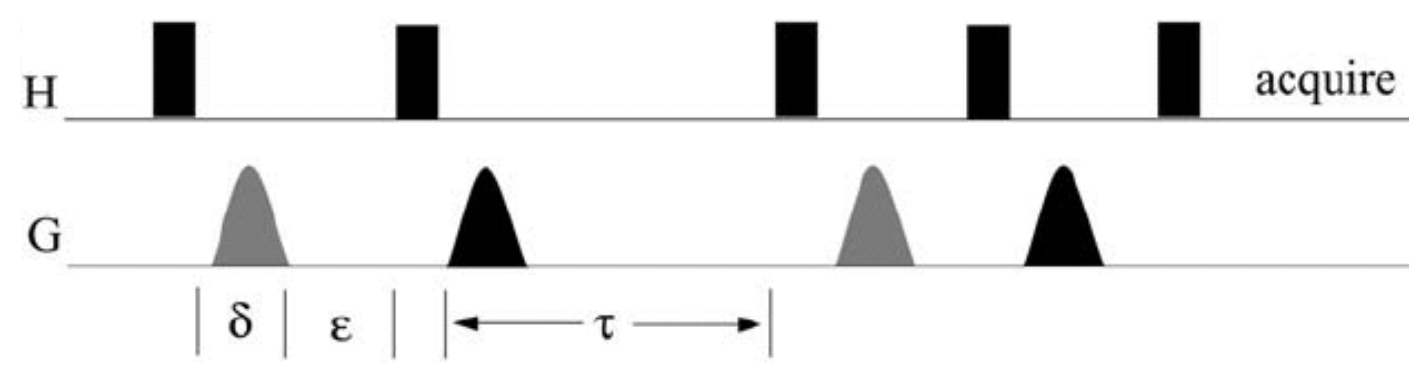

Fig. 2- 3. Pulse sequence of PG-SLED.

The rectangles correspond to ${ }^{1} \mathrm{H}$ flip angle of $90^{\circ}$. The phase cycles are: $\varphi=\mathrm{x}, \mathrm{x}, \mathrm{y}, \mathrm{y},-\mathrm{x},-\mathrm{x},-\mathrm{y},-\mathrm{y} ; \psi=\mathrm{x},-\mathrm{x}$, $\mathrm{y},-\mathrm{y},-\mathrm{x}, \mathrm{x},-\mathrm{y}, \mathrm{y}$. The delays are: $\delta=6.3 \mathrm{~ms}, \varepsilon=1.0 \mathrm{~ms}$ and $\tau=100 \mathrm{~ms}$. The diffusion labeling gradients, shown in grey, were varied (2 95\%), while the crush gradients, shown in black, were applied at full as strength (adapted and modified from Jones et al. (Jones et al. 1997)).

The signals of the protein corresponding to the aliphatic region and to the aromatic region were integrated, respectively. The intensity of the reference molecule is also integrated in the same way. The decay of the signals as a function of gradient strength was fitted to a Gaussian function. The obtained diffusion constant, proportional to diffusion coefficient, for both the protein $\left(d_{\text {prot }}\right)$ and the reference $\left(d_{r e f}\right)$, and the hydrodynamic radius of the reference $\left(\mathrm{R}_{\mathrm{h}}{ }^{\text {ref }}=2.12 \AA\right.$ for dioxane) were used in (Eq. 1- 14) to determine hydrodynamic radius of the protein $\left(\mathrm{R}_{\mathrm{h}}{ }^{\mathrm{prot}}\right)$.

\subsubsection{Longitudinal relaxation rate in rotating frame $(\mathrm{R} 1 \rho)$}

Residue specific ${ }^{15} \mathrm{~N}$ R1 $\rho$ was measured by Hai-Young Kim. A conventional R1 $\rho$ experiment was performed by collecting series of 2D spectra with different relaxation delays $(8,32,48,88$ and $176 \mathrm{~ms})$ with the on-resonance spin-lock pulse at $2.5 \mathrm{kHz}$ that makes a tilt angle bigger that $68^{\circ} \mathrm{C}$ for the ${ }^{15} \mathrm{~N}$ magnetization in the desired spectral width (Cavanagh 2007). The peak intensity decay of each residue as a function of the delay time was fitted with single exponential curve and the corresponding R1 $\rho$ was acquired. 


\subsubsection{Residual dipolar coupling (RDC)}

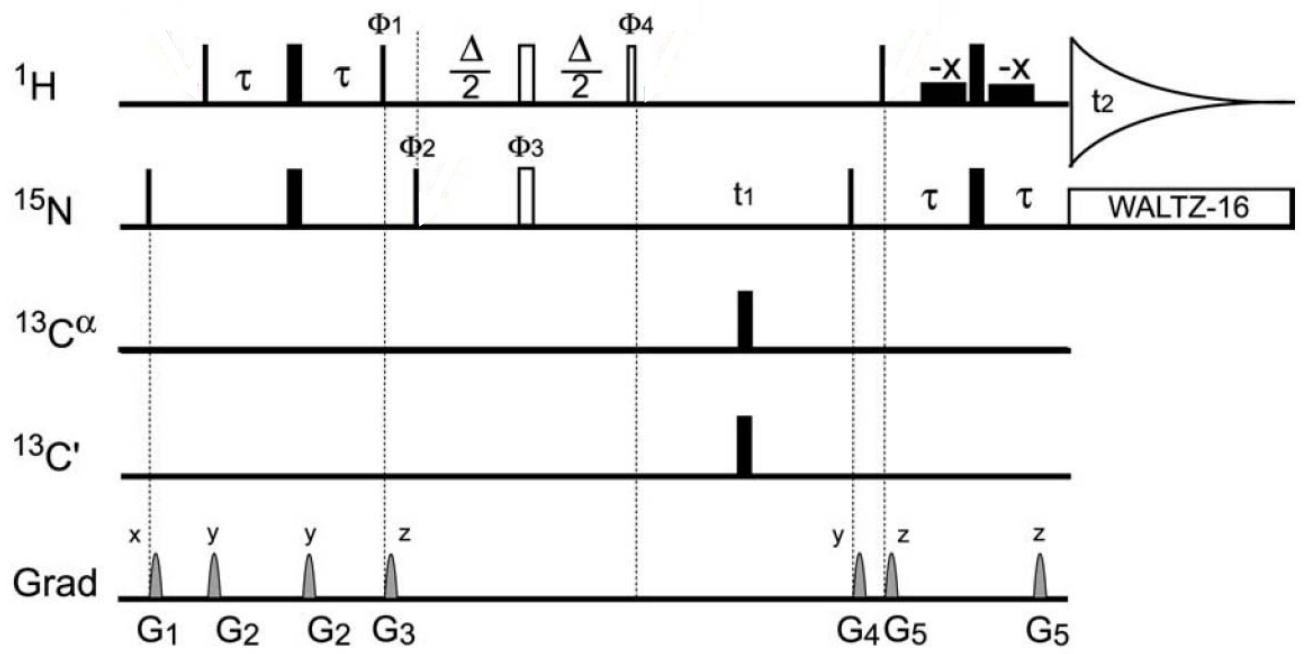

Fig. 2- 4. Pulse sequence of IPAP- ${ }^{1} \mathrm{H},{ }^{15} \mathrm{~N}-\mathrm{HSQC}$.

Narrow and wide pulses correspond to flip angles of $90^{\circ}$ and $180^{\circ}$, respectively, with phase $\mathrm{x}$ unless indicated. The IPAP-element is shown as open rectangles. WATERGATE-solvent suppression element(Piotto et al. 1992) is used for water suppression. ${ }^{13} \mathrm{C}^{\prime}$ and ${ }^{13} \mathrm{C}_{\alpha} 1800^{\circ}$ pulses decouple ${ }^{13} \mathrm{C}$ from ${ }^{15} \mathrm{~N}$ t1 evolution. Delays: $\tau=$ $2.7 \mathrm{~ms} ; \Delta=5.3 \mathrm{~ms}$. Phase cycles: $\varphi 1=-\mathrm{y}, \mathrm{y} ; \varphi 2=2(\mathrm{x}), 2(-\mathrm{x})$ for IP; $\varphi 2=2(-\mathrm{y}), 2(\mathrm{y})$ for AP; $\varphi 3=4(\mathrm{x}), 4(\mathrm{y})$, 4(-x), 4(-y), 4(-y); $\varphi 4=8(\mathrm{x}), 8(-\mathrm{x}) ;$ prec =x, 2(-x), x for IP; $\varphi$ rec =x, 2(-x), x, -x, 2(x), -x for AP. (adapted from Ottiger et al.(Ottiger et al. 1998))

Spectra of ${ }^{15} \mathrm{~N}$ and/or ${ }^{13} \mathrm{C}$ labeled $\alpha \mathrm{S}$ sample in isotropic condition were measured and then same spectra were acquired after alignment cosolute, Pf1 or C8E5/Octanol, was added.

Backbone ${ }^{1} \mathrm{D}_{\mathrm{NH}}$ was determined with IPAP- ${ }^{1} \mathrm{H},{ }^{15} \mathrm{~N}-\mathrm{HSQC}$ pulse program shown in Fig. 2- 4 (Ottiger et al. 1998).

${ }^{1} \mathrm{D}_{\mathrm{C} \alpha \mathrm{H} \alpha}$ were obtained by measuring IPAP-(HA)CANH (Zweckstetter and Bax 2001) spectra of $13 \mathrm{C} / 15 \mathrm{~N}-$ labeled $\alpha \mathrm{S}$ under isotropic and anisotropic conditions. 


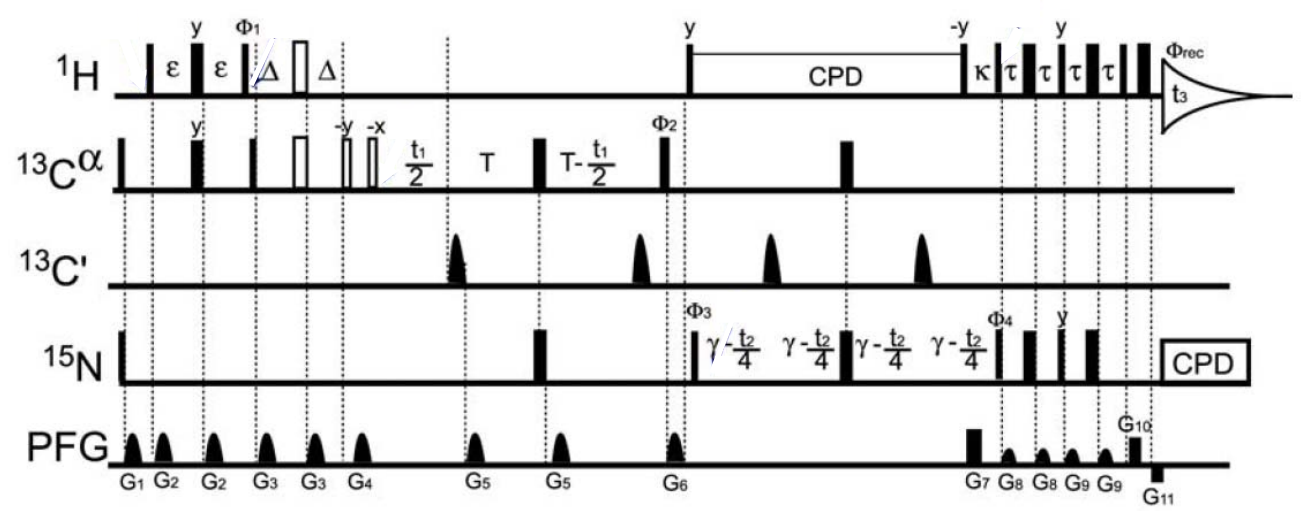

Fig. 2- 5. Pulse sequence of IPAP-(HA)CANH.

Narrow and wide pulses correspond to flip angles of $90^{\circ}$ and $180^{\circ}$, respectively, with phase $\mathrm{x}$ unless indicated. Spectral overlap is minimized by an IPAP selection element (open pulses).(Yang and Nagayama 1996, Ottiger et al. 1998) Pulses following the cpd ${ }^{1} \mathrm{H}$-decoupling yield gradient-enhanced ${ }^{15} \mathrm{~N} \rightarrow{ }^{1} \mathrm{HN}$ magnetization transfer.(Kay et al. 1992) ${ }^{13} \mathrm{C} \alpha$ pulses are applied with a RF strength of $10.5 \mathrm{kHz}$. Shaped 13C' pulses have the shape of the center lobe of a $(\sin x) / x$ function, and duration of $150 \mu$ s at $151 \mathrm{MHz} 13 \mathrm{C}$ frequency. Delay durations: $\varepsilon \approx 1.4 \mathrm{~ms} ; \Delta=1.7 \mathrm{~ms} ; \mathrm{T}=14 \mathrm{~ms} ; \gamma=7 \mathrm{~ms} ; \kappa=5.35 \mathrm{~ms} ; \tau=2.67 \mathrm{~ms}$. Phase cycling: $\varphi 1=\mathrm{y},-\mathrm{y} ; \varphi 2$ $=\mathrm{x} ; \varphi 3=2(\mathrm{x}), 2(-\mathrm{x}) ; \varphi r e c=\mathrm{x}, 2(-\mathrm{x}), \mathrm{x}$. Quadrature detection in $\mathrm{t} 1$ and $\mathrm{t} 2$ is obtained by States-TPPI on $\varphi 2$ and by Rance-Kay alternation(Kay et al. 1992) on gradient(G7)/phase( $\varphi 4)$, respectively. All gradients are sine-bell shaped, with $25 \mathrm{G} / \mathrm{cm}(15 \mathrm{G} / \mathrm{cm}$ for $\mathrm{G} 3 ; 10 \mathrm{G} / \mathrm{cm}$ for $\mathrm{G} 7,10,11)$ at their center. Durations: $\mathrm{G} 1,2,3,4,5,6,7,8,9,10,11=1.5,1.1,0.75,1.5,1.1,2.0,2.075,1.2,1.1,0.2,0.075 \mathrm{~ms}$, with $\mathrm{z}$ gradient axis. (adapted and modified from Zweckstetter and Bax, 2001(Zweckstetter and Bax 2001))

\subsubsection{Other spectroscopic methods}

\subsubsection{UV absorbance}

The concentration of proteins in solution was determined by absorption at $280 \mathrm{~nm}\left(\mathrm{~A}_{280}\right)$ and molar extinction coefficient at given wavelength $\left(\varepsilon_{280}\right)$.

$$
A_{280}=\varepsilon_{280} \cdot c \cdot d
$$

where $\quad \mathrm{A}_{280}: \quad$ absorption at $280 \mathrm{~nm}$

$\varepsilon_{280}$ : molar extinction coefficient at $280 \mathrm{~nm}\left(\mathrm{M}^{-1} \mathrm{~cm}^{-1}\right)$

c: $\quad$ protein concentration $(\mathrm{M})$ 


\section{d: $\quad$ path length of light $(\mathrm{cm})$}

\subsubsection{Circular dichroism (CD)}

CD spectra were recorded using Jasco-720 CD spectrometer in the department of molecular biology, MPIBPC. $200 \mu \mathrm{L}$ of $20 \sim 30 \mu \mathrm{M}$ sample in a $0.1 \mathrm{~cm}$ path length cuvette was used. The spectra were acquired from $190 \mathrm{~nm}$ to $250 \mathrm{~nm}$ with $1 \mathrm{~nm}$ resolution at a scan-rate $20 \mathrm{~nm} / \mathrm{min}$. The spectra were averaged for the triplicates. The mean residue ellipticity was calculated automatically by the spectrometer software.

The CD spectrum analysis for the protein secondary structure is performed with DICHROWEB server (Whitmore and Wallace 2004).

\subsubsection{Dynamic light scattering (DLS)}

DLS was performed with Wyatt DynaPro Titan equipped with temperature controller. 12 $\mu \mathrm{L}$ of sample with concentration of $\sim 100 \mu \mathrm{M}$ was used. All the samples were filtered with $0.2 \mu \mathrm{m}$ syringe filter before the mesaurement. The hydrodynamic radius was calculated from the acquired translational diffusion coefficient by the software.

\subsubsection{Atomic force microscopy (AFM)}

AFM images were recorded using Asylum MFP-3D AFM machine in the department of NanoBiophotonics, MPIBPC. Impurities on glass surface were removed by plasma cleaning. $5 \sim 10 \mu \mathrm{L}$ of sample solution was deposited on the glass or freshly prepared mica surface. After drying in air for 1 2 hours, unbound sample and buffer were washed with $100 \mu \mathrm{L}$ of distilled water. 


\section{Amino acid bulkiness defines the local conformations and dynamics of natively unfolded $\alpha$-synuclein}

\subsection{Introduction}

Intrinsically unfolded proteins remain, however, beyond the reach of classical structural biology because of their inherent conformational heterogeneity. The overall properties of unfolded proteins are similar to the random coil state and are rather insensitive to the details of the amino acid sequence (Tanford et al. 1966). Spectroscopic measurements, however, suggest the presence of sequence-specific residual secondary and even tertiary structure in unfolded states of proteins (Fiebig et al. 1996, Shortle and Ackerman 2001, Klein-Seetharaman et al. 2002, Petkova et al. 2002, Schwarzinger et al. 2002, Mohana-Borges et al. 2004).

NMR is an optimal tool to study unfolded proteins at atomic resolution. Residual dipolar couplings (RDCs) are particularly sensitive probes for the structure and dynamics of biomolecules. When confined in weakly aligning media, unfolded proteins display surprisingly variable RDCs as a function of position along the chain, possibly even encoding the native topology (Shortle and Ackerman 2001, Mohana-Borges et al. 2004). This is in clear contrast to the bell-like smooth distribution of RDCs that is expected for a random flight chain (Louhivuori et al. 2003) and that was interpreted as alignment of extended or polyproline II conformations (Mohana-Borges et al. 2004).

As a recent progress to interpret the variable RDCs of unfolded proteins, it was shown that RDCs in denatured proteins can be predicted from ensembles of unfolded 
structures generated by using a self-avoiding statistical coil model, which was based on residue specific $\varphi / \psi$ propensities from loop regions of a folded protein database (Bernado et al. 2005b, Jha et al. 2005).

With the concept that self-avoiding statistical coil is governed by steric effect of each side chain, we found that a much simpler model can also explain many aspects of the profile of RDCs in unfolded proteins: Amino acid bulkiness, the ratio of the side chain volume to its length,(Zimmerman et al. 1968) predicts clearly observable features reporting on the local conformational behavior of natively unfolded proteins, such as $\alpha \mathrm{S}$ and tau fragment.

\subsection{Materials and methods}

\subsubsection{Sample preparation}

${ }^{15} \mathrm{~N}$-labeled wt $\alpha \mathrm{S}$ sample was prepared as described in Chapter 2.2. 1. 2. $100 \mu \mathrm{M} \alpha \mathrm{S}$ in $50 \mathrm{mM} \mathrm{NaPi}, 100 \mathrm{mM} \mathrm{NaCl}, \mathrm{pH} 7.4$ was used.

\subsubsection{RDC measurement}

All the procedure to measure ${ }^{1} \mathrm{D}_{\mathrm{NH}}$ of $\alpha \mathrm{S}$ is described in Chapter 2. 2. 2. 6. Briefly, $\alpha \mathrm{S}$ was weakly aligned in n-octylpenta(ethylene glycol)/octanol (Ruckert and Otting 2000), and $\mathrm{RDCs}$ were determined at $15^{\circ} \mathrm{C}$ in $20 \mathrm{mM}$ Tris $\cdot \mathrm{HCl}, \mathrm{pH} 7.5,100 \mathrm{mM} \mathrm{NaCl}$, and with $8 \mathrm{M}$ urea for denatured condition.

\subsubsection{R1 $\rho$ measurement}

The procedure to measure longitudinal relaxation rate in rotating frame $(\mathrm{R} 1$.. is 
described in Chapter 2.2.2.5. Total six 2D spectra were acquired using relaxation delays of $8,32,48,88,176$ and $8 \mathrm{~ms}$, and an on-resonance spin-lock pulse at $2.5 \mathrm{kHz}$ in order to cover the full range of $15 \mathrm{~N}$ spectra width.

\subsubsection{Calculation of bulkiness profile}

Amino acid specific bulkiness values were taken from Zimmerman et al.(Zimmerman et al. 1968). Bulkiness profile with sliding windows size of 3, 5 and 7 along with $\alpha \mathrm{S}$ sequence were generated and compared with RDCs in physiological condition and in denaturing condition. Sliding window size 5 was chosen for the further experiment.

Most deviations between bulkiness profile and RDCs existed near proline residues and both terminals. The deviations near proline residues were compensated by increasing bulkiness value for prolines by an empirical scaling factor of 1.6. Larger flexibility at the ends of the polypeptide chain was taken into account by combining the bulkiness profile with a bell-shaped curve that is based on the assumption that the influence of neighboring residues decays exponentially as the distance from a given residue (persistence length of the chain $=7$ ) (Schwalbe et al. 1997). Note that this only affects bulkiness values for residues at the termini.

\subsubsection{Flexible-meccano model generation and RDC calculation}

In the flexible-meccano approach, peptide chains are built using randomly selected $\varphi / \psi$ pairs drawn from a database of amino acid specific conformations present in loop regions of high-resolution X-ray structures (Bernado et al. 2005b).

The alignment tensor was predicted for each conformer on the basis of the threedimensional shape using PALES (Zweckstetter and Bax 2000), and associated RDCs 
are calculated for each $\mathrm{NH}$ vector with respect to this tensor. RDCs from each site are then averaged over 50,000 conformers to ensure convergence.

\subsection{Results and discussion}

\subsubsection{Experimental RDCs and bulkiness of $\alpha \mathrm{S}$}

Using RDCs and paramagnetic relaxation enhancement from specifically attached paramagnetic nitroxide radicals, it was shown that despite its high flexibility, native $\alpha \mathrm{S}$ adopts an ensemble of conformations that are stabilized by long-range interactions (Bertoncini et al. 2005b). The ${ }^{1} \mathrm{D}_{\mathrm{NH}} \mathrm{RDC}$ profile of $\alpha \mathrm{S}$, therefore, reflects long-range interactions. In Fig. 3- 1, RDCs observed from native $\alpha \mathrm{S}$ and denatured $\alpha \mathrm{S}$ are compared with the amino acid bulkiness. The refined bulkiness profile closely matches the variation of RDCs observed in $\alpha \mathrm{S}$ as a function of position along the chain. In regions, in which large RDCs were observed, many residues with bulky side chains are present (Fig. 3-1A, B).

The regions with large RDCs are separated by residues that showed couplings close to zero. In these linker sequences mainly amino acids with small side chains such as glycine and alanine are found: ${ }^{29} \mathrm{AAG}^{31},{ }^{67} \mathrm{GGA}^{69},{ }^{84} \mathrm{GAGS}^{87},{ }^{106} \mathrm{GA}^{107}$. The largest deviations between the RDC pattern and the bulkiness profile were present in the Nterminus and for residues 115-119 and 125-129.

Upon addition of urea, these deviations were removed (Fig. 3- 1C). Although the interaction between the $\mathrm{N}$ - and the C-terminus is expected to be mostly electrostatic, the C-terminus forms hydrophobic interactions with the central part of $\alpha \mathrm{S}$. Thus, the observed changes in RDCs suggest a complex network of long-range interactions, 
giving rise to a more complex RDC base-line upon which local structure is superimposed (Bernado et al. 2005a, Bertoncini et al. 2005b).
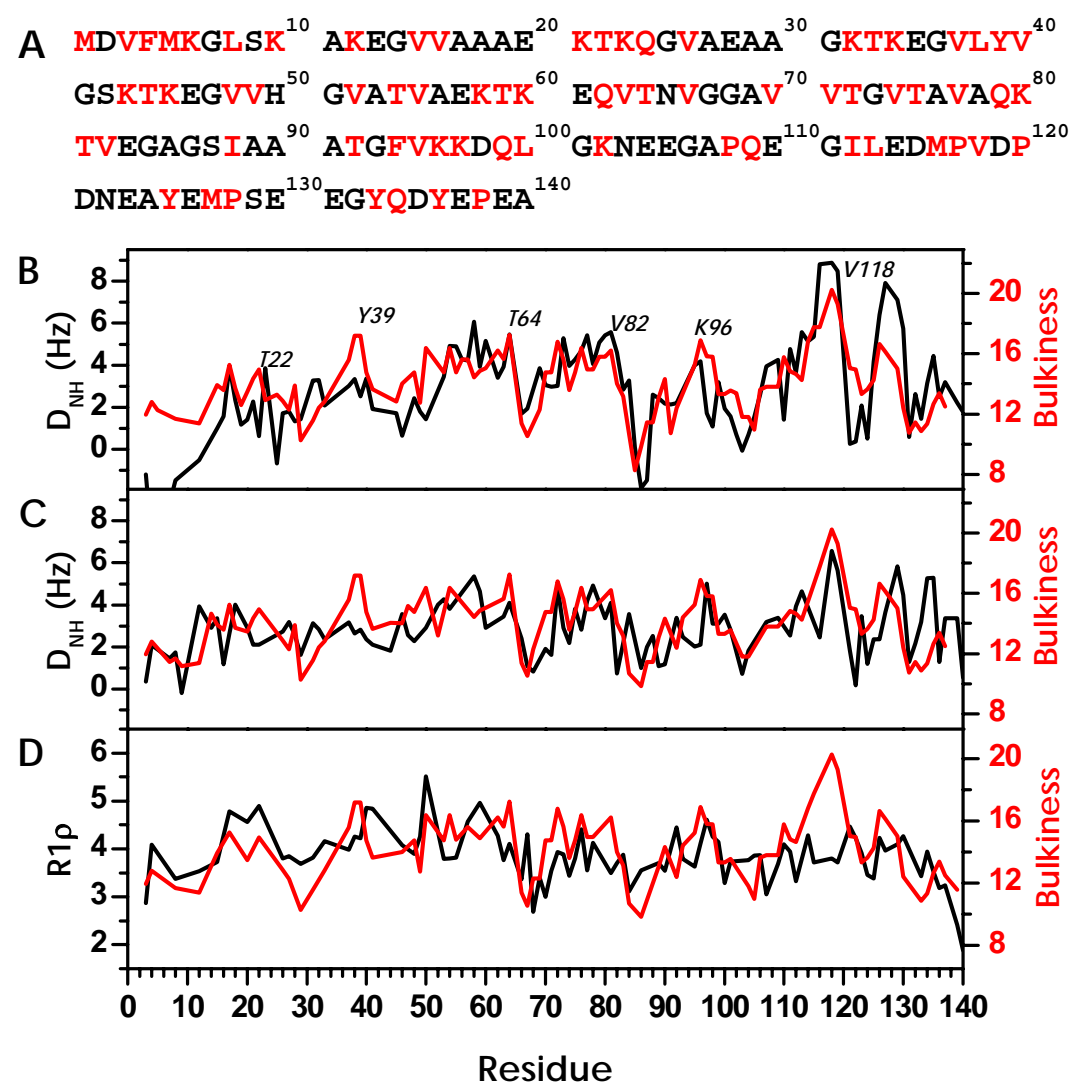

Fig. 3- 1. Bulkiness profile and NMR dynamics parameters of $\alpha \mathrm{S}$.

(A) Amino acid sequence of $\alpha \mathrm{S}$. Bulky amino acids bulkiness $>14$ ) are marked in red. (B, C) Comparison of amino acid bulkiness (five residue window average) (red) with one-bond ${ }^{1} \mathrm{H}^{15}{ }^{15}$ RDCs (black) observed in $\alpha \mathrm{S}$ (B) in buffer and (C) in buffer with $8 \mathrm{M}$ urea. (D) Comparison of amino acid bulkiness (red) with $15 \mathrm{~N}$ R1 $\rho$ rates (black). Bulkiness values are only shown for residues with experimental data.

Whereas RDCs probe both structure and dynamics, heteronuclear relaxation rates monitor directly backbone motional restrictions. In particular, on-resonance ${ }^{15} \mathrm{~N} \mathrm{R}_{1 \rho}$ transverse relaxation rates report on motions that occur on the pico-to-nanosecond and micro-to-millisecond time scale. $\mathrm{R}_{1 \rho}$ values vary along the chain of $\alpha \mathrm{S}$ in a similar way as is seen for RDCs and the bulkiness profile closely matches the $\mathrm{R}_{1 \rho}$ pattern (Fig. 3- 
1D). This indicates that the local steric interactions between side chains and the backbone restrict motions on the pico-to-nanosecond and micro-to-millisecond time scale, in agreement with relaxation time measurements previously reported for acidunfolded apomyoglobin (Schwarzinger et al. 2002). The largest deviation between the $\mathrm{R}_{1 \rho}$ and the bulkiness profile was observed in the vicinity of P117 and P120, suggesting that prolines also restrict slower motions for which $\mathrm{R}_{1 \rho}$ rates are not sensitive but ${ }^{1} \mathrm{D}_{\mathrm{NH}}$ could detect.

The minimum deviation between experimental RDCs and the bulkiness pattern was obtained for a five $(i-2, \ldots, i+2)$ to seven $(i-3, i-2, \ldots, i+2, i+3)$ residue window average. Averaging over several residues simulates the neighbouring residues effect exerts on the local conformation and dynamics of each amino acid in a polypeptide chain. This strongly suggests that the Flory isolated-pair hypothesis (Flory 1969), which considers only the nearest-neighboring residue $(i-1, i, i+1)$, is not sufficient to explain RDCs in natively unfolded $\alpha \mathrm{S}$. A window size of five to seven is in agreement with other measurements (Schwalbe et al. 1997, Schwarzinger et al. 2002, Mohana-Borges et al. 2004) and calculations (Ohkubo and Brooks 2003, Tran and Pappu 2006) that estimated the length scale over which spatial correlations decay in denatured proteins to range from six to nine residues.

The effect of proline scaling and a bell-shape smoothing curve for terminal flexibility of polypeptide chain is observable by comparison Fig. 3- 1 and Fig. 3- 2. 


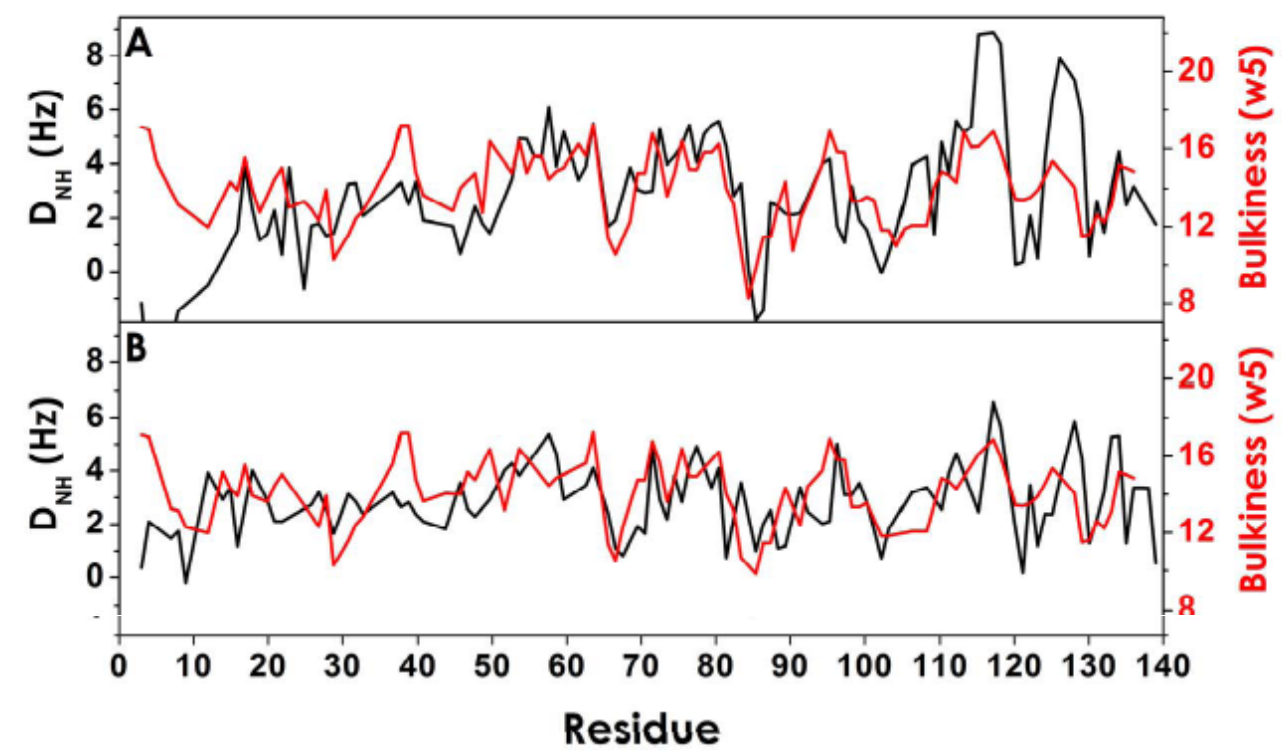

Fig. 3- 2. Comparison of the raw bulkiness profile (red) with one-bond RDCs (black) of $\alpha \mathrm{S}$.

In buffer only (A), and in buffer with $8 \mathrm{M}$ urea (B). No correction for the bulkiness values of prolines or for the increased flexibility at the ends of the chain was applied. The improved correlation between the bulkiness and ${ }^{1} \mathrm{D}_{\mathrm{NH}}$ is due to the loss of the long-range interaction in the native $\alpha \mathrm{S}$ by urea.

\subsubsection{Ensemble-simulated RDCs and bulkiness}

The unfolded protein ensemble generated by flexible-meccano could reproduce the experimental RDCs (Bernado et al. 2005b). The same approach was applied to $\alpha \mathrm{S}$; however, RDCs in N- and C-terminus were not reproduced from 50,000 structures. The experimental RDCs of wt $\alpha \mathrm{S}$ were reproduced when a part of conformations were chosen based on the distance between regions (Bernado et al. 2005a). Considering 50,000 conformers can represent the unfolded state of a protein, RDCs over these conformers were calculated and compared with the bulkiness profile.

Fig. 3- 4 shows the comparison of RDCs predicted from the flexible meccano ensemble with the pattern of side chain bulkiness. A highly similar variation of values is observed along the polypeptide chain of $\alpha \mathrm{S}$. Slight deviations were observed in the vicinity of residue $\mathrm{Y} 39$, for residues $65-70,85-90$ and $125-130$, and at the C-terminus of 
$\alpha \mathrm{S}$, mostly regions in which glycine or proline residues are present. The similarity between these profiles provides a direct experimental proof for the dominating influence of steric interactions on the composition of the Ramachandran plot. RDCs are reproduced equally well by sampling only residue-specific $\varphi / \psi$ distributions or by only considering the bulkiness of amino acid side chains.

Fig. 3- 3. Representative conformations generated by flexible-meccano.

100 of 50,000 generated structures were shown with a conformer from Bertoncini et al.(Bertoncini et al. 2005b).

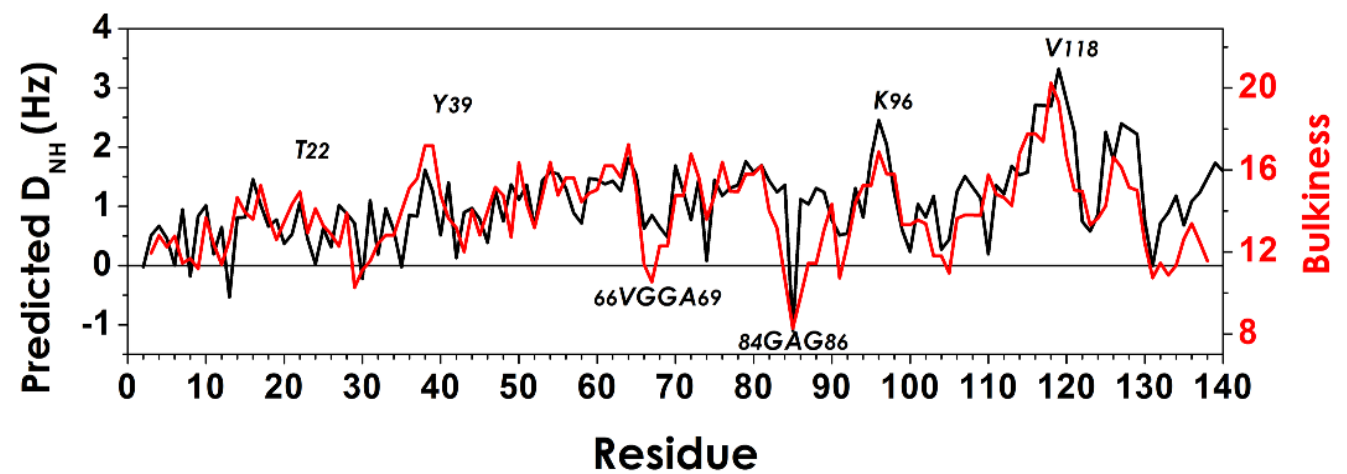

Fig. 3- 4. Comparison of amino acid bulkiness (red) with ensemble-averaged RDCs (black). 
RDCs were predicted from 50,000 coil structures generated by flexible-meccano.

Removing steric exclusion from the flexible meccano approach does not affect the results of the simulation very strongly (Fig. 3- 5). In addition, explicit inclusion of nearest neighbor interactions into flexible-meccano was not required. On the other hand, Jha et al. concluded that the identity of neighboring residues needs to be incorporated to improve RDC reproduction (Jha et al. 2005). The origin of this disagreement about neighboring residue effect is not clear.

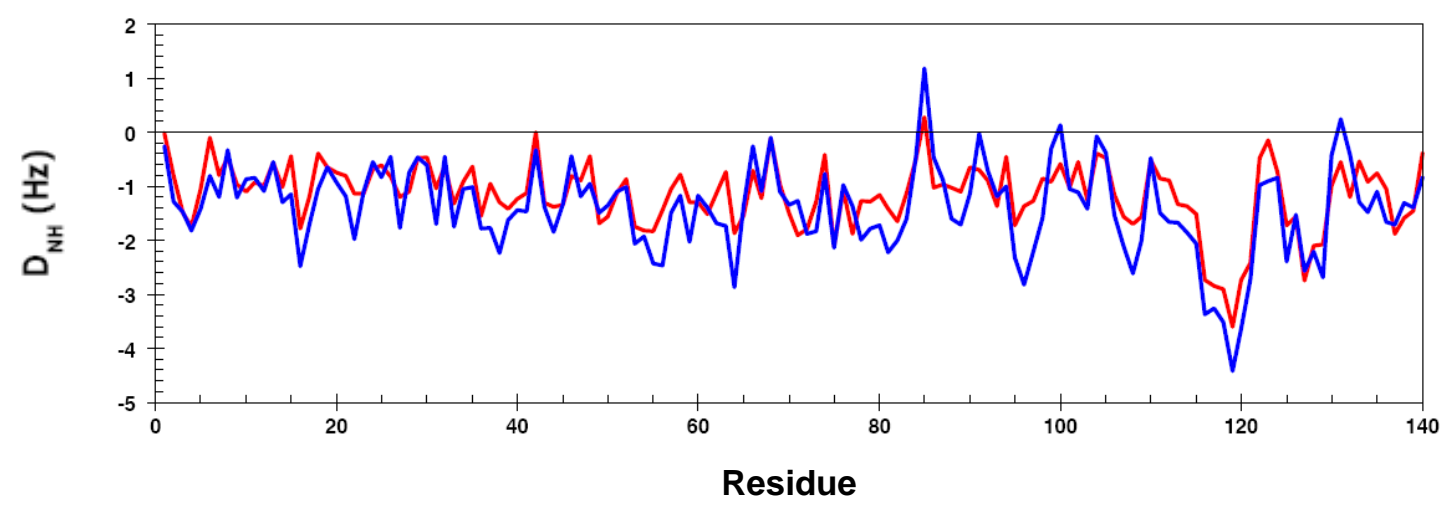

Fig. 3- 5. Comparison of RDCs predicted with/ without steric obstruction.

RDCs were predicted from 50,000 coil structures of $\alpha \mathrm{S}$ generated by flexible-meccano using amino-acid specific conformational propensities with steric obstruction (blue), without steric obstruction (red).

\subsubsection{Bulkiness and RDCs in denatured protein}

We extended our study to other protein systems. Previously, RDCs observed in the chemical denatured SNase fragment, $\Delta 131 \Delta$, were used to argue for the presence of a native-like organization of chain segments in unfolded proteins (Shortle and Ackerman 2001). Comparison of the experimental RDCs of $\Delta 131 \Delta$ with side chain bulkiness averaged over a five residue window, however, suggests that the variation of RDCs 
along the chain of $\Delta 131 \Delta$ can be explained without the need for invoking a native-like topology (Fig. 3- 6A).Similarly, the RDC pattern observed in chemically denatured eglin C (Ohnishi et al. 2004) (Fig. 3- 6B) and in a 130-residue fragment of natively unfolded tau closely matches the bulkiness profile (Fig. 3- 7).

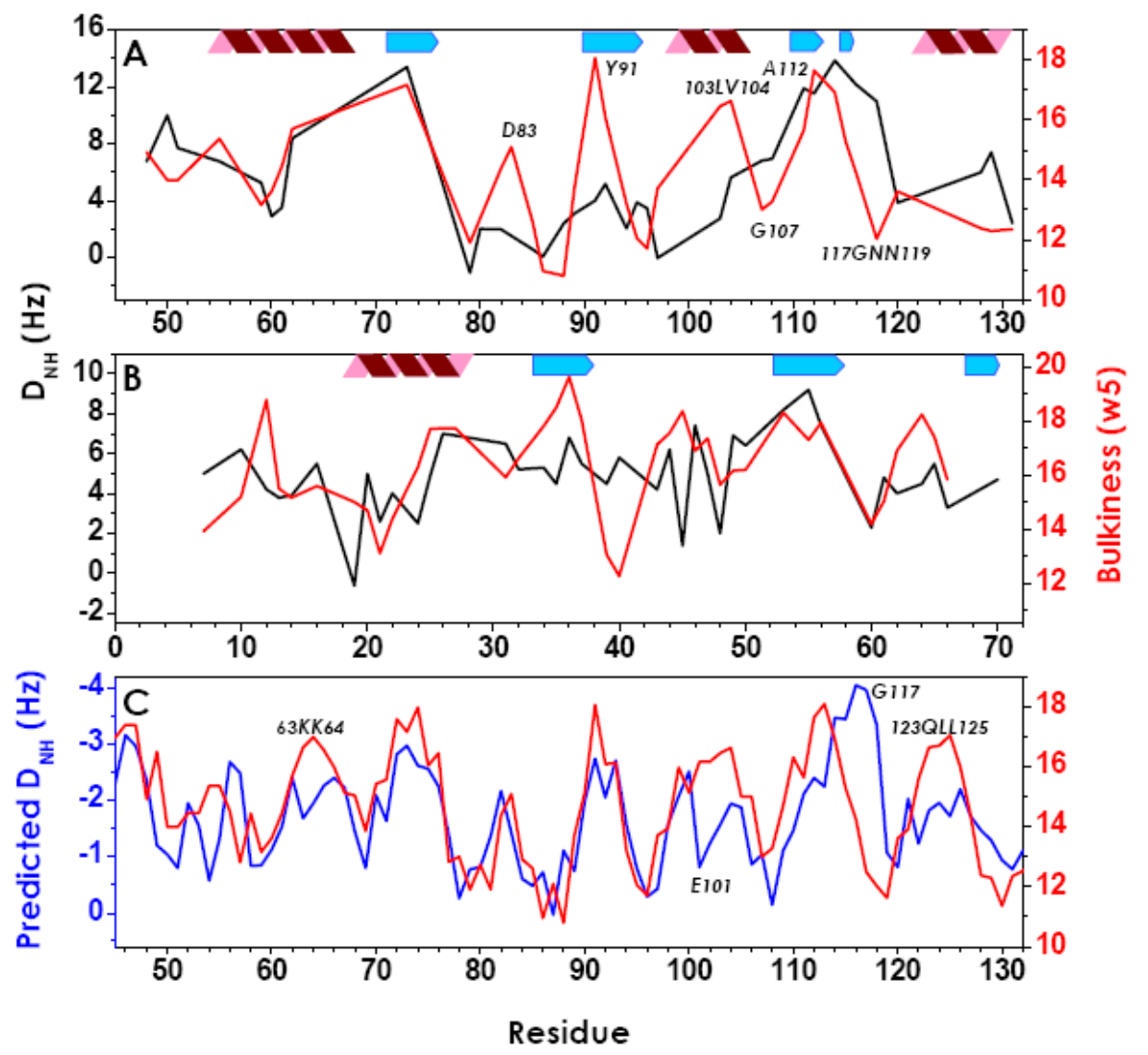

Fig. 3- 6. Comparison of bulkiness and ${ }^{1} D_{\mathrm{NH}}$ of unfolded proteins.

Bulkiness profile (red) is compared with one-bond ${ }^{1} \mathrm{D}_{\mathrm{NH}}$ RDCs (black) observed (A) in the chemically denatured fragment $\Delta 131 \Delta$ and (B) in chemically denatured eglin C. (C) Comparison of amino acid bulkiness (five residue window average; red) with RDCs predicted from 50,000 coil structures of $\Delta 131 \Delta$ generated by flexible-meccano (blue). Regions of regular secondary structure in the folded state of SNase and eglin C are marked. In (A) and (B) bulkiness values are only shown for residues, for which experimental data were available. 


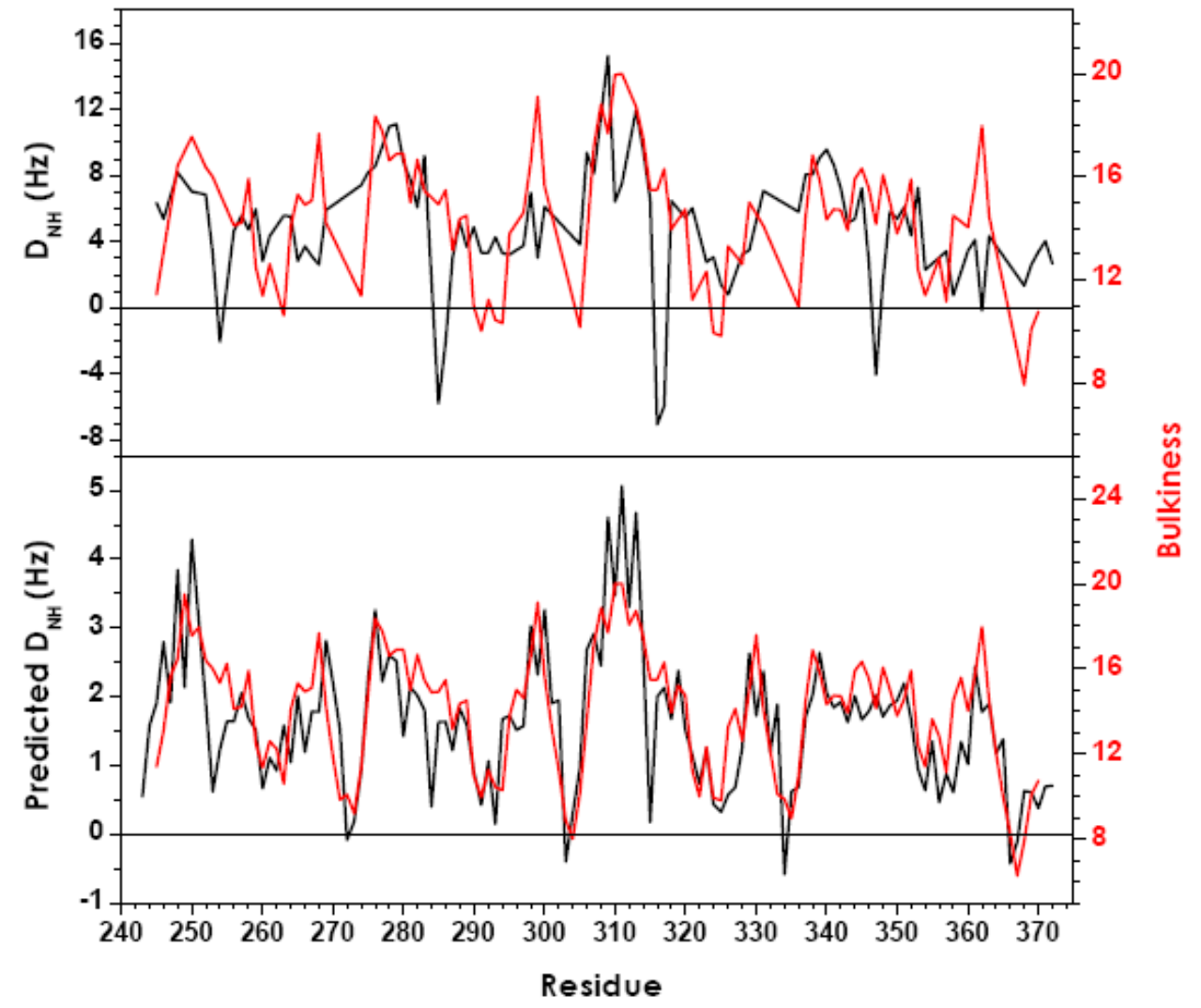

Fig. 3- 7. Comparison of bulkiness and ${ }^{1} \mathrm{D}_{\mathrm{NH}}$ of $\mathrm{K} 18$.

(A) Comparison of the bulkiness of amino acids (red) with one-bond ${ }^{1} \mathrm{D}_{\mathrm{NH}} \mathrm{RDCs}$ (black) observed in a 130-residue fragment (K18) of the natively unfolded tau protein.(Mukrasch et al. 2007) Bulkiness values are only shown for residues, for which experimental data were available. (B) Comparison of amino acid bulkiness (five residue window average; red) with RDCs predicted from 50,000 coil structures of K18 generated by flexible-meccano (black). The negative experimental RDCs visible in (A) are due to the presence of local turns (Mukrasch et al. 2007).

Thus, a more likely explanation for the variation of RDCs along polypeptide chains is that minimization of steric overlap promotes chain stretching. In more extended parts of the chain, the interaction direction between the dipolar orientations tends to be perpendicular to the external field resulting in increased RDCs. In addition, more extended backbone conformations are expected to align more effectively, further increasing the magnitude of RDCs in these regions. 


\subsubsection{Comparison of bulkiness with other physico-chemical properties}

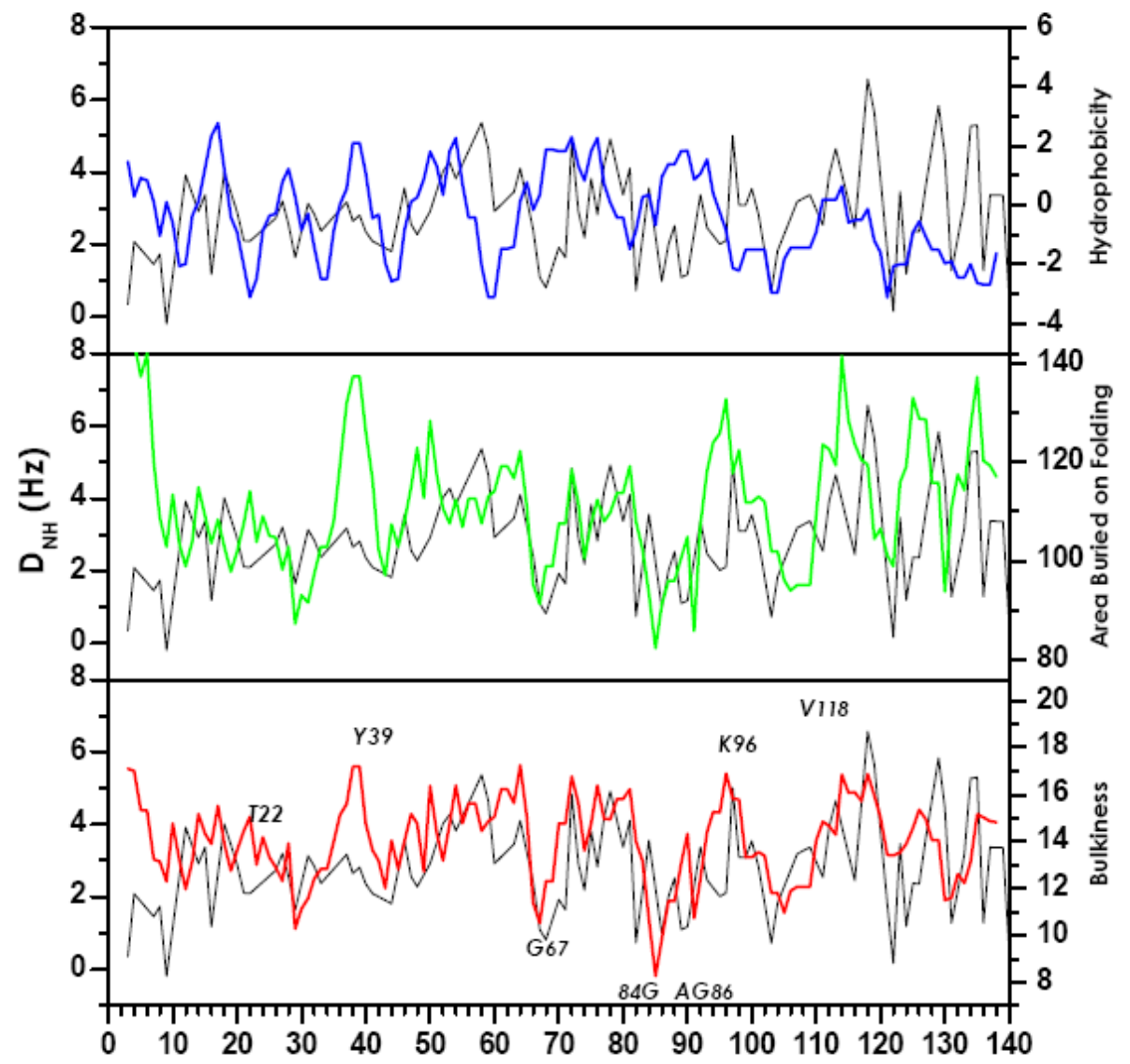

Fig. 3- 8. Comparison of residue specific physico-chemical properties.

Comparison of the hydrophobicity (Kyte and Doolittle 1982) (blue), the area buried upon folding (Rose et al. 1985) (green) and the bulkiness of amino acids (red) with one-bond ${ }^{1} \mathrm{D}_{\mathrm{NH}} \mathrm{RDCs}$ (black) of $\alpha \mathrm{S}$ in $8 \mathrm{M}$ urea condition. In each case, only the average over five-residue window is applied.

In this study, bulkiness of amino acid is revealed as a determinant of local structure and dynamics. There are several other properties of amino acid, which are thought to be responsible for the protein folding; hydrophobicity (Kyte and Doolittle 1982) and the area upon folding (Rose et al. 1985). To verify a better property that is able to explain the local dynamics, bulkiness is compared with hydrophobicity and the area upon 
folding in Fig. 3- 8. All three properties are plotted as a function of residue number with five residue window average, and compared with ${ }^{1} \mathrm{D}_{\mathrm{NH}}$ RDCs of $\alpha \mathrm{S}$ in $8 \mathrm{M}$ urea condition.

The correlation of the hydrophobicity profile with the experimentally observed RDCs is significantly worse than the RDC-bulkiness correlation. However, the profile of the "area buried upon folding" of $\alpha \mathrm{S}$ is very similar to the pattern of experimental RDCs as well as the bulkiness profile. For residues 40-50, 92-97 and 110-120 the bulkiness profile fits slightly better to the experimental RDCs.

Compared to the "area buried upon folding," however, the bulkiness of side chains is one of the most basic properties of amino acids, which defines the steric hindrance in the Ramachandran plot. Considering that the flexible-meccano predicted RDCs (which closely resemble the bulkiness profile) are based on the distribution of amino acids in the Ramachandran plot and reproduce RDCs of unfolded protein with no secondary structure or long-range interaction, the amino acid bulkiness is the property defining the local conformations and dynamics of unfolded proteins.

\subsection{Summary and Conclusion}

Our results demonstrate that, although various types of intramolecular interactions such as electrostatic interactions and solvent interactions, play important roles, simple considerations of the bulkiness of amino acids predicts a major component of diverse parameters dependent on the local conformation and dynamics of $\alpha \mathrm{S}$ and other natively unfolded proteins.

Deviations from this random coil behavior, as evidenced by RDCs in the N- and C- 
terminal domain of $\alpha \mathrm{S}$, and those in the turn conformation of tau protein fragment, can provide insight into residual secondary structure and long-range transient interactions in weakly structured proteins. The local steric restrictions in the unfolded state can also bias the conformational search toward native-like elements and thereby promote funnellike energy landscapes in protein folding (Dill and Chan 1997). 


\section{Aggregation prone conformation of $\alpha$-synuclein at low pH}

\subsection{I ntroduction}

In protein aggregation, monomeric proteins, globular or unstructured, are converted into amyloid fibril through intermediate states (Ross and Poirier 2004, Eisenberg et al. 2006). The structural information in each step should be valuable for understanding the transition mechanism closely linked to the pathology of a related disease. $\alpha$-synuclein $(\alpha S)$, main component in Lewy body found in the brain of Parkinson's disease (PD), has been investigated intensively, and the atomic resolution structures of $\alpha \mathrm{S}$ monomer in physiological solution or on the artificial membrane were determined by several biophysical and computational methods (Lee et al. 2004, Bernado et al. 2005a, Bertoncini et al. 2005b, Ulmer et al. 2005, Sung and Eliezer 2006, Uversky 2007, Uversky et al. 2008, Wu et al. 2008), and the fibrillar state structure (Heise et al. 2005, Chen et al. 2007, Vilar et al. 2008) also have been studied to elucidate the structural information. While increasing structural information is available for the $\alpha \mathrm{S}$ monomer and the fibrillar state, the intermediate or oligomer structures, however, are determined only at low resolution (Uversky et al. 2001a, Lashuel et al. 2002, Kaylor et al. 2005, Apetri et al. 2006).

The aggregation kinetics of $\alpha \mathrm{S}$, in vitro, is affected by several factors like low $\mathrm{pH}$, molecular crowding reagents, high temperature, high salt concentration, polyamines, ethanol, metals, Heparin, and pesticide (Uversky 2007). In such conditions, aS may adopt aggregation-prone or intermediate conformation, and reveal critical factors 
responsible for the aggregation. Uversky et al. showed that $\alpha \mathrm{S}$ adopts a partial folded monomer at low $\mathrm{pH}(\mathrm{pH} 3)$ and high temperature $\left(95^{\circ} \mathrm{C}\right)$ based on ANS fluorescence and CD spectroscopy (Uversky et al. 2001b). In order to obtain the structural information of partially folded $\alpha \mathrm{S}$ monomer in atomic resolution, we investigated $\alpha \mathrm{S}$ conformation at low $\mathrm{pH}$ with circular dichroism (CD) and NMR spectroscopy. Several NMR methods enables us to determine $\alpha \mathrm{S}$ conformation in atomic resolution; the secondary structure propensity, rigidity of backbone chain by residual dipolar couplings (RDCs), long range interactions by paramagnetic relaxation enhancement (PRE), and hydrodynamic radius $\left(\mathrm{R}_{\mathrm{h}}\right)$ by the translational diffusion coefficient measured by PFGNMR.

\subsection{Materials and methods}

\subsubsection{Sample preparation}

${ }^{15} \mathrm{~N}$-labeled or ${ }^{13} \mathrm{C} /{ }^{15} \mathrm{~N}$-labeled $w t \alpha \mathrm{S}$ and $w t \alpha \mathrm{S}(1-108)$ samples were prepared as described in Chapter 2.2.1.2. Standard solid-phase peptide synthesis was employed to produce peptides comprising residues 105 to 136 of $w t \alpha \mathrm{S}$. The peptides were purified by reverse phase HPLC and the purity ( $>95 \%)$ was analyzed by mass spectrometry. Samples were prepared in $20 \mathrm{mM} \mathrm{Na}$ acetate, $100 \mathrm{mM} \mathrm{NaCl}, \mathrm{pH} 3$ for NMR experiment.

\subsubsection{CD experiment}

CD spectra of wt $\alpha \mathrm{S}(1-108)$ and C-terminal peptide (105-136) were recorded using Jasco-720 CD spectrometer in the department of molecular biology, MPIBPC. All the spectra were taken at room temperature $\left(\sim 25^{\circ} \mathrm{C}\right) .200 \mu \mathrm{L}$ of $20 \sim 30 \mu \mathrm{M}$ sample in a 0.1 
cm path length cuvette was used. The spectra were acquired from $190 \mathrm{~nm}$ to $250 \mathrm{~nm}$ with $1 \mathrm{~nm}$ resolution at a scan-rate $20 \mathrm{~nm} / \mathrm{min}$. and averaged over the triplicates. The mean residue ellipticity was calculated automatically by the spectrometer software. DICHROWEB (Whitmore and Wallace 2004) is used for the protein secondary structure analysis.

\subsubsection{NMR experiment}

Stepwise $\mathrm{pH}$ titration was done by Dr. Carlos W. Bertoncini in order to follow each peak position in ${ }^{1} \mathrm{H}-{ }^{15} \mathrm{~N}$ HSQC spectra. Backbone resonance assignment was performed with IPAP-(HA)CANH, HNCO experiments and the assigned ${ }^{1} \mathrm{H}$ and ${ }^{15} \mathrm{~N}$ resonances. $\mathrm{C}_{\alpha}$ and $\mathrm{C}^{\prime}$ chemical shift were used for secondary structure determination. PG-SLED pulse sequence with internal standard was used to determine hydrodynamic radius $\left(\mathrm{R}_{\mathrm{h}}\right)$ of full length wt $\alpha \mathrm{S}, \alpha \mathrm{S}(1-108)$ and C-terminal peptide (105-136).

\subsection{Results and discussion}

\subsubsection{Secondary chemical shifts of $\alpha \mathrm{S}$ at $\mathrm{pH} 3$}

In the previous study, $\mathrm{CD}$ spectrum of $w t \alpha \mathrm{S}$ at $\mathrm{pH} 3$ showed slight increase of $\beta$ strand secondary structure (Uversky et al. 2001b). Since NMR spectroscopy can detect the structural element in atomic resolution, we used NMR spectroscopy to detect the residue-specific secondary structure. In Fig. 4- 1, the backbone amide signals in ${ }^{1} \mathrm{H}_{-}{ }^{15} \mathrm{~N}$ 2D HSQC spectra of wt $\alpha \mathrm{S}$ at $\mathrm{pH} 3$ has similar cross-peak pattern with the HSQC spectrum at $\mathrm{pH} 7.4$; it shows all the cross peaks in a narrow proton spectral width $(\sim 1.2$ ppm) representing an typical unstructured protein conformation. 


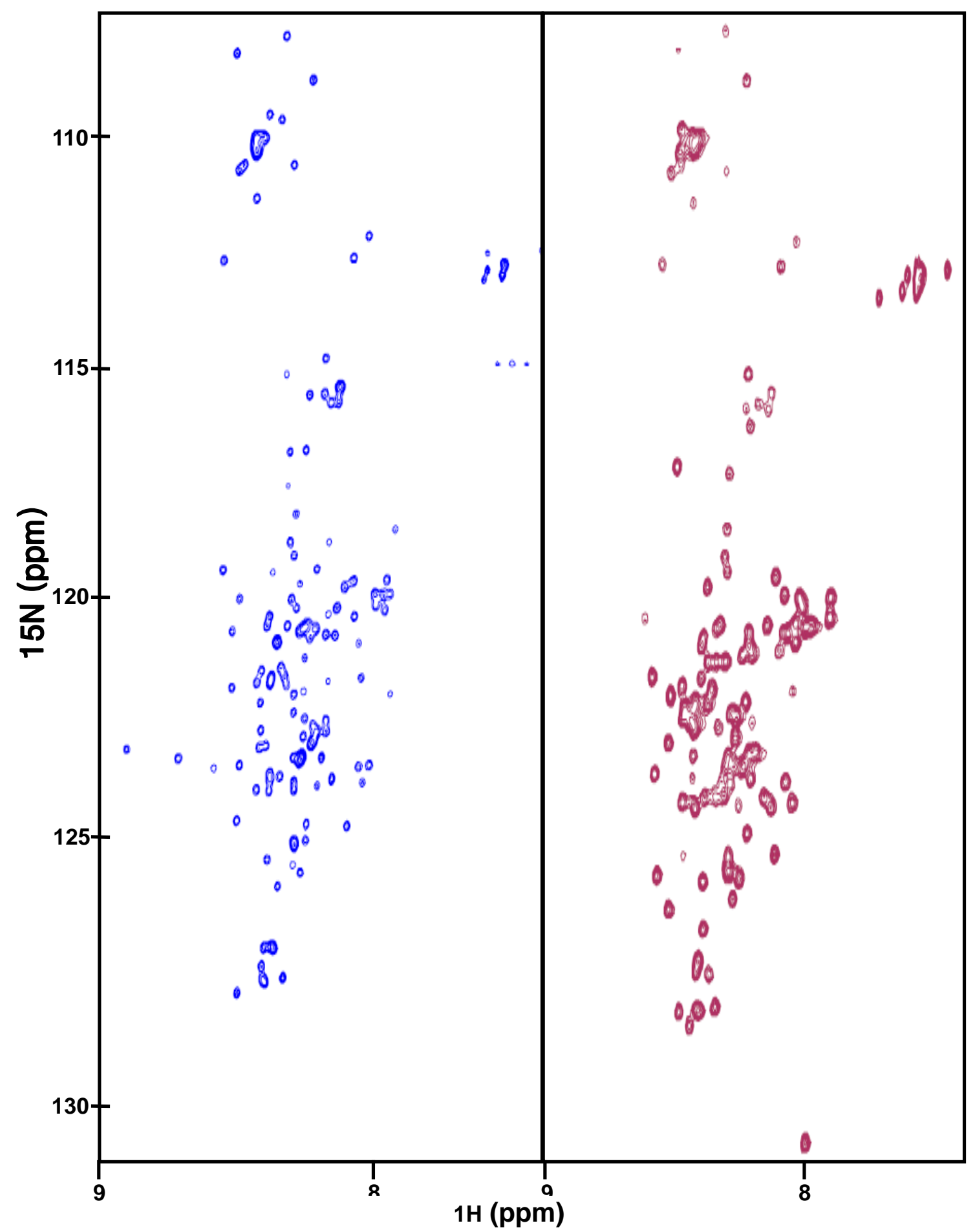

Fig. 4- 1. Comparison of ${ }^{1} \mathrm{H},{ }^{15} \mathrm{~N}$ - HSQC spectra of $\alpha \mathrm{S}$ at $\mathrm{pH}$ 3(blue) and $\mathrm{pH} 7.4$ (dark red).

Both spectra show a typical cross-peak pattern of an unfolded protein. The assignment of $\alpha \mathrm{S}$ at $\mathrm{pH} 3$ was done with $\mathrm{HNCACB}, \mathrm{CBCA}(\mathrm{CO}) \mathrm{NH}$ spectra combined with chemical shift changes in ${ }^{1} \mathrm{H}-{ }^{15} \mathrm{~N}$ HSQC spectra along with $\mathrm{pH}$ titration. 
Since ${ }^{15} \mathrm{~N}$ chemical shifts in peptide bond is not only sensitive to the secondary structure, but to chemical environment like $\mathrm{pH}$, temperature and salt, ${ }^{13} \mathrm{C}$ based backbone chemical shifts were needed to determine secondary structure propensity in $\alpha \mathrm{S}$ at $\mathrm{pH}$ 3. In order to obtain the residue-specific secondary structure propensity of $\alpha \mathrm{S}$, ${ }^{13} \mathrm{C}_{\alpha}$ chemical shifts were determined with IPAP-(HA)CANH and the secondary chemical shifts were calculated with (Eq. 1- 1). The obtained $C_{\alpha}$ secondary chemical shifts at $\mathrm{pH} 3$ are compared with those at $\mathrm{pH} 7.4$ (Fig. 4- 2). Due to the different $\mathrm{pH}$, two types of random coil values were used; 1) from statistical random coil library (Wishart and Sykes 1994, Wishart et al. 1995) with sequence dependant modification (Schwarzinger et al. 2001), and 2) from a model peptide in acidic $8 \mathrm{M}$ urea solution (Schwarzinger et al. 2000) with sequence dependant modification (Schwarzinger et al. 2001). The former was used for the secondary chemical shifts calculation of $\alpha \mathrm{S}$ at $\mathrm{pH}$ 7.4 in a previous study (Bussell and Eliezer 2001); the latter is more appropriate for low $\mathrm{pH}$ condition. Considering, $\mathrm{pH}$ dependence of the random coil values, one can compare the open bars in the upper panel and the colored bars in the lower panel to estimate the relative secondary structure propensities of $\alpha \mathrm{S}$ at both $\mathrm{pH} 7.4$ and $\mathrm{pH} 3$.

Although the overall profile of $\alpha \mathrm{S}$ at $\mathrm{pH} 3$ along the sequence has too weak deviation from the random coil to define a stable secondary structure, the secondary chemical shifts in near-NAC region (40 95) have more negative values compared to those from $\mathrm{pH} 7.4$, which corresponds to more $\beta$-sheet propensity at $\mathrm{pH} 3$. Due to the ensemble averaged character of unfolded protein, the secondary chemical shifts obtained at $\mathrm{pH} 3$ implies that more conformers adopt $\beta$-sheet structure in near-NAC region that serves as a core region in fibrillar state. 

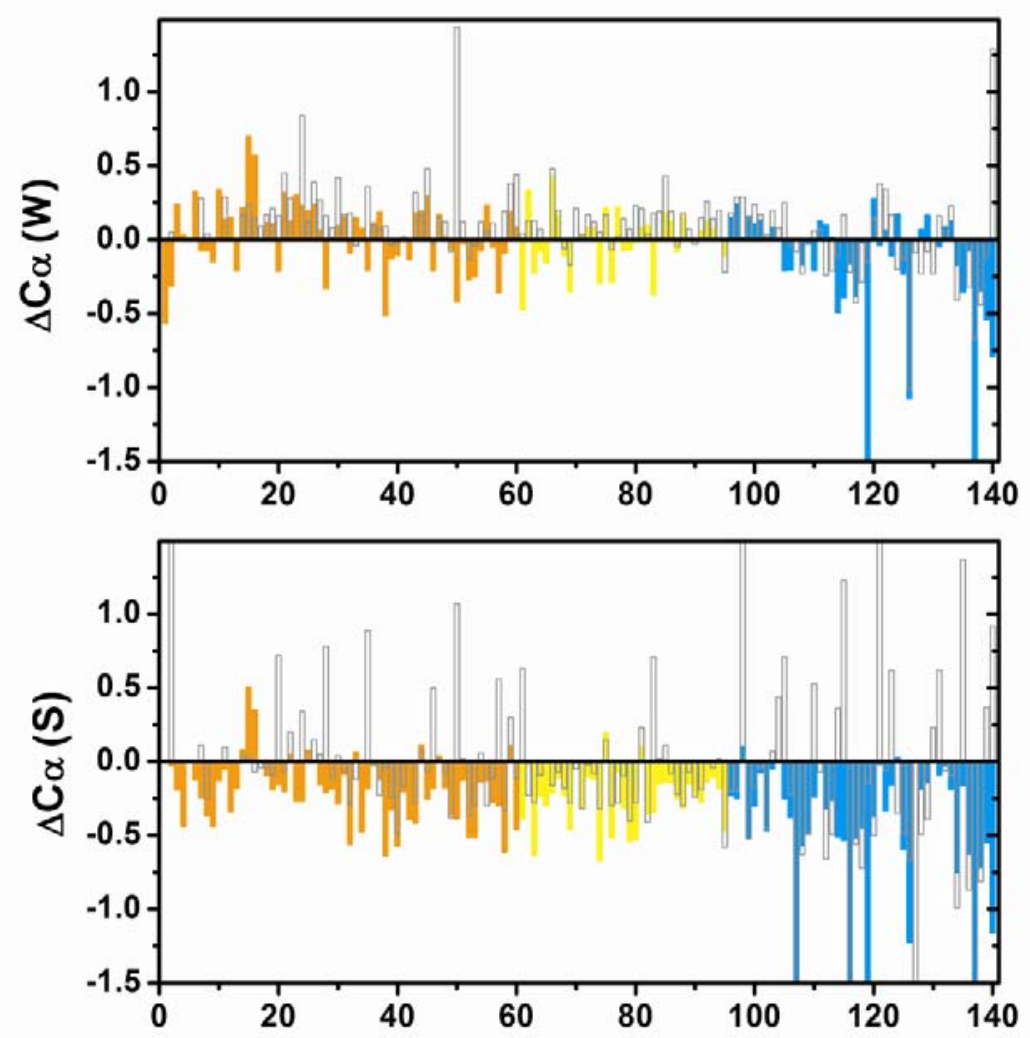

Fig. 4- 2. Secondary chemical shifts of $\alpha \mathrm{S}$ at $\mathrm{pH} 3$ compared with pH 7.4 values.

The $\mathrm{C}_{\alpha}$ secondary chemical shifts of $\alpha \mathrm{S}$ at $\mathrm{pH} 3$ (colored bar) are more negative that those of $\mathrm{pH} 7.4$ (open bar), which corresponds to more $\beta$-sheet propensity at $\mathrm{pH} 3$. Random coil values from Wishart et al. (Wishart and Sykes 1994, Wishart et al. 1995) are used with sequence dependant correction from Schwarzinger et al. (Schwarzinger et al. 2001) for the top panel, and those from Schwarzinger et al. (Schwarzinger et al. 2000, Schwarzinger et al. 2001) are used for the bottom panel..

\subsubsection{PRE and hydrodynamic radius revealed collapsed C-terminus}

Paramagnetic relaxation enhancement (PRE) has shown the evidences of long-range contact in many unfolded proteins. MTSL spin label was introduced into three single Cys mutants of wt $\alpha \mathrm{S}$ in position of $\mathrm{A} 18, \mathrm{~A} 90$, and $\mathrm{A} 140$, which were used for the previous studies (Bertoncini et al. 2005a, Bertoncini et al. 2005b).

The overall profile showed several differences from that of $\mathrm{pH}$ 7.4. When the spin label is attached to position A18C, the attenuation occurs only nearby residues, which 
reveals that the long range interactions between $\mathrm{N}$ - and $\mathrm{C}$-terminus, which had been observed at pH 7.4, disappear (left panel in Fig. 4- 3). In case of A90C $\alpha \mathrm{S}$, the signals from C-terminus are significantly attenuated and the rest are affected slightly, showing that NAC and C-terminus are in close contact. This close contact is also shown in the profile of $\mathrm{A} 140 \mathrm{C} \alpha \mathrm{S}$; the peak intensities from position 90 to 140 are suppressed strongly revealing a compact conformation in $\mathrm{C}$-terminus region (middle and right panels in Fig. 4- 3). Considering all the profiles, it has been shown that the collapsed Cterminus of $\alpha \mathrm{S}$ is closely packed with the C-terminal end of the NAC region while Nterminus stretches out without any strong interaction.

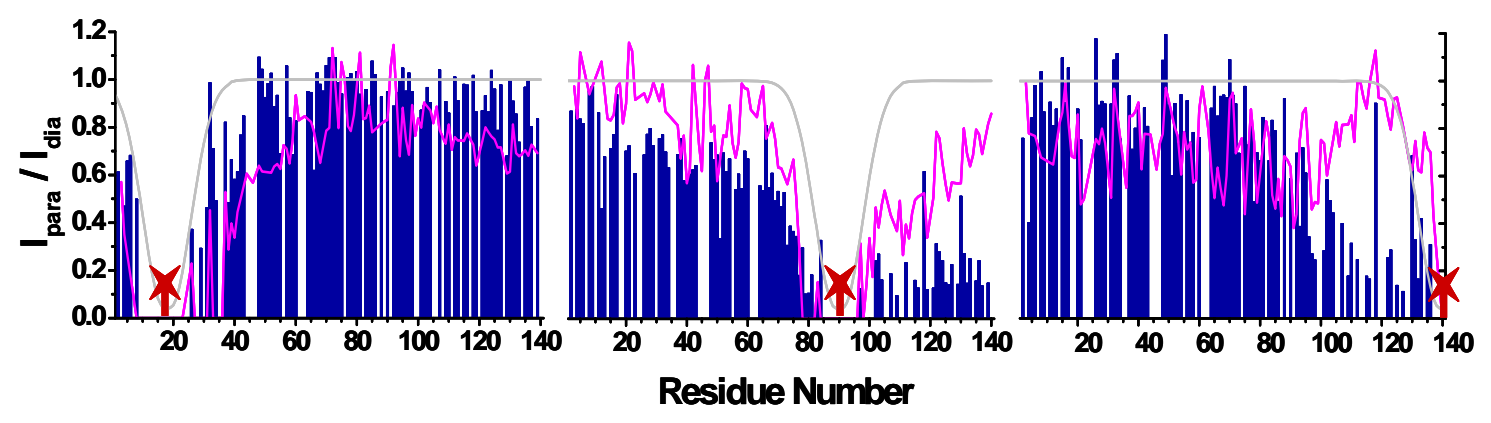

Fig. 4- 3. Comparison of PRE profiles at pH 3 (blue bars) and at pH 7.4 (magenta line).

PRE profile of $\alpha \mathrm{S}$ at $\mathrm{pH} 3$ shows release of long-range interaction between $\mathrm{N}$-terminal and C-terminal detected at pH 7.4. MTSL attached Cys are marked with red symbol. Grey line is an intensity profile in ideally extended conformation.

These conformational changes confirmed the previous observation of increased $\beta$ strand conformation in $\mathrm{CD}$ spectra and the decrease of radius of gyration $\left(\mathrm{R}_{\mathrm{g}}\right)$ in small angle X-ray scattering (SAXS) experiment (Uversky et al. 2001b).

Since the most significant change of PRE profile was observed in the C-terminus, we synthesized C-terminal peptide spanning residue 105-136 in order to have insight into 
the local conformational changes in C-terminus. The measured CD spectra of both fulllength $\alpha \mathrm{S}$ and C-terminus peptide were shown in Fig. 4- 4. Considering that the dip at $220 \mathrm{~nm}$ is the characteristic of $\beta$-strand conformation, the spectra of full-length $\alpha \mathrm{S}$ confirmed the previous observation, i.e. increased $\beta$-strand conformation at $\mathrm{pH} 3$ (dark red and dark blue) (Uversky et al. 2001b). The magnitude of the change at $220 \mathrm{~nm}$, however, is bigger for $\mathrm{C}$-terminal peptide, which shows random coil conformation at $\mathrm{pH}$ 7.4 (magenta) and adopts more $\beta$-strand conformation at $\mathrm{pH} 3$ (cyan) compared to fulllength $\alpha \mathrm{S}$ (dark blue).
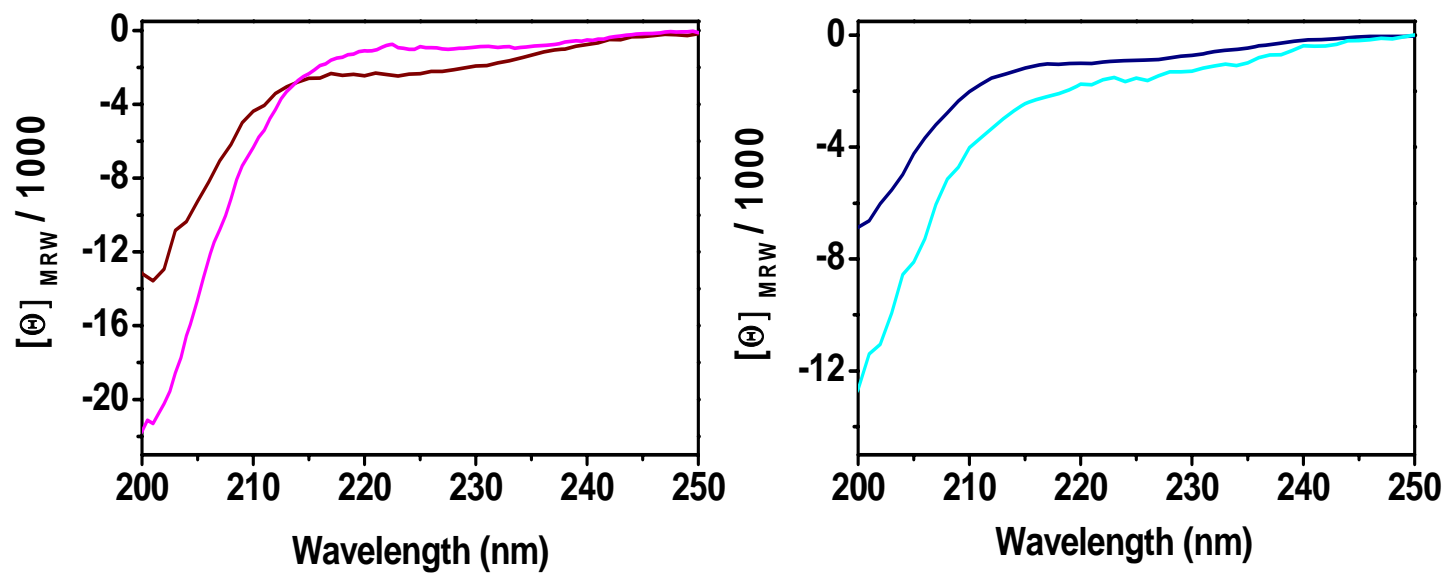

Fig. 4- 4. CD spectra of full-length wt $\alpha \mathrm{S}$ (dark) and C-terminal peptide (light).

CD spectra of both polypeptides at $\mathrm{pH} 7.4$ (left panel) and at $\mathrm{pH} 3$ (right panel). Wavelength lower than 200 is hampered by the absorbance from the buffer.

Taken together with the PRE profile and the secondary chemical shifts, this implies that $\mathrm{N}$-terminus and NAC region should be extended adopting more $\beta$-strand conformation at $\mathrm{pH} 3$, while $\mathrm{C}$-terminus is collapsed and has more $\beta$-strand conformation.

The effect of these conformational on hydrodynamic radius were investigated by 
PFG-NMR with full-length $\alpha \mathrm{S}, \alpha \mathrm{S}(1-108)$ and C-terminus peptide (Fig. 4- 5). At pH 7.4, full-length $\alpha \mathrm{S}$ and $\alpha \mathrm{S}(1-108)$ are in-between of fully unfolded and globular conformations, while C-terminus is fully unfolded. With the lowered $\mathrm{pH}, \mathrm{C}$-terminus peptide shrinks by around $4 \AA$ while $\alpha \mathrm{S}(1-108)$ extends $\sim 4 \AA$. This dimensional change agrees with PRE-derived spatial interaction; the extension of N-terminus and the collapse of C-terminus. The sum of $\mathrm{R}_{\mathrm{h}}$ changes is also in a good agreement with no net changes in $\mathrm{R}_{\mathrm{h}}$ of full-length $\alpha \mathrm{S}$ at lowered $\mathrm{pH}$.

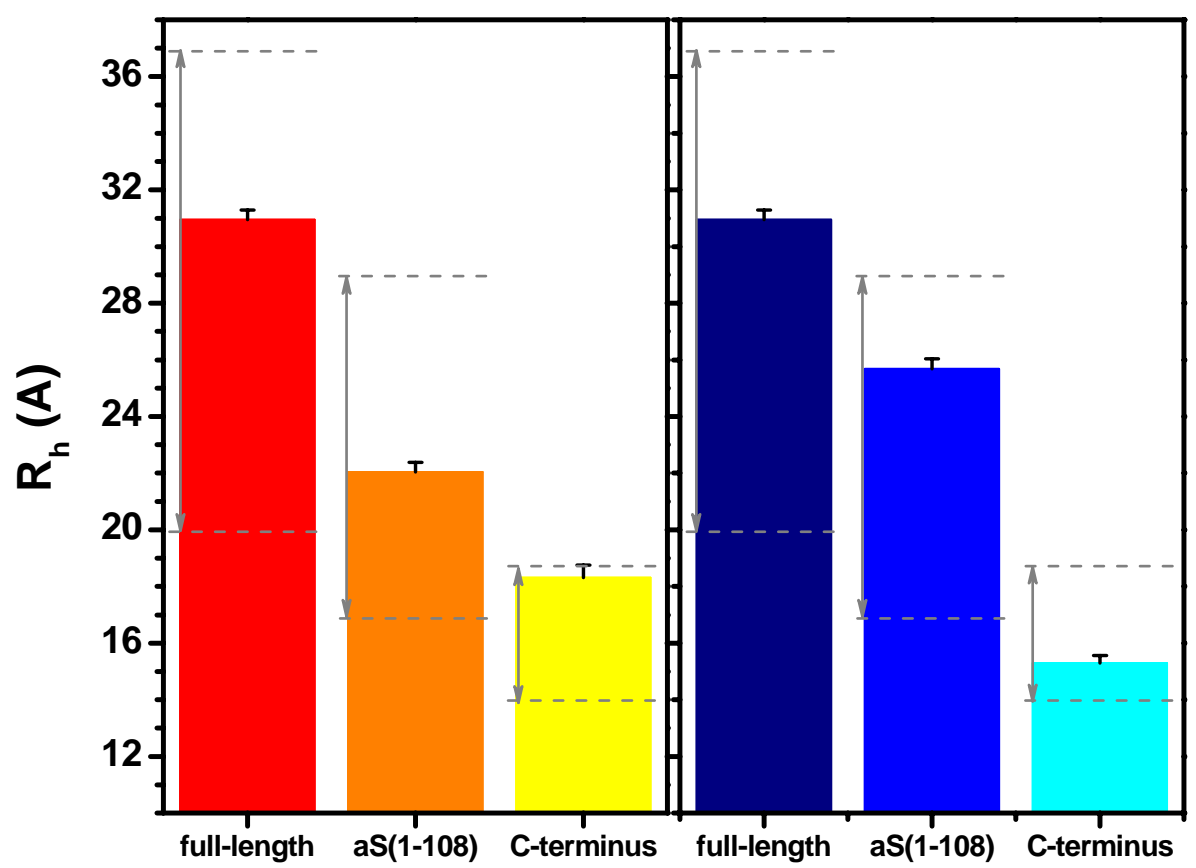

Fig. 4- 5. Hydrodynamic radii of full-length $\alpha \mathrm{S}, \alpha \mathrm{S}(1-108)$ and $\mathrm{C}$-terminus at different $\mathrm{pH}$.

The left panel shows $\mathrm{R}_{h}$ at $\mathrm{pH} 7.4$ and the right panel shows $\mathrm{R}_{h}$ at $\mathrm{pH}$ 3. The $\mathrm{R}_{h}$ limit of either globular or fully unfolded conformation of the given length of a peptide is drawn as horizontal dashed lines on each.

\subsubsection{Changes in protein dynamics and structure detected by RDCs}

In previous studies, RDCs are revealed as a useful tool to detect changes and interactions in conformational ensemble of unfolded proteins (Mohana-Borges et al. 
2004, Bernado et al. 2005a, Bertoncini et al. 2005b, Cho et al. 2007). One bond RDCs, ${ }^{1} \mathrm{D}_{\mathrm{NH}}$, of full-length $\alpha \mathrm{S}$ and C-terminal peptide (105-136) were measured at $\mathrm{pH} 3$ and analyzed along with the sequence. The profiles are compared with those at pH 7.4 (Fig. 4- 6).
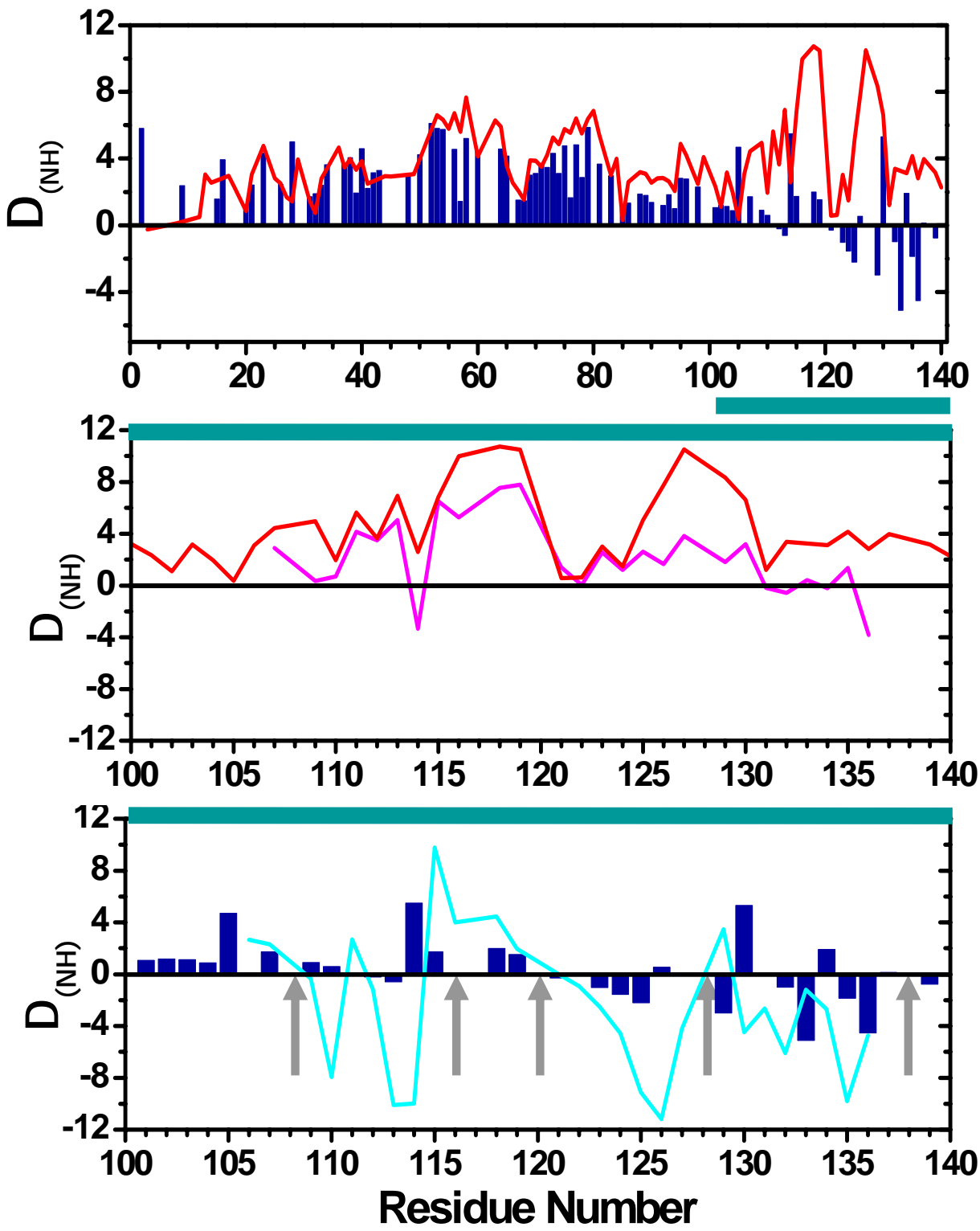

Fig. 4- 6. ${ }^{1} \mathrm{D}_{\mathrm{NH}}$ of full-length $\alpha \mathrm{S}$ and C-terminal peptide (105-136) at $\mathrm{pH} 7.4$ versus at $\mathrm{pH} 3$.

In top panel, both ${ }^{1} \mathrm{D}_{\mathrm{NH}}$ of $\alpha \mathrm{S}$ at $\mathrm{pH} 7.4$ (red line) and at $\mathrm{pH} 3$ (blue bar) are compared. C-terminus peptide (magenta) shows similar ${ }^{1} \mathrm{D}_{\mathrm{NH}}$ pattern with that of full-length $\alpha \mathrm{S}$ at $\mathrm{pH} 7.4$ in the middle panel. ${ }^{1} \mathrm{D}_{\mathrm{NH}}$ of $\mathrm{C}$ terminus peptide at pH 3 (cyan) are also compared with ${ }^{1} \mathrm{D}_{\mathrm{NH}}$ of C-terminal region of full-length $\alpha \mathrm{S}$ in bottom panel. Five Prolines are indicated by arrows. 
The sign and the amplitude of ${ }^{1} \mathrm{D}_{\mathrm{NH}}$ around residue $30 \sim 90$ shows similar pattern in both cases; $\mathrm{N}$ - and C-terminus, however, shows changes in amplitude, even alternations in sign. This ${ }^{1} \mathrm{D}_{\mathrm{NH}}$ profile implies that there should be transient local structures deviating from a random coil behavior.

Since PRE showed no long-range interaction between N- and C- terminus, the effect must be induced by local interactions. ${ }^{1} \mathrm{D}_{\mathrm{NH}}$ of C-terminal peptide (105-136) was acquired to confirm the localized effect on ${ }^{1} \mathrm{D}_{\mathrm{NH}}$ (bottom panel in Fig. 4- 6). RDCs of Cterminal peptide (105-136) at $\mathrm{pH} 7.4$ shows lower amplitudes compared with the RDCs from full-length $\alpha \mathrm{S}$, which reflects the absence of long-range interaction (middle panel in Fig. 4- 6). The same comparison at $\mathrm{pH} 3$ shows a different result. The sign-alternating pattern is reproduced in C-terminal peptide; the sign alternating points, however, are different and ${ }^{1} \mathrm{D}_{\mathrm{NH}}$ of C-terminal peptide has bigger amplitude (bottom panel in Fig. 4$6)$.

One possibility for the sign-alternating is polyproline II helix (PPII) conformation. Proline-rich peptides and poly-Ala peptides are considered to adopt polyproline II helix (PPII) conformation (Creamer and Campbell 2002, Schweitzer-Stenner and Measey 2007). At pH 3, C-terminus becomes neutral so that it has similar hydrophobicity with the poly-Pro or poly-Ala peptide. The detailed mechanism how the polyproline motif induces the sign-alternation in ${ }^{1} \mathrm{D}_{\mathrm{NH}}$ is to be investigated.

\subsection{Summary and Outlook}

This study showed that, at $\mathrm{pH} 3$, residues 1-108 of $\alpha \mathrm{S}$ adopt an extended conformation, the NAC-region preferentially populates $\beta$-structure and the C-terminal 
domain is collapsed. This conformational change should result from the changes of the negative charges in C-terminus. The several negatively charged residues in C-terminus are protonated when $\mathrm{pH}$ is low enough, and both NAC and C-terminus are hydrophobic at such condition leading hydrophobic collapse in aqueous solution.

Intriguingly, the structural changes in the C-terminal domain are hardly seen in the secondary chemical shifts, while residual dipolar couplings detects the conformational change in the C-terminal domain upon the charge removal, which may adopt polyproline helix II (PPII).

In order to interpret these NMR information in terms of $\alpha \mathrm{S}$ conformation, our experimental results will be compared with the simulated NMR parameters from an ensemble of structure using a modified flexible-meccano approach (as in Mukrasch et al. (Mukrasch et al. 2007) for the turns) combined with an accelerated MD simulation of the peptide (or part of it) at $\mathrm{pH}$ 3. The combination of NMR experiments and computer simulation will provide more detailed interpretation of experimental observation. 


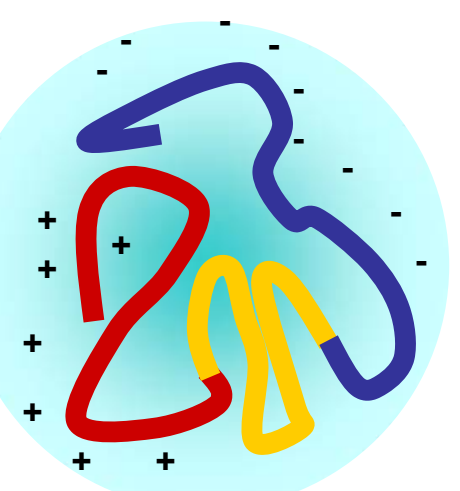

pH 7.4

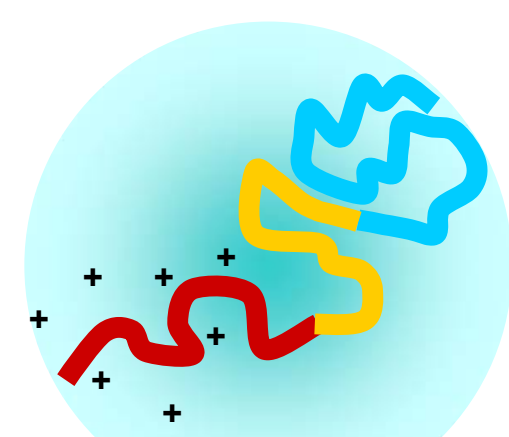

pH 3

Fig. 4- 7. Schematic model of $\alpha \mathrm{S}$ conformational change at $\mathrm{pH} 3$.

C-terminus (blue) is neutralized by low $\mathrm{pH}$ and collapses with NAC region (yellow), while positively charged N-terminus (red) is stretched out. 


\section{Cross- $\beta$ sheet core of $\alpha$-synuclein amyloid fibril determined by hydrogen/ deuterium exchange}

\subsection{Introduction}

Amongst various types of protein aggregation, amyloid fibril has attracted many researchers since it has been discovered in various neurodegenerative diseases including Alzheimer's, Parkinson's and Creutzfeldt-Jakob disease (Ross and Poirier 2004). Though diverse proteins with different morphology are able to transform into amyloid fibril, they share a common molecular feature - parts of polypeptides in amyloid fibrils adopt a cross- $\beta$ sheet quaternary structure, in vivo, or, in vitro, stabilized by highly ordered hydrogen bond network (Chiti and Dobson 2006, Eisenberg et al. 2006). In Parkinson's disease (PD), $\alpha$-synuclein $(\alpha \mathrm{S})$, intrinsically unstructured 140-residue protein consisted of charged $\mathrm{N}$ - and $\mathrm{C}$ - terminus and hydrophobic non amyloid- $\beta$ component (NAC) region, has been found as an major component of intracytoplasmic neuronal inclusions (Lewy bodes) (Uversky 2007). Genetic studies found three missense mutants (A30P, A53T and E46K) are linked to autosomal dominant form of early-onset PD (Polymeropoulos et al. 1997, Kruger et al. 1998, Juan J. Zarranz 2004). In order to understand the differences between wild type (wt) and those mutants which may provide a critical insight into the $\alpha \mathrm{S}$-induced neurodegeneration in $\mathrm{PD}$, intensive biochemical and biophysical studies with various methods including NMR (Bussell and Eliezer 2001, Bertoncini et al. 2005b), atomic force microscopy (AFM) (Segers-Nolten et al. 2007), fluorescence (Ohnishi et al. 2004), FTIR (Petkova et al. 2002), electron 
microscopy (EM) (Lashuel et al. 2002), have been performed on several conformational states of synuclein. As one of such efforts, the fibrillar core of $w t \alpha \mathrm{S}$ was determined at high resolution by solid state NMR (ssNMR) spectroscopy (Heise et al. 2005, Vilar et al. 2008), mass spectroscopy (MS) (Del Mar et al. 2005), and electron paramagnetic resonance (EPR) spectroscopy (Chen et al. 2007). The experimental results showed the boundary of rigid cross- $\beta$ structure in $\alpha \mathrm{S}$ fibril with some deviations in $\beta$-strand and loop positions.

As a tool to investigate amyloid fibril structure, various NMR experimental techniques have been used. High-resolution magic angle spinning (HR-MAS) NMR experiment is one of recent NMR technique that has been used widely in investigation of heterogeneous systems (Griffin 2003). HR-MAS was applied to tau aggregates (Sillen et al. 2005) and HET-s fibril (Siemer et al. 2006), and revealed the existence of highly flexible loops inside amyloid fibrils. Hydrogen/deuterium (H/D) exchange combined with solution state NMR (Krishna et al. 2004) or MS (Englander 2006) has been used widely to determine residue-specific folding mechanism, stability and dynamics in proteins. Its application extended, with rapid dissolving method of fibril into detectable monomer, to amyloid fibrils due to the common cross- $\beta$ motif in fibril core (Hoshino et al. 2002). Due to declination of H/D exchange rate by hydrogen bonds, in deuterated buffer, residues involved in hydrogen bonds sustain protons longer than those of flexible region. Many amyloid fibril cores were identified by H/D exchange with MS (Kheterpal et al. 2000, Del Mar et al. 2005, Kheterpal and Wetzel 2006) or with solution state NMR (Hoshino et al. 2002, Carulla et al. 2005, Luhrs et al. 2005, Hoshino et al. 2007).

Here, we investigated wt fibril with HR-MAS NMR experiment and H/D exchange 
combined with NMR spectroscopy. The combination of HR-MAS and H/D exchange allows identification of flexible regions, statistically disordered regions, and hydrogenbonded stretches in wt $\alpha \mathrm{S}$ fibril. The method was extended to explore differences in the fibrillar core of $w t$ and $\mathrm{A} 30 \mathrm{P} \alpha \mathrm{S}$. The identified fibrillar core structure of $w t \alpha \mathrm{S}$ was comparable to the known fibrillar core (Heise et al. 2005), and A30P $\alpha \mathrm{S}$ showed similar fibril core boundary to wt $\alpha \mathrm{S}$ with some differences in distribution or stability of $\beta$ strands.

\subsection{Materials and methods}

\subsubsection{Preparation of $\alpha \mathrm{S}$ amyloid fibril}

${ }^{15} \mathrm{~N}$-labeled wt and mutant $\alpha \mathrm{S}$ were expressed and purified as described in Chapter 2. Each purified $100 \mu \mathrm{M}$ monomer solution (buffered $20 \mathrm{mM}$ Tris, $100 \mathrm{mM} \mathrm{NaCl}$ at $\mathrm{pH}$ 7.4) was aliquot by $500 \mu \mathrm{L}$ into glass vials and incubated at $37^{\circ} \mathrm{C}$ with stirring for 14 days. Fibril maturation and overall morphology were confirmed by Thioflavin T (ThT) fluorescence assay and by EM, respectively, as described (Hoyer et al. 2002). Fibrils in the solution were collected by ultracentrifugation with $60,000 \mathrm{rpm}$ for 2 hours at $4{ }^{\circ} \mathrm{C}$. The supernatant was discarded and the pellet was re-dissolved into distilled $\mathrm{H}_{2} \mathrm{O}$ then ultracentrifuged. This step was repeated twice to remove salts and remaining monomers.

\subsubsection{HR-MAS NMR experiment}

Prepared fibril was resuspended in $50 \mathrm{mM}$ HEPES at $\mathrm{pH} 7.4,100 \mathrm{mM} \mathrm{NaCl}, 10 \%$ $\mathrm{D}_{2} \mathrm{O}$ aqueous solution. 1D ${ }^{1} \mathrm{H}$ spectra were measured on Bruker AVANCE NMR 900 
$\mathrm{MHz}$ equipped with $5 \mathrm{~mm}$ triple-resonance, pulsed-field z-gradient cryoprobe, and showed no protein signal due to high molecular weight of fibril. Then the fibril sample was transferred into $4 \mathrm{~mm}$ HR-MAS sample rotor. The rotor was placed into Bruker AVANCE NMR $900 \mathrm{MHz}$ equipped with HR-MAS probehead and $2 \mathrm{D}{ }^{1} \mathrm{H}_{-}{ }^{15} \mathrm{H}$ HSQC spectrum with WATERGATE scheme (Piotto et al. 1992, Mori et al. 1995, Liu et al. 1998) was measured with the sample rotation at $7.2 \mathrm{kHz}$. Acquired spectrum was analyzed with Sparky 3.111 (Goddard and Kneller)

\subsubsection{Hydrogen-deuterium exchange}

Collected fibril pellet was resuspended into $0.1 \%$ formic acid (pD 4.0), $200 \mathrm{mM}$ $\mathrm{NaCl}$ in $99.9 \% \mathrm{D}_{2} \mathrm{O}$ and placed on a rotator at $4{ }^{\circ} \mathrm{C}$ for $\mathrm{H} / \mathrm{D}$ exchange with reduced monomer-fibril exchange process (Del Mar et al. 2005). After specified period, fibril was collected by ultracentrifugation, frozen by liquid nitrogen and stored in a refrigerator at $-80{ }^{\circ} \mathrm{C}$. Lyophilized monomer and fibril without $\mathrm{H} / \mathrm{D}$ exchange was also stored in the same way till the NMR experiment.

\subsubsection{NMR experiment with rapid fibril dissolving}

$2 \mathrm{M} \mathrm{GuSCN}, 0.4 \%$ formic acid (pD 2.4) in $50 \% \mathrm{D}_{2} \mathrm{O}$ aqueous solution was prepared as a dissolving buffer. All the samples and the solution were kept in an icebox. Each sample was dissolved into the dissolving buffer and placed into Bruker AVANCE 600 MHz NMR equipped with $5 \mathrm{~mm}$ triple-resonance, pulsed-field z-gradient cryoprobe, which was adjusted and calibrated at $5{ }^{\circ} \mathrm{C}$ in advance. Including shimming, the preparative step took around 8 minutes before the first experiment started. Series of ${ }^{1} \mathrm{H}-$

${ }^{15} \mathrm{~N}$ HSQC spectra were measured for 14 hours. Acquired spectra were processed with 
linear prediction on ${ }^{15} \mathrm{~N}$ dimension and analyzed by NMRPipe (Delaglio et al. 1995).

Resonance assignment was done with HNN experiment (Panchal et al. 2001) and Sparky 3.111 (Goddard and Kneller).

\subsection{Results and discussion}

\subsubsection{Flexible regions in $\alpha S$ amyloid fibril detected by HR-MAS}

After ThT fluorescence reached the saturation point, EM picture was take by Dr. Dietmar Riedel. EM shows typical amyloid fibril structures of $\alpha \mathrm{S}$ (Fig. 5- 1). The sample was washed and prepared for NMR experiment. The empty $1 \mathrm{D}{ }^{1} \mathrm{H}$ spectra of the fibril in conventional solution-state NMR proved that no monomeric $\alpha \mathrm{S}$ was in the sample.
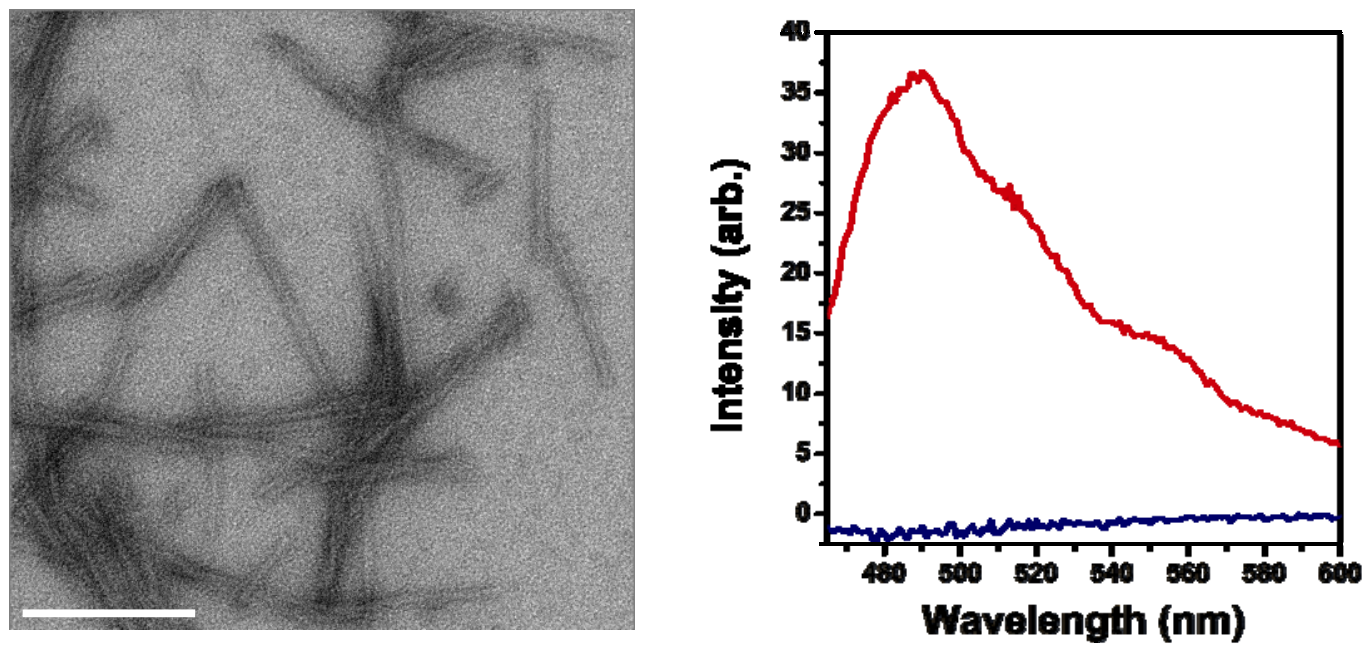

Fig. 5- 1. EM image and ThT florescence of $\alpha S$ amyloid fibril.

Electron microscopy image on the left panel shows typical amyloid fibril structures of $\alpha \mathrm{S}$ (white bar is $200 \mathrm{~nm}$ scale). ThT emits fluorescence upon binding to cross- $\beta$ sheet in $\alpha \mathrm{S}$ amyloid fibril (red) while emits no fluorescence with monomeric $\alpha \mathrm{S}$ (blue). 
The prepared fibril sample were then transferred into $4 \mathrm{~mm}$ HR-MAS sample rotor and $1 \mathrm{D}{ }^{1} \mathrm{H}$ spectrum was acquired in HR-MAS probe with spin-rate of $7.2 \mathrm{kHz}$, from which proton NMR signals were detected. We extended the NMR experiment to $2 \mathrm{D}{ }^{1} \mathrm{H}$ ${ }^{15} \mathrm{~N}$ HSQC spectra and detected cross-peak from the backbone amide nuclei (Fig. 5- 2). Since HR-MAS NMR detects mobile nuclei, this reveals that the $\alpha \mathrm{S}$ amyloid fibril has some highly flexible regions even though the monomers in amyloid fibril are linked by tightly organized cross- $\beta$ structure. The overall cross-peak pattern in the spectrum appears similar with that of $\alpha \mathrm{S}$ monomer and it enabled us to assign resonances by comparison. The peak picking and the assignment was done with Sparky 3.111 with ${ }^{15} \mathrm{~N}$ cutoff 0.2 ppm and ${ }^{1} \mathrm{H}$ cutoff $0.02 \mathrm{ppm}$. 


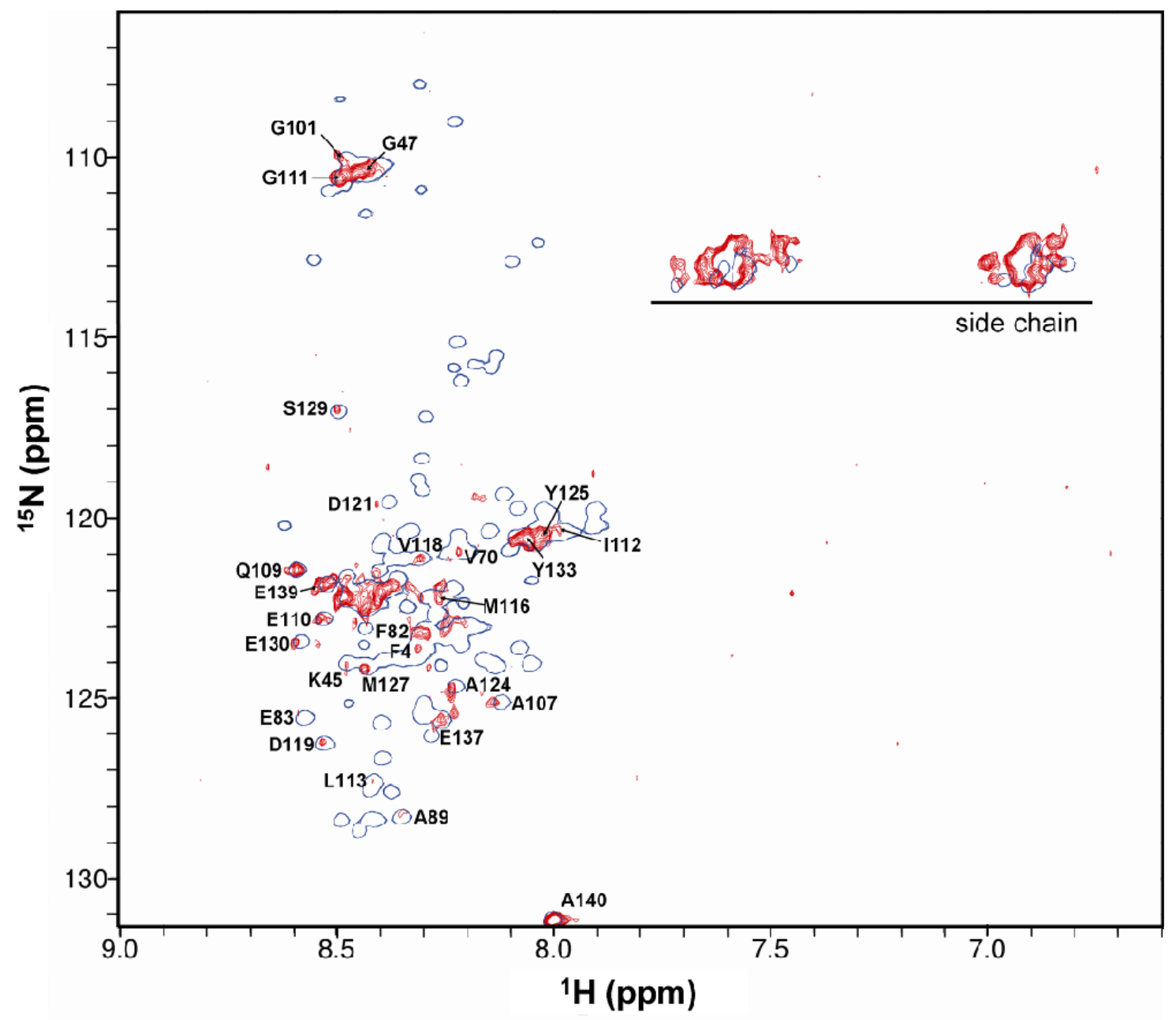

Fig. 5- 2. Comparison of $2 \mathrm{D}{ }^{1} \mathrm{H}-{ }^{15} \mathrm{~N} H S Q C$ spectra from $w t a S$ fibril (red) and from monomer (blue).

Peak assignment was done by comparison with monomeric assignment with ${ }^{15} \mathrm{~N}$ cutoff 0.2 ppm and ${ }^{1} \mathrm{H}$ curoff $0.02 \mathrm{ppm}$. 


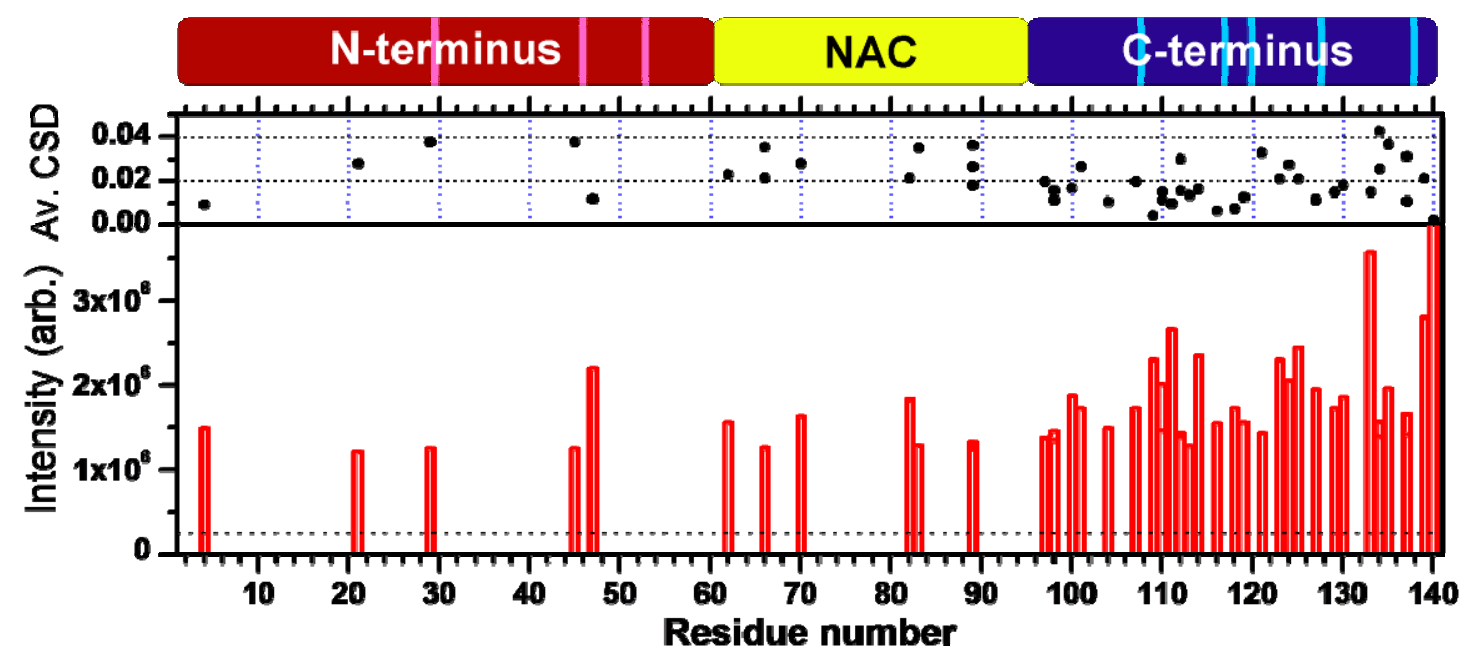

Fig. 5- 3. Residue specific peak intensity from $2 D{ }^{1} \mathrm{H}-{ }^{15} \mathrm{~N} H S Q C$ spectra of $w t \alpha S$ fibril in HR-MAS. The top panel shows schematic description of $\alpha \mathrm{S}$. Three genetic mutation sites are colored with magenta; Prolines are colored with cyan. Average chemical shift difference of ${ }^{1} \mathrm{H}$ and ${ }^{15} \mathrm{~N}$ is calculated with (Eq. 21) and depicted in the middle panel.

The intensities of the assigned peaks in $2 \mathrm{D}{ }^{1} \mathrm{H}_{-}{ }^{15} \mathrm{~N}$ HSQC spectrum of wt $\alpha \mathrm{S}$ amyloid fibril in HR-MAS probe are summarized in Fig. 5- 3. The averagedchemical shifts deviation (CSD) from ${ }^{1} \mathrm{H},{ }^{15} \mathrm{~N}$ chemical shifts of the cross peaks between $\alpha \mathrm{S}$ monomer and $\alpha \mathrm{S}$ amyloid fibril has rather small values $(<0.05 \mathrm{ppm})$. Most of the cross peaks are assigned from C-terminus, and that confirms the previously observed flexible Cterminus even in the fibril state (Del Mar et al. 2005, Heise et al. 2005, Vilar et al. 2008). Intriguingly, some peaks are assigned from NAC and N-terminus. Theses crosspeaks may be from the specified residues that are in a flexible loop region, or may be originated from the wrong assignment.

\subsubsection{Hydrogen/Deuterium exchange and rapid dissolving}

H/D exchange combined with spectroscopy has been widely used for the protein folding study and for the fibril structure determination (Hoshino et al. 2002, Englander 
2006, Hoshino et al. 2007, Baldwin 2008). The remarkable size of amyloid fibrils hampers direct detection of H/D exchange from the fibril by solution state NMR; hence, a critical step is the rapid dissociation of fibril into the detectable size with preserving $\mathrm{H} / \mathrm{D}$ exchange information. Deuterated dimethyl sulfoxide (DMSO- $d_{6}$ ) with $5 \% \mathrm{H}_{2} \mathrm{O}$ (Hoshino et al. 2007) and $6 \mathrm{M} \mathrm{GuSCN,} \mathrm{0.4 \%} \mathrm{formic} \mathrm{acid} \mathrm{(pD} \mathrm{2.4)} \mathrm{in} 99.9 \% \mathrm{D}_{2} \mathrm{O}(\mathrm{Del}$ Mar et al. 2005) are the solvents used for that purpose. With DMSO- $d_{6}$, typically a series of fractions from the fibril solution are aliquoted at a certain exchange time, lyophilized and the fibril are dissociated into DMSO- $d_{6}$ solution. With NMR spectroscopy, a series of ${ }^{1} \mathrm{H}_{-}{ }^{15} \mathrm{~N}$ spectra are measured to follow the decay of the hydrogen signal in DMSO- $d_{6}$ solution and one of HSQCs of each aliquot at the same time point is extracted. The peak intensities of each extracted HSQC are compared with those of the reference (no H/D exchange) and are fitted with single exponential curve as a function of $\mathrm{H} / \mathrm{D}$ exchange time. Some of these experiments require the experiment time in scale of several months due to the highly stable cross- $\beta$ structure of amyloid fibril.

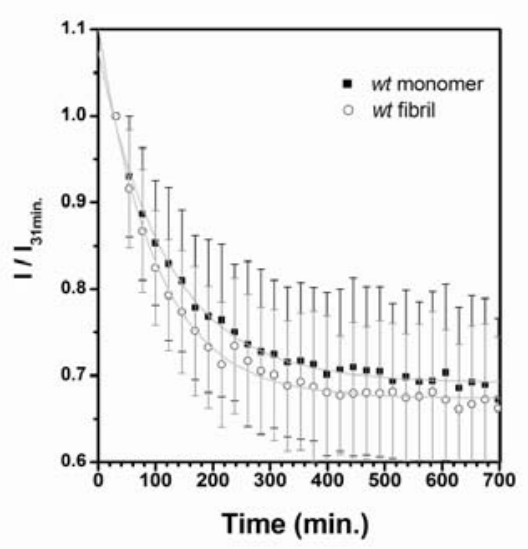

Fig. 5- 4. Average peak intensity decay curves in $50 \%$ D2O dissolving buffer.

The peak intensity changes of both wt $\alpha \mathrm{S}$ monomer and amyloid fibril show comparable decay curves, 
which confirms that amyloid fibril dissolves in rapid time scale.

We modified this method to reduce the amount of materials and to distinguish exposed and buried residues easier. The main modification is the use of $50 \% \mathrm{D}_{2} \mathrm{O}$ solution with $2 \mathrm{M} \mathrm{GuSCN}, 0.4 \%$ formic acid, pD 2.4. Since $50 \%$ of $\mathrm{D}_{2} \mathrm{O} / \mathrm{H}_{2} \mathrm{O}$ mixture is used, the peak intensity from the HSQC after equilibrium should be from $50 \%$ of the total amount of the monomers in the solution, which enables us to obtain the initial peak intensity (at time 0 in the dissolving buffer) by extrapolation of a simple exponential decay curve with 0.5 offset. Once H/D exchanged fibrils are used, the initial peak intensity should represent the decayed amount of hydrogen by H/D exchange in the fibril. The use of internal reference intensity can reduce the possible experimental error that may be introduced by unequal amount amyloid fibrils in each aliquot. Moreover, the residues with more than $50 \%$ exchanged show exponential growth curve, which enables us to distinguish between buried and exposed residues easier. Our condition was tested with fully-protonated wt $\alpha \mathrm{S}$ monomer and wt $\alpha \mathrm{S}$ fibril in order to ensure the rapid dissociation of amyloid fibril while preserving the H/D exchange information. Fig. 5- 4 shows the comparison of $\alpha \mathrm{S}$ monomer and $\alpha \mathrm{S}$ fibril intensities in the dissolving solution. The average decay curves follow a single exponential decay curve and the exchange times are comparable. There is H/D exchange during the first HSQC spectrum (acquired at $31 \mathrm{~min}$. after mixing the solution and the protein), hence the final intensity ratio reaches around 0.7 compared to the first acquired intensity.

\subsubsection{Intensity profiles of 1 week $H / D$ exchanged fibrils}

wt and A30P mutant $\alpha \mathrm{S}$ amyloid fibrils were prepared and resuspended in $99.9 \%$ 
$\mathrm{D}_{2} \mathrm{O}$ with $0.1 \%$ formic acid, $\mathrm{pD} 4$. After one week of $\mathrm{H} / \mathrm{D}$ exchange at $4{ }^{\circ} \mathrm{C}$, the fibrils were collected by ultracentrifugation and resuspended into the dissolving buffer. The peak intensity modulations along with a series of ${ }^{1} \mathrm{H}_{-}{ }^{15} \mathrm{~N}$ HSQC spectra were analyzed with NMRDraw and the change of each intensity ratio from selected residues is shown in Fig. 5- 5. All the peak ratios were calculated based on the intensity from the first ${ }^{1} \mathrm{H}-$ ${ }^{15} \mathrm{~N}$ HSQC $\left(\mathrm{I}_{31 \mathrm{mn} .}\right)$ spectrum.

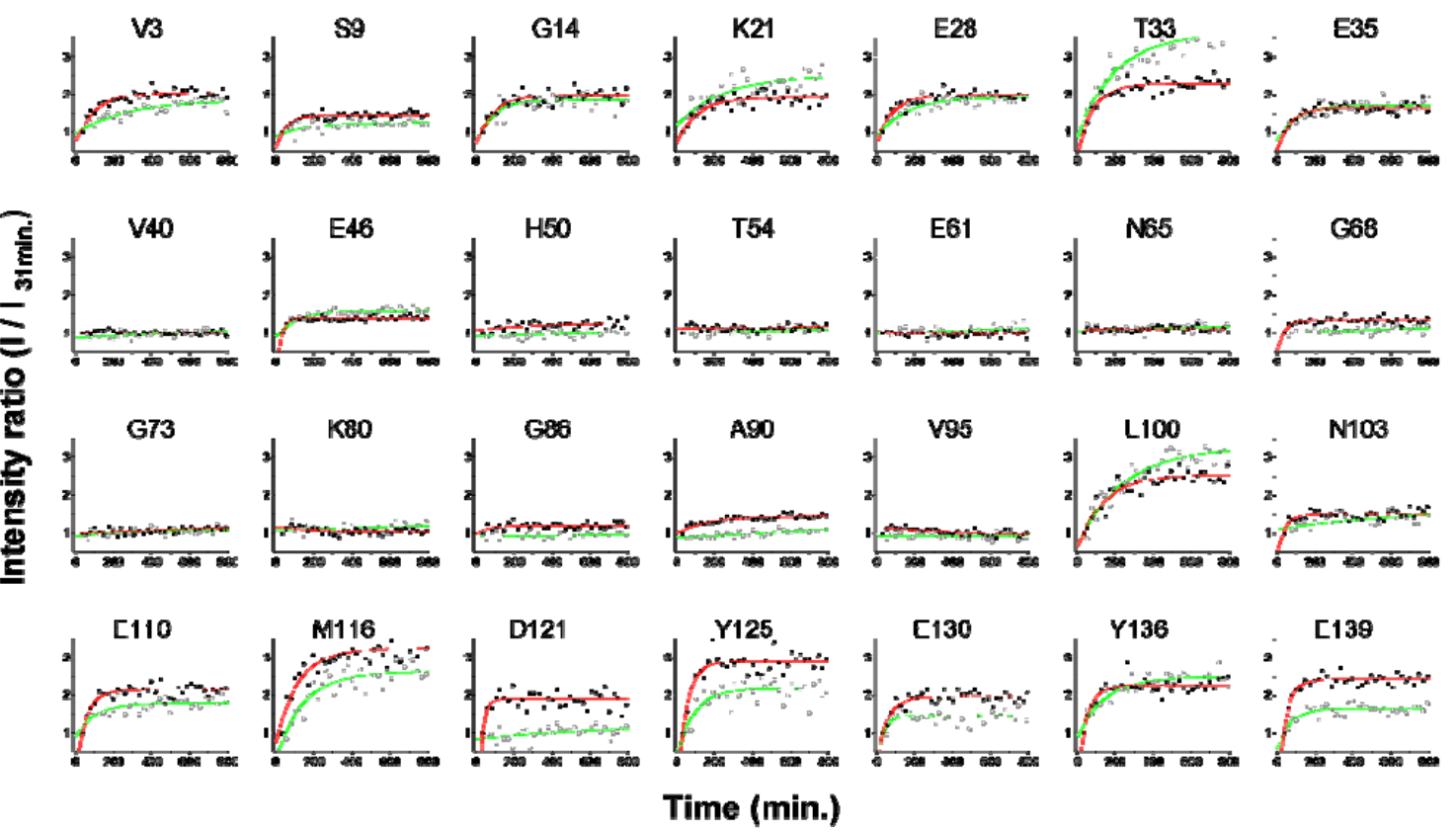

Fig. 5- 5. Peak intensity modulations of one week exchanged fibrils.

The intensity profiles as a function of dissolved time are depicted with filled circles for $w t$ fibril and open circles for A30P fibril. Each profile is fitted with a single exponential curve (red, wt $\alpha \mathrm{S}$; green, $\mathrm{A} 30 \mathrm{P} \alpha \mathrm{S}$ ).

From V3 to E35 in N-terminus and from L100 to E139 in C-terminus, the intensity ratio increases by many folds, implying that those residues obtain more protons during the dissolving process and, in accordance, amide protons in those residues should be accessible to the solvent during H/D exchange period. Conversely, the residues from 
V40 to V95 show smaller increases or even decreases, which implies that those residues were protected from H/D exchange. Through the extrapolation of single exponential fitting curve, the initial intensity ratio was obtained.

For some residues in Fig. 5- 5, there are differences in the saturation heights of the growth curves for the identical residues. Such results can be due to the variation of H/D exchange time between $w t$ and $\mathrm{A} 30 \mathrm{P} \alpha \mathrm{S}$ amyloid fibril since the potential differences in local conformation during dissolving procedure can be excluded by the comparable exchange rate constants for the identical residues between wt and $\mathrm{A} 30 \mathrm{P} \alpha \mathrm{S}$ amyloid fibril. In contrast, each rate differs from each other when it is compared in one protein, which can be explained neighboring residue effect on hydrogen/deuterium exchange rate ((Bai et al. 1993, Croke et al. 2008).

\subsubsection{Fibril core region specified by $H / D$ exchange}

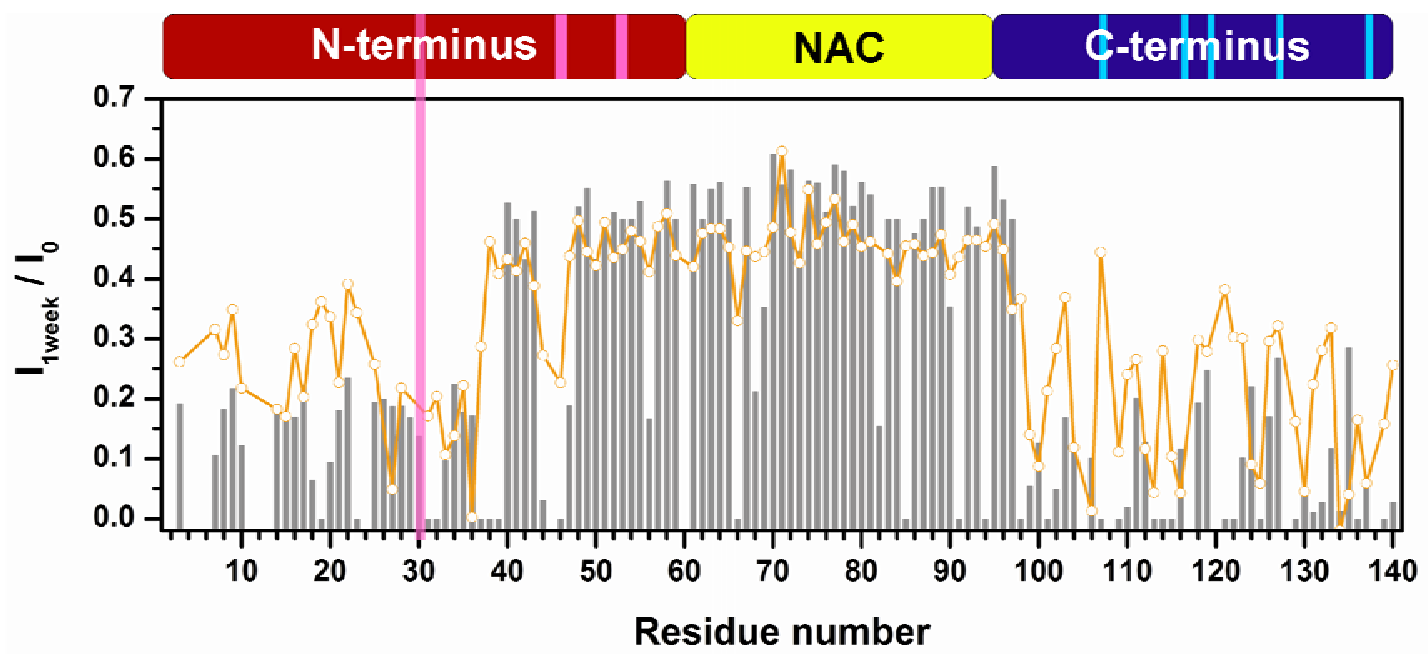

Fig. 5- 6. Back calculated peak intensity after 1 week $H / D$ exchange.

Both wt (grey bar) and A30P (open circle with orange line) show similar boundaries of fibril core region. 
A30P have higher values in $\mathrm{N}$-terminus and a part of $\mathrm{C}$-terminus.

The initial intensity ratio calculated should be equal to the ratio of remaininig hydrogens in the peptide bonds of the amyloid fibril after one week of H/D exchange. The initial intensity ratio after one week of $\mathrm{H} / \mathrm{D}$ exchange $\left(\mathrm{I}_{1 \text { week }} / \mathrm{I}_{0}\right)$ from both wt and A30P mutant $\alpha \mathrm{S}$ amyloid fibrils are plotted as a function of residue number in Fig. 5- 6. The boundaries of fibril core region, comprising residues with higher ratio, could be identified. Notably, A30P $\alpha \mathrm{S}$ amyloid fibril showed more extended core region (position 37- 98) than wt $\alpha \mathrm{S}$ amyloid fibril (residue 40-97). A recent ssNMR investigation on the amyloid fibril of A53T $\alpha \mathrm{S}$ mutant showed an extended boundary of fibril core region (residue number 38 100) compared to wt $\alpha \mathrm{S}$ amyloid fibril (position 38 95) (Heise et al. 2008). The differences in the length of fibril core region may due to the genetic mutants; however, it should be noted that the length of the fibril core even from the same wt $\alpha \mathrm{S}$ fibril varies in several investigations (Del Mar et al. 2005, Heise et al. 2005, Chen et al. 2007, Vilar et al. 2008). A potential cause of this deviation is slow dynamics in amyloid fibrils, which can rearrange the secondary structural elements in the fibrils (Heise et al. 2005).

Another characteristic of A30P amyloid fibril can be observed from the residues outside of the fibril core region, which have higher intensity ratios compared to wt $\alpha \mathrm{S}$ amyloid fibril. That can be due to the difference in H/D exchange time for wt and A30P $\alpha \mathrm{S}$ amyloid fibril, but the structural motif or the dynamics in those regions also cane be the reason, which is to be elucidated.

\subsection{5. $\beta$-sheet structure arrangement in the fibril core}


The identified fibril core regions were investigated in detail. As the fibril core region mainly consists of cross- $\beta$ structure, the residues with higher intensity ratio $\left(\mathrm{I}_{1 \text { week }} / \mathrm{I}_{0}\right)$ should be involved in $\beta$-sheet conformation in the fibril state. The intensity profile in the region from residue number 30 to 100 is compared with the previously determined secondary structures in Fig. 5- 7.

Note that the loop regions with lower intensity ratios are comparable with the HRMAS derived flexible regions. Considering that the assignment is solely based on the comparison between 2D ${ }^{1} \mathrm{H}^{15}{ }^{15}$ HSQC of amyloid fibril and that of monomer, this consistency enables us to infer that the flexible $\mathrm{N}$-terminal and C-terminal domains of NAC region, even in the fibrillar state, may experience almost same chemical environment as the loop exposed in the solution.

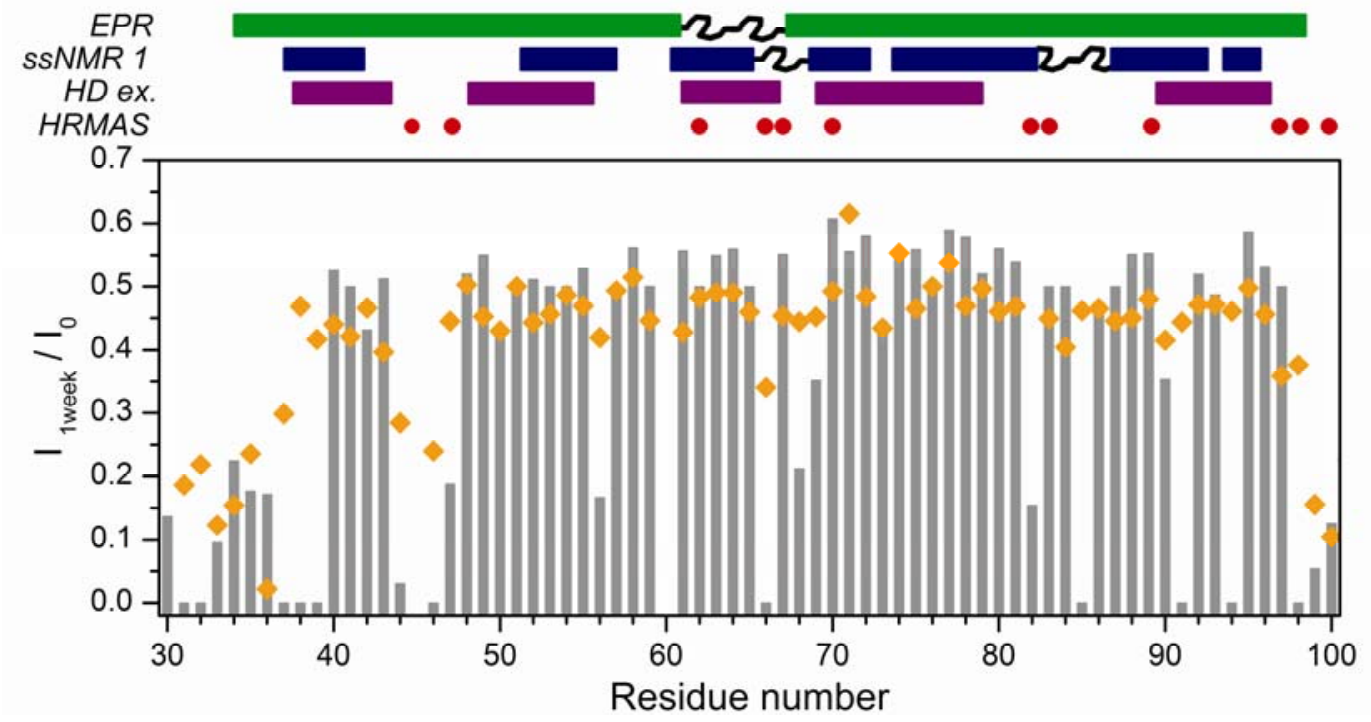

Fig. 5- 7. Peak intensity profile in the fibril core region of $\alpha \mathrm{S}$.

The peak intensity of residue 30 to 100 are shown for both wt (grey bar) and A30P (orange circle). The secondary structural elements determined from the literatures are described as a bar for $\beta$-strand and as a line for link.(green, EPR study(Chen et al. 2007) ; blue, ssNMR study (Heise et al. 2005); purple, H/D exchange and NMR study (Vilar et al. 2008)). Red circles represent the putative flexible regions from 
HR-MAS.

$\beta$-strands in the fibril core region were identified with the residues that have higher initial intensity ratio $\left(\mathrm{I}_{1 \text { week }} / \mathrm{I}_{0}\right)$. The determined $\beta$-strands in $w t \alpha \mathrm{S}$ amyloid fibrils are as following: $\beta \mathrm{I}$ (40-43), $\beta \mathrm{II}$ (48-55), $\beta \mathrm{III}$ (57-65), $\beta \mathrm{IV}$ (70-81), $\beta \mathrm{V}$ (86-90), and $\beta \mathrm{V}^{\prime}$ (9597). The $\beta$-strands in A30P $\alpha \mathrm{S}$ amyloid fibril are identified as: $\beta \mathrm{I}(38-43), \beta \mathrm{II}(47-55)$, $\beta \mathrm{III}$ (57-65), $\beta \mathrm{IV}$ (67-81), $\beta \mathrm{V}$ (85-89), and $\beta \mathrm{V}^{\prime}$ (92-96). Both amyloid fibril cores contain similar arrangement of $\beta$-strands and loops. Note that this way of identification has some drawback: (1) the edge of each $\beta$-strand has more flexibility so that the residue is less protected, which can make the exact length of $\beta$-strand vary with individual criteria, (2) the outmost $\beta$-strand from amyloid fibril is exposed to solution and may have different $\mathrm{H} / \mathrm{D}$ exchange rate.

By the comparison with the previous investigations, identified are some tricky regions comprising 57-59, 83-84 and 92-93. These residues may adopt $\beta$-strand or other conformation, which is due to the possible dynamic feature of amyloid fibril shown by Heise et al. (Heise et al. 2005). As mentioned above, the dynamics in amyloid fibril can differ in wt and A30P mutant amyloid fibrils and that may induce both the different length of $\beta$-strands and the smaller variance in intensity ratio between $\beta$-strand and loop in A30P $\alpha \mathrm{S}$ fibril. This hour to day time scale dynamics needs further confirmation.

\subsection{Summary and Conclusion}

This work showed that highly flexible residues in wt $\alpha \mathrm{S}$ amyloid fibril can be 
identified with HR-MAS and the peak assignment reveals that some of the residues are from fibril core region (residue number 35-100). The arrangement of $\beta$-strand and flexible loop in the fibril core region were identified with $\mathrm{H} / \mathrm{D}$ exchange experiment followed by rapid dissolving in $50 \% \mathrm{D}_{2} \mathrm{O} / \mathrm{H}_{2} \mathrm{O}$ solution with a series of $2 \mathrm{D}{ }^{1} \mathrm{H}_{-}{ }^{15} \mathrm{~N}$ HSQCs, which gave a better distinction between the exchanged residues and the protected residues. Detailed analysis showed that the arrangement and the number of $\beta$ strand are comparable, but the length of each $\beta$-strand is slightly different. The same information was acquired for A30P mutant amyloid fibril and revealed that A30P has more extended boundaries of amyloid fibril core in $\beta I$ (38-43 vs. 40-43). However, previous investigations showed that the fibril core of $w t \alpha \mathrm{S}$ can also reach to position 38 , and it gave us an indication of potential dynamics in amyloid fibril. The dynamics of amyloid fibril should be in hour to day time scale and needs to be investigated with appropriate methods. ssNMR can be a good option since it doesn't disrupt the amyloid fibril and detect dynamics in atomic resolution. 


\section{Conformation study on $\alpha$-synuclein oligomers derived from amyloid fibrils in supercooled solution}

\subsection{Introduction}

Recently, we revealed that $\alpha \mathrm{S}$ amyloid fibrils dissociate into monomers in supercooled water (Kim et al. 2008). The dissociation was confirmed by EM, NMR, and ThT fluorescence. Intriguingly, EM of the dissociates shows dark and crowded background that was not observed in EM of fibril (Kim et al. 2008). The amount of the dissociated monomer, determined as $\sim 15 \%$ with NMR spectra, and remaining amyloid fibril, $\sim 30 \%$ of initial fibril by ThT fluorescence emission, indicated that there must be another species which cannot be detected by NMR and ThT considering.

This species with high molecular weight should be oligomers, and further characterization has been performed with microscopic methods. Below is a brief description about EM and AFM study on the oligomer. All EM images were taken by Dr. Dietmar Riedel.

\subsection{Morphology of the oligomers}

To define the dimensional characteristic of the oligomers, microscopic images were acquired. Prior to the characterization, the oligomers had to be separated from the fibril that hampers clear view of smaller species. Centrifugation for $15 \mathrm{~min}$. with 13,200 $\mathrm{g}$ and careful aliquot of upper $50 \%$ supernatant was sufficient to make protofibril-free 
solution sample.

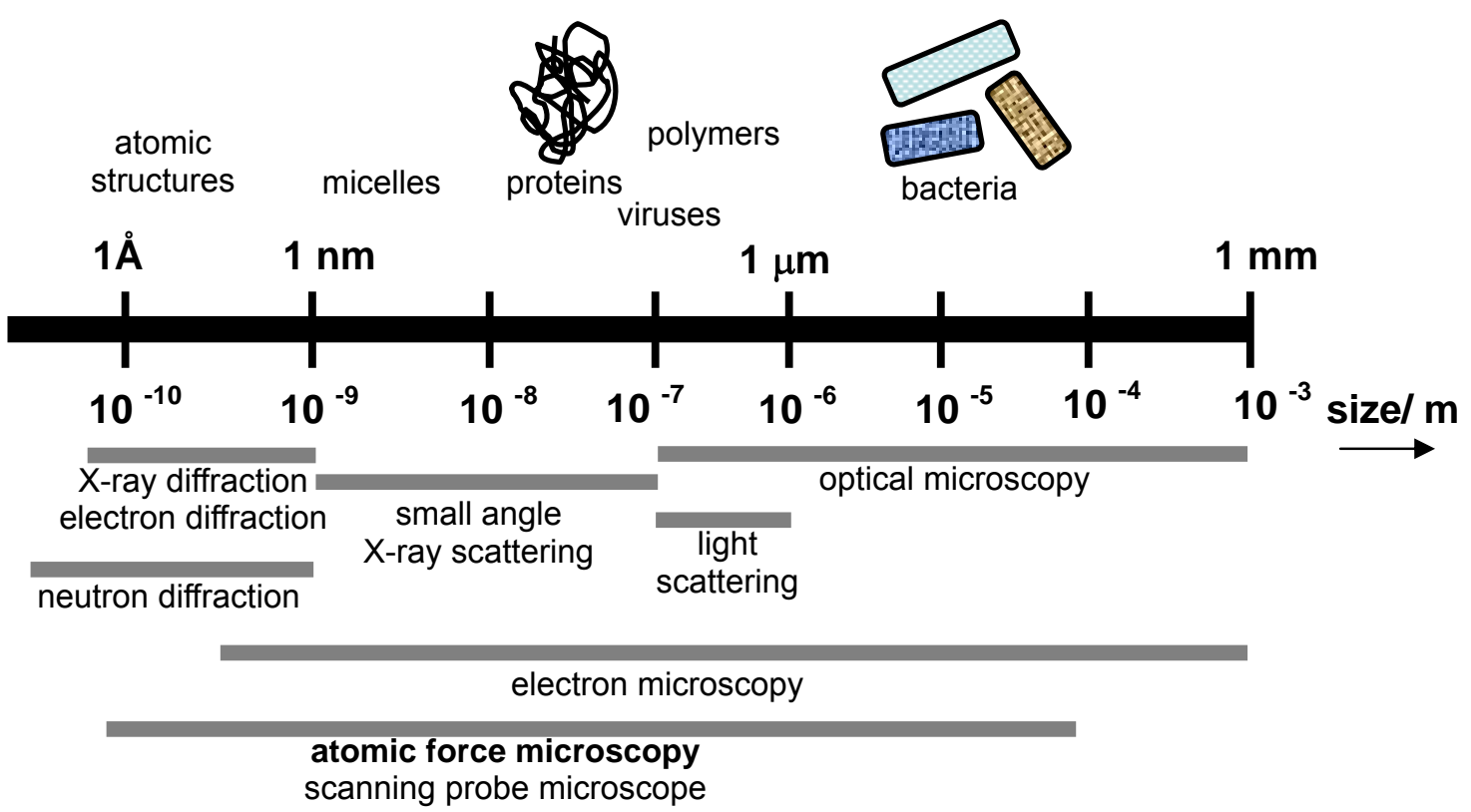

Fig. 6- 1. The resolution of microscopic methods and the dimension of biomolecules and chemicals. Adapted from Jansen (Jansen 2005) and modified by Hai-Young Kim

TEM images and ThT fluorescence emission of fibril, fibril dissociates, and the oligomers are shown in Fig. 6- 2. The fibril has typical morphology of amyloid fibril (left in Fig. 6- 2a) while EM of the fibril dissociates shows mixture of protofibril and spherical oligomers (middle in Fig. 6- 2a). After the purification, clearer image of spherical species, the oligomer, were acquired (right in Fig. 6- 2a). The spherical species are categorized into two types based on the diameter, 15-20 nm and 20-30 nm, which were observed as diameters of other oligomers previously (Chimon et al. 2007, Ehrnhoefer et al. 2008). ThT emission, used to detect the cross- $\beta$ structure content in amyloid fibril, decreased down to $\sim 20 \%$ of the initial values after the incubation at -15 ${ }^{\circ} \mathrm{C}$ and there was no ThT fluorescence signal from the purified oligomers implying that the oligomers have no cross- $\beta$ structure (Fig. 6- $2 b$ ). 

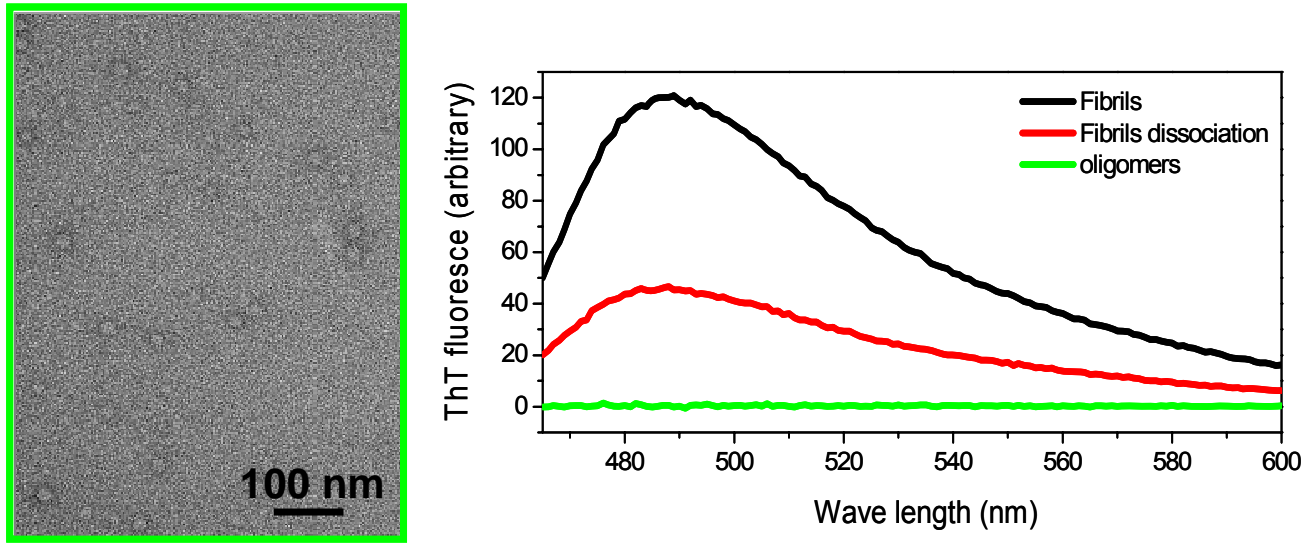

Fig. 6- 2. EM image and Thioflavin-T fluorescence of the oligomers.

EM image shows spherical morphology for the oligomers dissociated from amyloid fibril by super-cooled solution. The oligomers doesn't bind with ThT and give no fluoresce emission (green) while fibril and protofibril give rise ThT emission (black, ref).

The detailed images of the oligomers were obtained with AFM (Fig. 6- 3). The oligomers show a wide range of height from $3 \mathrm{~nm}$ to $10 \mathrm{~nm}$, and width from $20 \mathrm{~nm}$ to $100 \mathrm{~nm}$, which postulate heterogeneity of the oligomers.
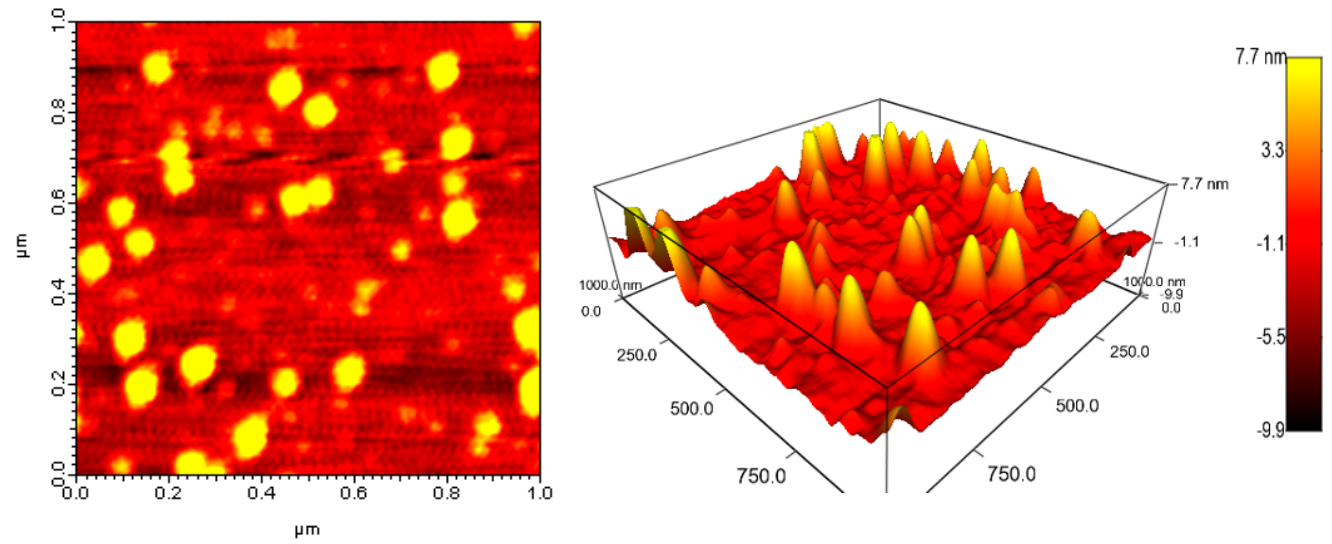

Fig. 6- 3. Atomic force microscopy image of the oligomers.

AFM image of the oligomers on glass surface in $1 \mu \mathrm{m} \times 1 \mu \mathrm{m}$ area is shown. Due to the scanning probe effect of AFM, the z-axis information is more reliable. 


\subsection{Summary and outlook}

To define the molecular dimension of the oligomers, we adopted microscopic method like EM and AFM, since the size hampers solution state NMR methods. Our preliminary observation revealed that the oligomers adopt spherical morphology with diameter of 15-20 nm or $20-30 \mathrm{~nm}$ from EM, 20 - $100 \mathrm{~nm}$ from AFM, which has been shown from other oligomers. The heterogeneous morphology of the oligomers is frequently observed in amyloidogenic proteins, and should be related to its toxicity and amyloid fibril formation without being degraded by cellular organelles. To address the relationship between the structure and the function of the oligomers, various spectroscopic methods including H/D exchange with NMR, solid-state NMR and EPR, and in vivo experiments with neurons are in progress. 


\section{Bibliography}

Anfinsen, C. B., Principles that govern the folding of protein chains, Science 1973, 181, 223-30.

Apetri, M. M., Maiti, N. C., Zagorski, M. G., Carey, P. R., Anderson, V. E., Secondary structure of alpha-synuclein oligomers: Characterization by raman and atomic force microscopy, J Mol Biol 2006, 355, 63-71.

Bai, Y., Milne, J. S., Mayne, L., Englander, S. W., Primary structure effects on peptide group hydrogen exchange, Proteins 1993, 17, 75-86.

Baldwin, R. L., A new perspective on unfolded proteins, Adv Protein Chem 2002, 62, $361-7$.

Baldwin, R. L., The search for folding intermediates and the mechanism of protein folding, Annu Rev Biophys 2008, 37, 1-21.

Battiste, J. L., Wagner, G., Utilization of site-directed spin labeling and high-resolution heteronuclear nuclear magnetic resonance for global fold determination of large proteins with limited nuclear overhauser effect data, Biochemistry 2000, 39, 5355-65.

Berg, J. M., Tymoczko, J. L., Stryer, L., Stryer, L., Biochemistry, 5th ed., W.H. Freeman, New York, 2002.

Berman, H. M., Westbrook, J., Feng, Z., Gilliland, G., Bhat, T. N., Weissig, H., Shindyalov, I. N., Bourne, P. E., The protein data bank, Nucleic Acids Res 2000, $28,235-42$.

Bernado, P., Bertoncini, C. W., Griesinger, C., Zweckstetter, M., Blackledge, M., Defining long-range order and local disorder in native alpha-synuclein using residual dipolar couplings, J Am Chem Soc 2005a, 127, 17968-9.

Bernado, P., Blanchard, L., Timmins, P., Marion, D., Ruigrok, R. W., Blackledge, M., A structural model for unfolded proteins from residual dipolar couplings and small-angle x-ray scattering, Proc Natl Acad Sci U S A 2005b, 102, 17002-7.

Bertoncini, C. W., Fernandez, C. O., Griesinger, C., Jovin, T. M., Zweckstetter, M., Familial mutants of alpha-synuclein with increased neurotoxicity have a 
destabilized conformation, J Biol Chem 2005a, 280, 30649-52.

Bertoncini, C. W., Jung, Y. S., Fernandez, C. O., Hoyer, W., Griesinger, C., Jovin, T. M., Zweckstetter, M., Release of long-range tertiary interactions potentiates aggregation of natively unstructured alpha-synuclein, Proc Natl Acad Sci U S A 2005b, 102, 1430-5.

Blackledge, M., Recent progress in the study of biomolecular structure and dynamics in solution from residual dipolar couplings, Progress in Nuclear Magnetic Resonance Spectroscopy 2005, 46, 23-61.

Bloch, F., Hansen, W. W., Packard, M., Nuclear induction, Physical Review 1946, 69, $127-$.

Bussell, R., Eliezer, D., Residual structure and dynamics in parkinson's diseaseassociated mutants of alpha-synuclein, The Journal of biological chemistry 2001, 276, 45996-6003.

Carulla, N., Caddy, G. L., Hall, D. R., Zurdo, J., Gairi, M., Feliz, M., Giralt, E., Robinson, C. V., Dobson, C. M., Molecular recycling within amyloid fibrils, Nature 2005, 436, 554-8.

Cavanagh, J., Protein nmr spectroscopy : Principles and practice, 2nd ed., Academic Press, Amsterdam ; Boston, 2007.

Chen, M., Margittai, M., Chen, J., Langen, R., Investigation of alpha-synuclein fibril structure by site-directed spin labeling, J Biol Chem 2007, 282, 24970-9.

Chimon, S., Shaibat, M. A., Jones, C. R., Calero, D. C., Aizezi, B., Ishii, Y., Evidence of fibril-like beta-sheet structures in a neurotoxic amyloid intermediate of alzheimer's beta-amyloid, Nat Struct Mol Biol 2007

Chiti, F., Dobson, C. M., Protein misfolding, functional amyloid, and human disease, Annu Rev Biochem 2006, 75, 333-66.

Cho, M. K., Kim, H. Y., Bernado, P., Fernandez, C. O., Blackledge, M., Zweckstetter, M., Amino acid bulkiness defines the local conformations and dynamics of natively unfolded alpha-synuclein and tau, J Am Chem Soc 2007, 129, 3032-3.

Creamer, T. P., Campbell, M. N., Determinants of the polyproline ii helix from modeling studies, Adv Protein Chem 2002, 62, 263-82.

Croke, R. L., Sallum, C. O., Watson, E., Watt, E. D., Alexandrescu, A. T., Hydrogen exchange of monomeric alpha-synuclein shows unfolded structure persists at 
physiological temperature and is independent of molecular crowding in escherichia coli, Protein Sci 2008, 17, 1434-45.

Del Mar, C., Greenbaum, E. A., Mayne, L., Englander, S. W., Woods, V. L., Jr., Structure and properties of alpha-synuclein and other amyloids determined at the amino acid level, Proc Natl Acad Sci U S A 2005, 102, 15477-82.

Delaglio, F., Grzesiek, S., Vuister, G. W., Zhu, G., Pfeifer, J., Bax, A., Nmrpipe: A multidimensional spectral processing system based on unix pipes, $J$ Biomol NMR 1995, 6, 277-93.

Dill, K. A., Chan, H. S., From levinthal to pathways to funnels, Nat Struct Biol 1997, 4, $10-9$.

Dill, K. A., Ozkan, S. B., Shell, M. S., Weikl, T. R., The protein folding problem, Annu Rev Biophys 2008, 37, 289-316.

Dobson, C. M., Protein folding and misfolding, Nature 2003, 426, 884-90.

Ehrnhoefer, D. E., Bieschke, J., Boeddrich, A., Herbst, M., Masino, L., Lurz, R., Engemann, S., Pastore, A., Wanker, E. E., Egcg redirects amyloidogenic polypeptides into unstructured, off-pathway oligomers, Nat Struct Mol Biol 2008, $15,558-66$.

Eisenberg, D., Nelson, R., Sawaya, M. R., Balbirnie, M., Sambashivan, S., Ivanova, M. I., Madsen, A. O., Riekel, C., The structural biology of protein aggregation diseases: Fundamental questions and some answers, Acc. Chem. Res. 2006, 39, $568-75$

Eliezer, D., Characterizing residual structure in disordered protein states using nuclear magnetic resonance, Methods Mol Biol 2007, 350, 49-67.

Englander, S. W., Hydrogen exchange and mass spectrometry: A historical perspective, J Am Soc Mass Spectrom 2006, 17, 1481-9.

Ernst, R. R., Nobel lecture. Nuclear magnetic resonance fourier transform spectroscopy, Biosci Rep 1992, 12, 143-87.

Fersht, A. R., From the first protein structures to our current knowledge of protein folding: Delights and scepticisms, Nat Rev Mol Cell Biol 2008, 9, 650-4.

Fiebig, K. M., Schwalbe, H., Buck, M., Smith, L. J., Dobson, C. M., Toward a description of the conformations of denatured states of proteins. Comparison of a random coil model with nmr measurements, J Phys Chem-Us 1996, 100, 2661- 
6.

Fink, A. L., Natively unfolded proteins, Curr Opin Struct Biol 2005, 15, 35-41.

Flory, P. J., Statistical mechanics of chain molecules, Hanser Publishers, Munich, 1969.

Gillespie, J. R., Shortle, D., Characterization of long-range structure in the denatured state of staphylococcal nuclease. I. Paramagnetic relaxation enhancement by nitroxide spin labels, J Mol Biol 1997, 268, 158-69.

Goddard, T. D., Kneller, D. G., Sparky 3, San Francisco, CA, University of California

Goedert, M., Alpha-synuclein and neurodegenerative diseases, Nat Rev Neurosci 2001, 2, 492-501.

Griffin, J. L., Metabonomics: Nmr spectroscopy and pattern recognition analysis of body fluids and tissues for characterisation of xenobiotic toxicity and disease diagnosis, Current opinion in chemical biology 2003, 7, 648-54.

Hardin, C., Pogorelov, T. V., Luthey-Schulten, Z., Ab initio protein structure prediction, Curr Opin Struct Biol 2002, 12, 176-81.

Heise, H., Hoyer, W., Becker, S., Andronesi, O. C., Riedel, D., Baldus, M., Molecularlevel secondary structure, polymorphism, and dynamics of full-length alphasynuclein fibrils studied by solid-state nmr, Proc Natl Acad Sci U S A 2005, 102, 15871-6.

Heise, H., Celej, M. S., Becker, S., Riedel, D., Pelah, A., Kumar, A., Jovin, T. M., Baldus, M., Solid-state nmr reveals structural differences between fibrils of wild-type and disease-related a53t mutant alpha-synuclein, J Mol Biol 2008, 380, 444-50.

Hoshino, M., Katou, H., Hagihara, Y., Hasegawa, K., Naiki, H., Goto, Y., Mapping the core of the beta(2)-microglobulin amyloid fibril by h/d exchange, Nat Struct Biol 2002, 9, 332-6.

Hoshino, M., Katou, H., Yamaguchi, K., Goto, Y., Dimethylsulfoxide-quenched hydrogen/deuterium exchange method to study amyloid fibril structure, Biochim Biophys Acta 2007, 1768, 1886-99.

Hoyer, W., Antony, T., Cherny, D., Heim, G., Jovin, T. M., Subramaniam, V., Dependence of alpha-synuclein aggregate morphology on solution conditions, $J$ Mol Biol 2002, 322, 383-93.

Jansen, R., Universität Dortmund (Dortmund), 2005. 
Jarymowycz, V. A., Stone, M. J., Fast time scale dynamics of protein backbones: Nmr relaxation methods, applications, and functional consequences, Chem Rev 2006, $106,1624-71$.

Jha, A. K., Colubri, A., Freed, K. F., Sosnick, T. R., Statistical coil model of the unfolded state: Resolving the reconciliation problem, Proc Natl Acad Sci U S A 2005, 102, 13099-104.

Jones, J. A., Wilkins, D. K., Smith, L. J., Dobson, C. M., Characterisation of protein unfolding by nmr diffusion measurements, Journal of Biomolecular NMR 1997, 10, 199-203.

Juan J. Zarranz, J. A., The new mutation, e46k, of ?-synuclein causes parkinson and lewy body dementia, Annals of Neurology 2004, 55, 164-73.

Karplus, M., Vicinal proton coupling in nuclear magnetic resonance, Journal of the American Chemical Society 1963, 85, 2870-\&.

Kay, L. E., Keifer, P., Saarinen, T., Pure absorption gradient enhanced heteronuclear single quantum correlation spectroscopy with improved sensitivity, Journal of the American Chemical Society 1992, 114, 10663-5.

Kaylor, J., Bodner, N., Edridge, S., Yamin, G., Hong, D. P., Fink, A. L., Characterization of oligomeric intermediates in alpha-synuclein fibrillation: Fret studies of y125w/y133f/y136f alpha-synuclein, J Mol Biol 2005, 353, 357-72.

Kempf, J. G., Loria, J. P., Protein dynamics from solution nmr: Theory and applications, Cell Biochem Biophys 2003, 37, 187-211.

Kheterpal, I., Zhou, S., Cook, K. D., Wetzel, R., Abeta amyloid fibrils possess a core structure highly resistant to hydrogen exchange, Proc Natl Acad Sci U S A 2000, 97, 13597-601.

Kheterpal, I., Wetzel, R., Hydrogen/deuterium exchange mass spectrometry--a window into amyloid structure, Acc Chem Res 2006, 39, 584-93.

Kim, H. Y., Cho, M. K., Riedel, D., Fernandez, C. O., Zweckstetter, M., Dissociation of amyloid fibrils of alpha-synuclein in supercooled water, Angew Chem Int Ed Engl 2008, 47, 5046-8.

Klein-Seetharaman, J., Oikawa, M., Grimshaw, S. B., Wirmer, J., Duchardt, E., Ueda, T., Imoto, T., Smith, L. J., Dobson, C. M., Schwalbe, H., Long-range interactions within a nonnative protein, Science 2002, 295, 1719-22. 
Kosen, P. A., Spin labeling of proteins, Methods Enzymol 1989, 177, 86-121.

Krishna, M. M., Hoang, L., Lin, Y., Englander, S. W., Hydrogen exchange methods to study protein folding, Methods 2004, 34, 51-64.

Kruger, R., Kuhn, W., Muller, T., Woitalla, D., Graeber, M., Kosel, S., Przuntek, H., Epplen, J. T., Schols, L., Riess, O., Ala30pro mutation in the gene encoding alpha-synuclein in parkinson's disease, Nat Genet 1998, 18, 106-8.

Kyte, J., Doolittle, R. F., A simple method for displaying the hydropathic character of a protein, J Mol Biol 1982, 157, 105-32.

Lakomek, N. A., Carlomagno, T., Becker, S., Griesinger, C., Meiler, J., A thorough dynamic interpretation of residual dipolar couplings in ubiquitin, Journal of Biomolecular Nmr 2006, 34, 101-15.

Lakomek, N. A., Walter, K. F. A., Fares, C., Lange, O. F., de Groot, B. L., Grubmuller, H., Bruschweiler, R., Munk, A., Becker, S., Meiler, J., Griesinger, C., Selfconsistent residual dipolar coupling based model-free analysis for the robust determination of nanosecond to microsecond protein dynamics, Journal of Biomolecular Nmr 2008, 41, 139-55.

Lashuel, H. A., Petre, B. M., Wall, J., Simon, M., Nowak, R. J., Walz, T., Lansbury, P. T., Alpha-synuclein, especially the parkinson's disease-associated mutants, forms pore-like annular and tubular protofibrils, Journal of molecular biology 2002, 322, 1089-102.

Lee, J. C., Langen, R., Hummel, P. A., Gray, H. B., Winkler, J. R., Alpha-synuclein structures from fluorescence energy-transfer kinetics: Implications for the role of the protein in parkinson's disease, Proc Natl Acad Sci U S A 2004, 101, 1646671.

Levinthal, C., in Mossbauer Spectroscopy in Biological Systems, Proceedings of a meeting held at allerton house, monticello, illinois. (Ed.: P. Debrunner, Tsibris, J. \& Munck, E.), University of Illinois Press, Urbana, Illinois, 1969, pp. 22-4.

Levitt, M. H., Spin dynamics : Basics of nuclear magnetic resonance, John Wiley \& Sons, Chichester; New York, 2001.

Liu, M., Mao, X.-a., Ye, C., Huang, H., Nicholson, J. K., Lindon, J. C., Improved watergate pulse sequences for solvent suppression in nmr spectroscopy, Journal of Magnetic Resonance 1998, 132, 125-9. 
Louhivuori, M., Paakkonen, K., Fredriksson, K., Permi, P., Lounila, J., Annila, A., On the origin of residual dipolar couplings from denatured proteins, J Am Chem Soc 2003, 125, 15647-50.

Luheshi, L. M., Crowther, D. C., Dobson, C. M., Protein misfolding and disease: From the test tube to the organism, Curr Opin Chem Biol 2008, 12, 25-31.

Luhrs, T., Ritter, C., Adrian, M., Riek-Loher, D., Bohrmann, B., Dobeli, H., Schubert, D., Riek, R., 3d structure of alzheimer's amyloid-beta(1-42) fibrils, Proc Natl Acad Sci U S A 2005, 102, 17342-7.

McEntyre, J., The encyclopedia of life sciences (www.Els.Net), Nature 2001, 413, 4589.

Mittag, T., Forman-Kay, J. D., Atomic-level characterization of disordered protein ensembles, Curr Opin Struct Biol 2007, 17, 3-14.

Mittermaier, A., Kay, L. E., New tools provide new insights in nmr studies of protein dynamics, Science 2006, 312, 224-8.

Mohana-Borges, R., Goto, N. K., Kroon, G. J., Dyson, H. J., Wright, P. E., Structural characterization of unfolded states of apomyoglobin using residual dipolar couplings, J Mol Biol 2004, 340, 1131-42.

Mori, S., Abeygunawardana, C., Johnson, M. O., Vanzijl, P. C. M., Improved sensitivity of hsqc spectra of exchanging protons at short interscan delays using a new fast hsqc (fhsqc) detection scheme that avoids water saturation, Journal of Magnetic Resonance, Series B 1995, 108, 94-8.

Mukrasch, M. D., Markwick, P., Biernat, J., Bergen, M., Bernado, P., Griesinger, C., Mandelkow, E., Zweckstetter, M., Blackledge, M., Highly populated turn conformations in natively unfolded tau protein identified from residual dipolar couplings and molecular simulation, J Am Chem Soc 2007, 129, 5235-43.

Ohkubo, Y. Z., Brooks, C. L., 3rd, Exploring flory's isolated-pair hypothesis: Statistical mechanics of helix-coil transitions in polyalanine and the c-peptide from rnase a, Proc Natl Acad Sci U S A 2003, 100, 13916-21.

Ohnishi, S., Lee, A. L., Edgell, M. H., Shortle, D., Direct demonstration of structural similarity between native and denatured eglin c, Biochemistry 2004, 43, 4064-70.

Ottiger, M., Delaglio, F., Bax, A., Measurement of $\mathrm{j}$ and dipolar couplings from simplified two-dimensional nmr spectra, Journal of Magnetic Resonance 1998, 
$131,373-8$.

Palmer, A. G., 3rd, Nmr characterization of the dynamics of biomacromolecules, Chem Rev 2004, 104, 3623-40.

Palmer, A. G., 3rd, Grey, M. J., Wang, C., Solution nmr spin relaxation methods for characterizing chemical exchange in high-molecular-weight systems, Methods Enzymol 2005, 394, 430-65.

Panchal, S. C., Bhavesh, N. S., Hosur, R. V., Improved 3d triple resonance experiments, hnn and $h n(c) n$, for $h n$ and $15 n$ sequential correlations in $(13 \mathrm{c}, 15 n)$ labeled proteins: Application to unfolded proteins, J Biomol NMR 2001, 20, 135-47.

Petkova, A. T., Ishii, Y., Balbach, J. J., Antzutkin, O. N., Leapman, R. D., Delaglio, F., Tycko, R., A structural model for alzheimer's beta -amyloid fibrils based on experimental constraints from solid state nmr, Proc. Natl. Acad. Sci. U.S.A. 2002, 99, 16742-7.

Piotto, M., Saudek, V., Sklenar, V., Gradient-tailored excitation for single-quantum nmr spectroscopy of aqueous solutions, Journal of biomolecular NMR 1992, 2, 661-5. Polymeropoulos, M. H., Lavedan, C., Leroy, E., Ide, S. E., Dehejia, A., Dutra, A., Pike, B., Root, H., Rubenstein, J., Boyer, R., Stenroos, E. S., Chandrasekharappa, S., Athanassiadou, A., Papapetropoulos, T., Johnson, W. G., Lazzarini, A. M., Duvoisin, R. C., Di Iorio, G., Golbe, L. I., Nussbaum, R. L., Mutation in the $\{$ alpha\}-synuclein gene identified in families with parkinson's disease, Science 1997, 276, 2045-7.

Prestegard, J. H., New techniques in structural nmr--anisotropic interactions, Nat Struct Biol 1998, 5 Suppl, 517-22.

Purcell, E. M., Torrey, H. C., Pound, R. V., Resonance absorption by nuclear magnetic moments in a solid, Physical Review 1946, 69, 37-8.

Radford, S. E., Dobson, C. M., From computer simulations to human disease: Emerging themes in protein folding, Cell 1999, 97, 291-8.

Ramachandran, G. N., Protein structure and crystallography, Science 1963, 141, 288-91.

Rose, G. D., Geselowitz, A. R., Lesser, G. J., Lee, R. H., Zehfus, M. H., Hydrophobicity of amino acid residues in globular proteins, Science 1985, 229, 834-8.

Rose, G. D., Unfolded proteins, Academic Press, Amsterdam ; Boston, 2002.

Ross, C. A., Poirier, M. A., Protein aggregation and neurodegenerative disease, Nature 
medicine 2004, 10 Suppl, S10-7.

Ruckert, M., Otting, G., Alignment of biological macromolecules in novel nonionic liquid crystalline media for nmr experiments, J Am Chem Soc 2000, 122, 7793-7.

Sattler, M., Schleucher, J., Griesinger, C., Heteronuclear multidimensional nmr experiments for the structure determination of proteins in solution employing pulsed field gradients, Progress in Nuclear Magnetic Resonance Spectroscopy 1999, 34, 93-158.

Schwalbe, H., Fiebig, K. M., Buck, M., Jones, J. A., Grimshaw, S. B., Spencer, A., Glaser, S. J., Smith, L. J., Dobson, C. M., Structural and dynamical properties of a denatured protein. Heteronuclear $3 \mathrm{~d} \mathrm{nmr}$ experiments and theoretical simulations of lysozyme in $8 \mathrm{~m}$ urea, Biochemistry 1997, 36, 8977-91.

Schwarzinger, S., Kroon, G. J., Foss, T. R., Wright, P. E., Dyson, H. J., Random coil chemical shifts in acidic $8 \mathrm{~m}$ urea: Implementation of random coil shift data in nmrview, J Biomol NMR 2000, 18, 43-8.

Schwarzinger, S., Kroon, G. J., Foss, T. R., Chung, J., Wright, P. E., Dyson, H. J., Sequence-dependent correction of random coil nmr chemical shifts, $J$ Am Chem Soc 2001, 123, 2970-8.

Schwarzinger, S., Wright, P. E., Dyson, H. J., Molecular hinges in protein folding: The urea-denatured state of apomyoglobin, Biochemistry 2002, 41, 12681-6.

Schweitzer-Stenner, R., Measey, T. J., The alanine-rich xao peptide adopts a heterogeneous population, including turn-like and polyproline ii conformations, Proc Natl Acad Sci U S A 2007, 104, 6649-54.

Segers-Nolten, I., van der Werf, K., van Raaij, M., Subramaniam, V., Quantitative characterization of protein nanostructures using atomic force microscopy, Conference proceedings : ... Annual International Conference of the IEEE Engineering in Medicine and Biology Society. IEEE Engineering in Medicine and Biology Society. Conference 2007, 1, 6608-11.

Shortle, D., Ackerman, M. S., Persistence of native-like topology in a denatured protein in $8 \mathrm{~m}$ urea, Science 2001, 293, 487-9.

Shults, C. W., Lewy bodies, Proc Natl Acad Sci U S A 2006, 103, 1661-8.

Siemer, A. B., Arnold, A. A., Ritter, C., Westfeld, T., Ernst, M., Riek, R., Meier, B. H., Observation of highly flexible residues in amyloid fibrils of the het-s prion, 
Journal of the American Chemical Society 2006, 128, 13224-8.

Sillen, A., Wieruszeski, J.-M., Leroy, A., Younes, A. B., Landrieu, I., Lippens, G., High-resolution magic angle spinning nmr of the neuronal tau protein integrated in alzheimer's-like paired helical fragments, Journal of the American Chemical Society 2005, 127, 10138-9.

Spillantini, M. G., Schmidt, M. L., Lee, V. M., Trojanowski, J. Q., Jakes, R., Goedert, M., Alpha-synuclein in lewy bodies, Nature 1997, 388, 839-40.

Stejskal, E. O., Tanner, J. E., Spin diffusion measurements - spin echoes in presence of a time-dependent field gradient, Journal of Chemical Physics 1965, 42, 288-\&.

Sung, Y. H., Eliezer, D., Secondary structure and dynamics of micelle bound beta- and gamma-synuclein, Protein Sci 2006, 15, 1162-74.

Tanford, C., Kawahara, K., Lapanje, S., Proteins in 6-m guanidine hydrochloride. Demonstration of random coil behavior, J Biol Chem 1966, 241, 1921-3.

Tjandra, N., Bax, A., Direct measurement of distances and angles in biomolecules by nmr in a dilute liquid crystalline medium, Science 1997, 278, 1111-4.

Tolman, J. R., Flanagan, J. M., Kennedy, M. A., Prestegard, J. H., Nuclear magnetic dipole interactions in field-oriented proteins: Information for structure determination in solution, Proc Natl Acad Sci U S A 1995, 92, 9279-83.

Tran, H. T., Pappu, R. V., Toward an accurate theoretical framework for describing ensembles for proteins under strongly denaturing conditions, Biophys. J. 2006, $91,1868-86$.

Udgaonkar, J. B., Multiple routes and structural heterogeneity in protein folding, Annu Rev Biophys 2008, 37, 489-510.

Ulmer, T. S., Bax, A., Cole, N. B., Nussbaum, R. L., Structure and dynamics of micellebound human alpha-synuclein, J Biol Chem 2005, 280, 9595-603.

Uversky, V. N., Lee, H. J., Li, J., Fink, A. L., Lee, S. J., Stabilization of partially folded conformation during alpha-synuclein oligomerization in both purified and cytosolic preparations, $J$ Biol Chem 2001a, 276, 43495-8.

Uversky, V. N., Li, J., Fink, A. L., Evidence for a partially folded intermediate in alphasynuclein fibril formation, J Biol Chem 2001b, 276, 10737-44.

Uversky, V. N., Neuropathology, biochemistry, and biophysics of alpha-synuclein aggregation, J Neurochem 2007, 103, 17-37. 
Uversky, V. N., Oldfield, C. J., Dunker, A. K., Intrinsically disordered proteins in human diseases: Introducing the $\mathrm{d} 2$ concept, Annu Rev Biophys 2008, 37, 215-46.

Vilar, M., Chou, H. T., Luhrs, T., Maji, S. K., Riek-Loher, D., Verel, R., Manning, G., Stahlberg, H., Riek, R., The fold of alpha-synuclein fibrils, Proc Natl Acad Sci U S A 2008, 105, 8637-42.

Whitmore, L., Wallace, B. A., Dichroweb, an online server for protein secondary structure analyses from circular dichroism spectroscopic data, Nucleic Acids Res 2004, 32, W668-73.

Wilkins, D. K., Grimshaw, S. B., Receveur, V., Dobson, C. M., Jones, J. A., Smith, L. J., Hydrodynamic radii of native and denatured proteins measured by pulse field gradient nmr techniques, Biochemistry 1999, 38, 16424-31.

Wishart, D. S., Sykes, B. D., Chemical shifts as a tool for structure determination, Methods Enzymol 1994, 239, 363-92.

Wishart, D. S., Bigam, C. G., Holm, A., Hodges, R. S., Sykes, B. D., 1h, 13c and 15n random coil $\mathrm{nmr}$ chemical shifts of the common amino acids. I. Investigations of nearest-neighbor effects, J Biomol NMR 1995, 5, 67-81.

Wu, K. P., Kim, S., Fela, D. A., Baum, J., Characterization of conformational and dynamic properties of natively unfolded human and mouse alpha-synuclein ensembles by nmr: Implication for aggregation, J Mol Biol 2008, 378, 1104-15.

Wuthrich, K., Nmr studies of structure and function of biological macromolecules (nobel lecture), Angew Chem Int Ed Engl 2003, 42, 3340-63.

Wüthrich, K., Nmr of proteins and nucleic acids, Wiley, New York, 1986.

Yang, D. W., Nagayama, K., A sensitivity-enhanced method for measuring heteronuclear long-range coupling constants from the displacement of signals in two 1d subspectra, Journal of Magnetic Resonance Series A 1996, 118, 117-21.

Zhang, Y., Progress and challenges in protein structure prediction, Curr Opin Struct Biol 2008, 18, 342-8.

Zimmerman, J. M., Eliezer, N., Simha, R., The characterization of amino acid sequences in proteins by statistical methods, $J$ Theor Biol 1968, 21, 170-201.

Zweckstetter, M., Bax, A., Prediction of sterically induced alignment in a dilute liquid crystalline phase: Aid to protein structure determination by nmr, J Am Chem Soc 2000, 122, 3791-2. 
Zweckstetter, M., Bax, A., Single-step determination of protein substructures using dipolar couplings: Aid to structural genomics, Journal of the American Chemical Society 2001, 123, 9490-1.

Zweckstetter, M., Nmr: Prediction of molecular alignment from structure using the pales software, Nat Protoc 2008, 3, 679-90. 


\section{Appendix}

\section{A.1. $w t \alpha \mathrm{S}$ backbone chemical shifts at pH3 in Na-acetate}

\begin{tabular}{|c|c|c|c|c|c|c|}
\hline$\#$ & $\mathrm{AA}$ & $\mathrm{HN}$ & $\mathrm{N}$ & $\mathrm{CA}$ & $\mathrm{CB}$ & $\mathrm{C}^{\prime}$ \\
\hline 1 & $\mathrm{M}$ & - & & 54.83 & 32.681 & \\
\hline 2 & $\mathrm{D}$ & 8.888 & 123.9 & 52.69 & 38.469 & 176.7 \\
\hline 3 & $\mathrm{~V}$ & 8.246 & 120.5 & 62.439 & 32.649 & 175.7 \\
\hline 4 & $\mathrm{~F}$ & 8.303 & 123.8 & 57.73 & 39.346 & 174.6 \\
\hline 5 & M & 8.186 & 122.5 & - & - & - \\
\hline 6 & $\mathrm{~K}$ & 8.268 & 123 & 56.525 & 32.747 & 176.5 \\
\hline 7 & G & 8.403 & 110.2 & 45.026 & - & . \\
\hline 8 & $\mathrm{~L}$ & 8.06 & 121.7 & 55.025 & 42.413 & 177.6 \\
\hline 9 & $\mathrm{~S}$ & 8.318 & 116.9 & 58.15 & 63.69 & 174.6 \\
\hline 10 & K & 8.393 & 123.7 & 56.54 & 32.685 & 176.6 \\
\hline 11 & A & 8.26 & 124.9 & 52.638 & 18.961 & 177.3 \\
\hline 12 & K & 8.271 & 120.7 & 56.351 & 32.832 & 176.7 \\
\hline 13 & E & 8.431 & 121.8 & 55.89 & 28.806 & 177.9 \\
\hline 14 & G & - & - & 45.313 & - & 174 \\
\hline 15 & V & 7.963 & 120.3 & 62.902 & 32.614 & 176.6 \\
\hline 16 & $\mathrm{~V}$ & 8.269 & 125.2 & 62.771 & 32.597 & \\
\hline 17 & A & 8.409 & 127.6 & - & - & 177.7 \\
\hline 18 & A & 8.28 & 123.5 & 52.616 & 18.946 & 177.9 \\
\hline 19 & A & 8.227 & 122.9 & 52.61 & 18.881 & 178.1 \\
\hline 20 & E & 8.229 & 119.4 & 55.887 & 28.666 & 176.4 \\
\hline 21 & K & 8.316 & 122.5 & 56.518 & 32.678 & 176.9 \\
\hline 22 & $\mathrm{~T}$ & 8.126 & 115.4 & 61.927 & 69.759 & 174.5 \\
\hline 23 & $\mathrm{~K}$ & 8.354 & 123.8 & 56.503 & 32.817 & 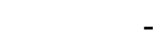 \\
\hline 24 & Q & - & - & 55.932 & 29.018 & 176.5 \\
\hline 25 & G & 8.438 & 110.5 & 45.297 & - & 174.2 \\
\hline 26 & V & 8.012 & 119.9 & 62.439 & 32.619 & 176.3 \\
\hline 27 & A & 8.395 & 128 & 52.565 & 18.903 & 177.8 \\
\hline 28 & E & 8.296 & 120.2 & 55.771 & 28.72 & 176.1 \\
\hline 29 & A & 8.303 & 125.3 & 52.518 & 18.965 & - \\
\hline 30 & A & - & - & 52.596 & 18.954 & 178.4 \\
\hline 31 & G & 8.314 & 108.4 & 45.255 & - & 174.2 \\
\hline 32 & $\mathrm{~K}$ & 8.154 & 120.8 & 56.109 & 33.018 & 177 \\
\hline 33 & $\mathrm{~T}$ & 8.19 & 115.6 & 61.945 & 69.762 & 174.5 \\
\hline 34 & $\mathrm{~K}$ & 8.44 & 124.1 & 56.276 & 32.832 & \\
\hline 35 & E & - & - & 55.89 & 28.806 & 176.5 \\
\hline 36 & $\mathrm{G}$ & - & - & 45.213 & - & 173.9 \\
\hline 37 & V & 7.963 & 119.5 & 62.387 & 32.645 & 176 \\
\hline 38 & $\mathrm{~L}$ & 8.279 & 125.9 & 54.589 & 42.409 & 176.6 \\
\hline 39 & Y & 8.266 & 122.6 & 57.773 & 38.704 & 175.6 \\
\hline 40 & V & 8.068 & 123.6 & 62.095 & 32.71 & 176.1 \\
\hline 41 & $\mathrm{G}$ & 8.027 & 112.2 & 45.089 & - & 173.9 \\
\hline 42 & $\mathrm{~S}$ & 8.244 & 115.6 & 58.168 & 63.81 & 174.9 \\
\hline 43 & K & 8.498 & 123.6 & 56.382 & 32.85 & 177 \\
\hline 44 & $\mathrm{~T}$ & 8.138 & 115.4 & 61.993 & 69.803 & 174.5 \\
\hline 45 & $\mathrm{~K}$ & 8.393 & 124 & 56.496 & 32.627 & 176.4 \\
\hline
\end{tabular}


Appendix

\begin{tabular}{|c|c|c|c|c|c|c|}
\hline 46 & $\mathrm{E}$ & 8.385 & 121.7 & 55.89 & 28.806 & - \\
\hline 47 & $\mathrm{G}$ & - & - & 45.269 & - & 173.8 \\
\hline 48 & $\mathrm{~V}$ & 7.981 & 119.9 & 62.223 & 32.607 & 176.1 \\
\hline 49 & $\mathrm{~V}$ & 8.303 & 125.1 & 62.139 & 32.66 & 175.9 \\
\hline 50 & $\mathrm{H}$ & 8.702 & 123.9 & 54.981 & 29.109 & 174.6 \\
\hline 51 & G & 8.479 & 110.8 & 45.074 & - & 173.7 \\
\hline 52 & $\mathrm{~V}$ & 8.118 & 119.7 & 61.927 & 32.922 & 176 \\
\hline 53 & A & 8.507 & 128.4 & 52.246 & 19.108 & 177.8 \\
\hline 54 & $\mathrm{~T}$ & 8.17 & 115.4 & 61.725 & 69.858 & 175.9 \\
\hline 55 & $\mathrm{~V}$ & 8.231 & 123.1 & 62.432 & 32.612 & 176.3 \\
\hline 56 & A & 8.417 & 127.8 & 52.448 & 18.954 & 178 \\
\hline 57 & $\mathrm{E}$ & 8.314 & 120 & 55.742 & 28.718 & 176.4 \\
\hline 58 & $\mathrm{~K}$ & 8.424 & 122.8 & 56.108 & 32.949 & 176.9 \\
\hline 59 & $\mathrm{~T}$ & 8.167 & 115.8 & 61.989 & 69.83 & 174.5 \\
\hline 60 & $\mathrm{~K}$ & 8.393 & 124.2 & 56.289 & 32.773 & 176.7 \\
\hline 61 & $\mathrm{E}$ & 8.386 & 121.8 & 55.628 & 28.583 & 175.3 \\
\hline 62 & Q & 8.371 & 120.9 & 56.038 & 29.19 & 175.8 \\
\hline 63 & $\mathrm{~V}$ & 8.262 & 122 & 61.974 & 32.689 & 176.3 \\
\hline 64 & $\mathrm{~T}$ & 8.294 & 118.2 & 61.723 & 69.819 & 174 \\
\hline 65 & $\mathrm{~N}$ & 8.526 & 122 & 52.943 & 38.842 & - \\
\hline 66 & $\mathrm{~V}$ & 8.262 & 121.3 & 62.63 & 32.47 & 176.8 \\
\hline 67 & G & 8.553 & 112.8 & 45.26 & - & 174.6 \\
\hline 68 & $\mathrm{G}$ & 8.219 & 109.3 & 44.991 & - & 173.7 \\
\hline 69 & A & 8.164 & 123.8 & 52.15 & 19.265 & 177.6 \\
\hline 70 & $\mathrm{~V}$ & 8.224 & 120.7 & - & - & 176.3 \\
\hline 71 & $\mathrm{~V}$ & 8.4 & 125.6 & 62.238 & 32.652 & 176.3 \\
\hline 72 & $\mathrm{~T}$ & 8.316 & 118.8 & 61.885 & 69.755 & 174.9 \\
\hline 73 & G & 8.424 & 111.8 & 45.18 & - & 174 \\
\hline 74 & $\mathrm{~V}$ & 8.085 & 119.6 & 61.901 & 32.66 & 176.6 \\
\hline 75 & $\mathrm{~T}$ & 8.301 & 119.1 & 62.018 & 69.762 & 174.1 \\
\hline 76 & A & 8.353 & 128.1 & 52.214 & 19.276 & 177.6 \\
\hline 77 & $\mathrm{~V}$ & 8.146 & 120.2 & 62.425 & 32.627 & 176 \\
\hline 78 & A & 8.414 & 128.2 & 52.426 & 18.939 & 177.7 \\
\hline 79 & Q & - & - & 55.632 & 29.573 & 176 \\
\hline 80 & $\mathrm{~K}$ & 8.427 & 123.2 & 56.183 & 33.031 & 176.7 \\
\hline 81 & $\mathrm{~T}$ & 8.258 & 116.8 & 61.884 & 69.836 & 174.4 \\
\hline 82 & $\mathrm{~V}$ & 8.305 & 123.4 & 62.296 & 32.669 & 176.1 \\
\hline 83 & $\mathrm{E}$ & 8.51 & 124.8 & 55.727 & 28.701 & 176.5 \\
\hline 84 & G & 8.498 & 110.8 & 45.121 & - & 174 \\
\hline 85 & A & 8.301 & 124.1 & 52.683 & 19.015 & 178.5 \\
\hline 86 & G & 8.492 & 108.8 & 45.226 & - & 174.3 \\
\hline 87 & $\mathrm{~S}$ & 8.14 & 115.8 & 58.22 & 63.826 & 174.7 \\
\hline 88 & I & 8.184 & 122.9 & 61.266 & 38.512 & 176.3 \\
\hline 89 & A & 8.342 & 128.1 & 52.489 & 18.928 & 177.6 \\
\hline 90 & A & - & - & 52.488 & 18.973 & - \\
\hline 91 & A & 8.269 & 123.4 & 52.564 & 18.946 & 178.1 \\
\hline 92 & $\mathrm{~T}$ & 8.085 & 112.7 & 61.931 & 69.772 & 175.1 \\
\hline 93 & $\mathrm{G}$ & 8.291 & 111 & 45.175 & - & 173.6 \\
\hline 94 & $\mathrm{~F}$ & 8.069 & 120.8 & 57.7 & 39.56 & 175.5 \\
\hline 95 & $\mathrm{~V}$ & 8.032 & 123.6 & 62.092 & 32.93 & 175.6 \\
\hline 96 & $\mathrm{~K}$ & 8.362 & 126.2 & 56.352 & 32.904 & 176.5 \\
\hline 97 & $\mathrm{~K}$ & 8.404 & 123.2 & 56.443 & 32.996 & 176.4 \\
\hline 98 & $\mathrm{D}$ & 8.493 & 120.1 & 53.016 & 38.093 & 176 \\
\hline 99 & Q & 8.358 & 121 & 55.859 & 29.161 & 174.6 \\
\hline 100 & $\mathrm{~L}$ & 8.241 & 123.1 & 55.208 & 42.267 & 177.9 \\
\hline 101 & G & 8.389 & 109.7 & 45.234 & - & 174.2 \\
\hline 102 & $\mathrm{~K}$ & 8.187 & 120.8 & 56.212 & 32.991 & 176.5 \\
\hline
\end{tabular}




\begin{tabular}{|c|c|c|c|c|c|c|}
\hline 103 & $\mathrm{~N}$ & 8.559 & 119.4 & 53.182 & 38.467 & 175.3 \\
\hline 104 & $\mathrm{E}$ & 8.329 & 120.6 & - & - & 175.9 \\
\hline 105 & $\mathrm{E}$ & 8.303 & 122.1 & 55.89 & 28.806 & 176.5 \\
\hline 106 & $\mathrm{G}$ & 8.424 & 110.5 & 44.892 & - & 173.3 \\
\hline 107 & $\mathrm{~A}$ & 8.11 & 124.9 & 50.491 & 18.022 & - \\
\hline 108 & $\mathrm{P}$ & - & - & 63.131 & 31.888 & 177 \\
\hline 109 & $\mathrm{Q}$ & 8.51 & 121.1 & 55.679 & 29.29 & 176 \\
\hline 110 & $\mathrm{E}$ & 8.418 & 121.6 & 55.89 & 28.806 & 176.4 \\
\hline 111 & $\mathrm{G}$ & 8.436 & 109.9 & 45.228 & - & 174 \\
\hline 112 & I & 7.956 & 119.8 & 61.199 & 38.516 & 176.4 \\
\hline 113 & $\mathrm{~L}$ & 8.308 & 125.7 & 55.117 & 41.993 & 174.9 \\
\hline 114 & $\mathrm{E}$ & 8.336 & 121.7 & 55.608 & 28.655 & 175.5 \\
\hline 115 & D & 8.377 & 119.5 & 52.606 & 37.978 & 174.7 \\
\hline 116 & $\mathrm{M}$ & 8.179 & 121.7 & 53.134 & 32.229 & 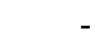 \\
\hline 117 & $\mathrm{P}$ & - & - & 62.913 & 32.041 & 175.2 \\
\hline 118 & V & 8.245 & 120.8 & 62.181 & 32.66 & 175.7 \\
\hline 119 & $\mathrm{D}$ & 8.587 & 123.7 & 50.673 & 38.229 & - \\
\hline 120 & $\mathrm{P}$ & - & - & 63.577 & 32.025 & 176.8 \\
\hline 121 & D & 8.324 & 117.6 & 52.962 & 37.835 & 175.1 \\
\hline 122 & $\mathrm{~N}$ & 8.168 & 118.8 & 53.166 & 38.489 & 175.3 \\
\hline 123 & $\mathrm{E}$ & 8.184 & 120.4 & 55.992 & 28.367 & 175.9 \\
\hline 124 & A & 8.217 & 124 & 52.676 & 18.85 & 177.4 \\
\hline 125 & $\mathrm{Y}$ & 7.935 & 118.5 & 57.667 & 38.526 & 175.4 \\
\hline 126 & $\mathrm{E}$ & 7.953 & 122 & 55.025 & 28.989 & 175.1 \\
\hline 127 & M & 8.235 & 122.7 & - & - & - \\
\hline 128 & $\mathrm{P}$ & - & - & 63.372 & 32.001 & 177.1 \\
\hline 129 & $\mathrm{~S}$ & 8.329 & 115.2 & 58.465 & 63.407 & 175.9 \\
\hline 130 & $\mathrm{E}$ & 8.423 & 122.2 & - & - & 176.1 \\
\hline 131 & $\mathrm{E}$ & 8.264 & 120.6 & 56.051 & 28.599 & 176.5 \\
\hline 132 & $\mathrm{G}$ & 8.344 & 109.8 & 45.189 & - & 174 \\
\hline 133 & Y & 8.003 & 120.2 & 58.03 & 38.486 & 176.6 \\
\hline 134 & Q & 8.342 & 121.6 & 55.528 & 29.388 & 175.1 \\
\hline 135 & $\mathrm{D}$ & 8.277 & 119.7 & 52.647 & 37.987 & 174.5 \\
\hline 136 & $\mathrm{Y}$ & 8.068 & 121 & 57.824 & 38.62 & 175.1 \\
\hline 137 & $\mathrm{E}$ & 8.054 & 123.9 & 52.718 & 28.601 & 20. \\
\hline 138 & $\mathrm{P}$ & - & - & 62.952 & 32.019 & 176.7 \\
\hline 139 & $\mathrm{E}$ & 8.388 & 120.9 & 55.559 & 28.788 & 175.7 \\
\hline 140 & A & 8.388 & 128.3 & 51.711 & 18.939 & - \\
\hline
\end{tabular}





\section{Lebenslauf}

\section{Persönliche Daten}

Name

Geburtstag

Geburtsort

Staatsangehörigkeit

\section{Ausbildung}

$10 / 2004 \sim 2008$

08/2004

07/2002 08/2004

03/1999 06/2001

02/1999

03/1995 02/1999

$03 / 1993 \sim 02 / 1995$
Min-Kyu Cho

23.11 .1976

Seoul, Süd Korea

Süd Korea

Doktorarbeit mit dem Titel "Transition of intrinsically unfolded $\alpha$-synuclein into the fibrillar state characterized by NMR spectroscopy" in der Abteilung NMR-basierte Strukturbiologie von Prof. Dr. Christian Griesinger und Dr. Markus Zweckstetter am Max Planck Institute für Biophysikalische Chemie in Göttingen

Master in Biochemie

Masterarbeit mit dem Titel "NMR Study on TA0743 and Exploring Protein Fold Space Using Data Distribution Method on Grid Platform" in der Gruppe von Prof. Dr. Weontae Lee an der Yonsei Universität in Seoul, Süd Korea

Wehrdienst

Bachelor in Chemie

Chemiestudium an der Seoul National Universität in Seoul, Süd Korea

Allgemeine Hochschulreife (Youido high school, Seoul, Süd Korea)

Göttingen, den 17. 9. 2008 